\title{
Minerva User Manual Version 1.0
}

\author{
J. J. Cogliati \\ M. L. Milvich \\ D. E. Wessol \\ C. A. Wemple
}

March 2007

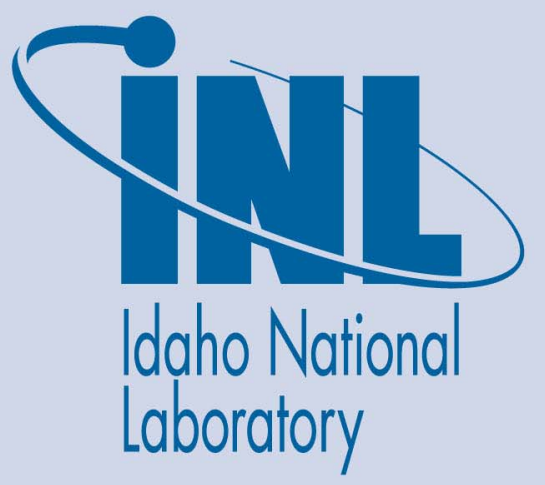

The INL is a U.S. Department of Energy National Laboratory operated by Battelle Energy Alliance 


\title{
Minerva User Manual Version 1.0
}

\author{
J. J. Cogliati \\ M. L. Milvich \\ D. E. Wessol \\ C. A. Wemple
}

March 2007

\section{Idaho National Laboratory \\ Idaho Falls, Idaho 83415}

Prepared for the

U.S. Department of Energy

Office of Science

Under DOE Idaho Operations Office

Contract DE-AC07-05ID14517 


\title{
Minerva User Manual Version 1.0
}

\author{
INL/EXT-07-12444
}

March 2007

Approved by

Vernon R Kubiak

$03 / 29 / 2007$

Type 1st persons name here

David W Nigg

Date

$03 / 28 / 2007$

Type 2nd persons name here

Date

Type 3rd persons name here

Date 


\section{SUMMARY}

MINERVA (Modality-Inclusive Environment for Radiotherapeutic Variable Analysis) is a Java-based patient-centric radiation treatment planning system (RTPS) for computational dosimetry and treatment planning in emerging areas of radiotherapy for cancer and other diseases. MINERVA was primarily developed at the Idaho National Laboratory (INL) and Montana State University (MSU). MINERVA allows the radiotherapist to make side-by-side comparison of plans for multiple treatment modalities with a common anatomical basis for the computational geometry, calculate doses for combinations of different radiotherapy modalities, and perform dose analysis and reporting functions. This provides the therapist with a consistent basis for selecting the modality or combination of modalities to use for treatment of the patient.

MINERVA employs an integrated, lightweight plug-in architecture to accommodate multi-modal treatment planning using standard interface components. The MINERVA design facilitates integration of improved or emerging treatment planning technologies.

MINERVA consists of the basic radiation treatment planning software modules managed by a consistent patient interface for developing multi-modal radiotherapy patient treatment plans. One of MINERVA's main functions is to provide a graphical environment for constructing and displaying uniform volume-element-based solid models derived from medical images. These solid models form the geometric basis of the target areas for the radiation transport model. 


\section{ACKNOWLEDGMENTS}

Thanks to past students and faculty at Montana State University including Clint Frederickson, Monique Perkins, Colt Frederickson, Gary Harkin, and Angela Kimmel for important contributions made in the design and programming of MINERVA. 


\section{CONTENTS}

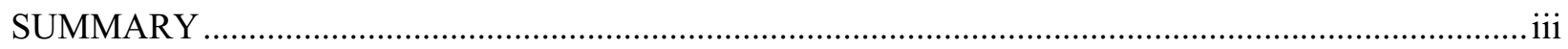

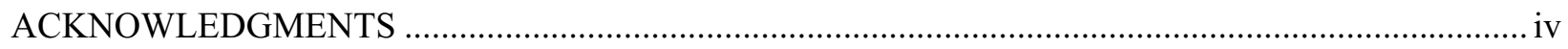

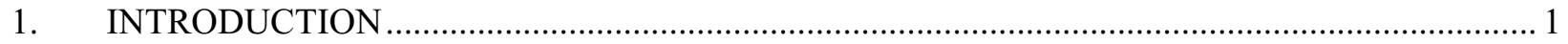

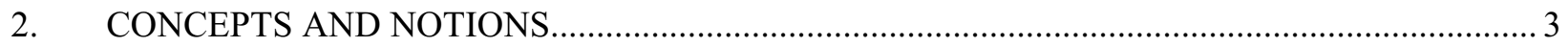

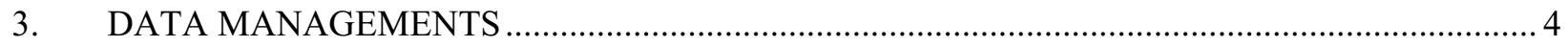

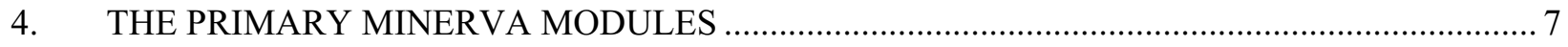

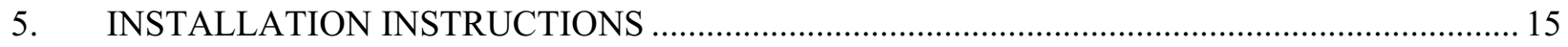

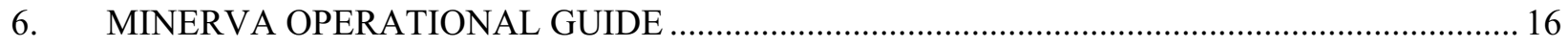

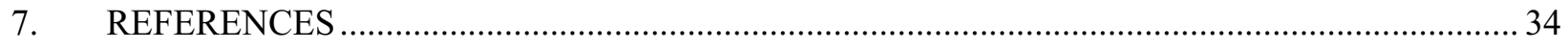

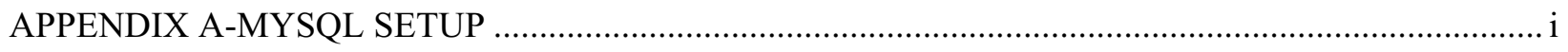

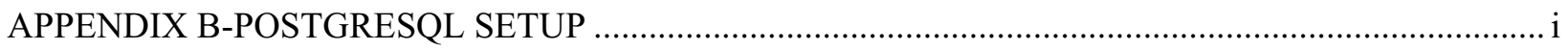

APPENDIX C-ADDITIONAL PACKAGES FOR INSTALLATION …............................................... i

APPENDIX D-XML FILE WITH DOSESET ….......................................................................

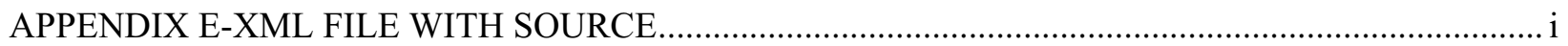




\section{FIGURES}

Figure 1. Basic design of the MINERVA treatment planning system. Dashed boxes represent future (to be developed) plug-in modules.

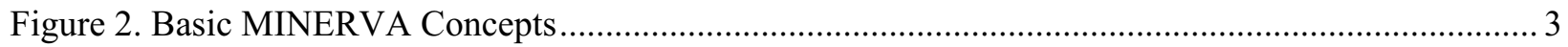

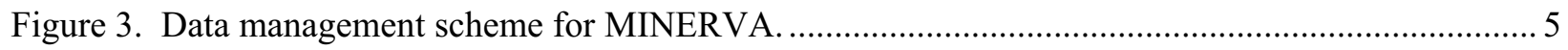

Figure 4. XML import/export menu in the Patient module. ................................................................ 6

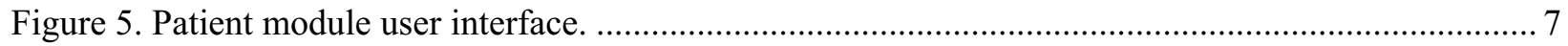

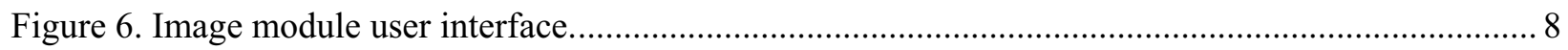

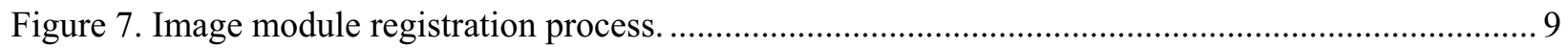

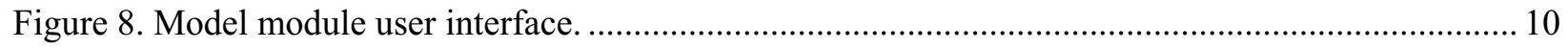

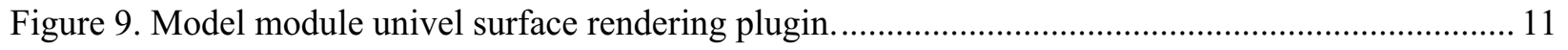

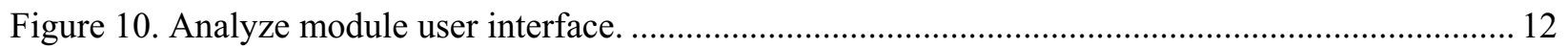

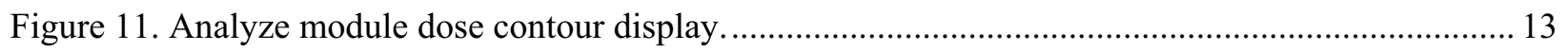

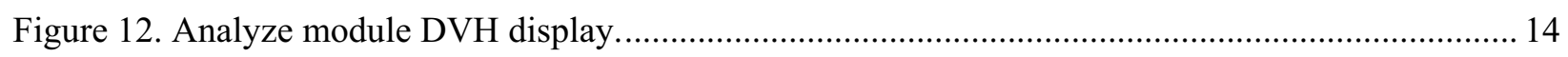

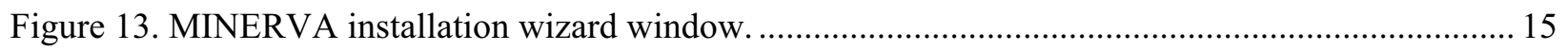

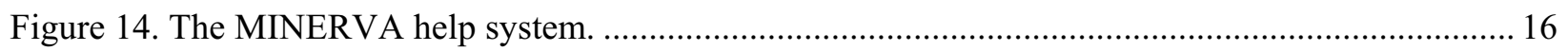

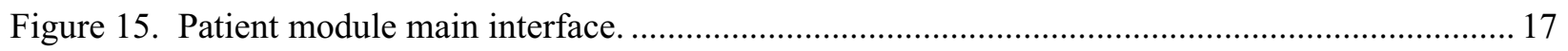

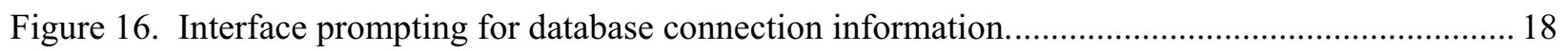

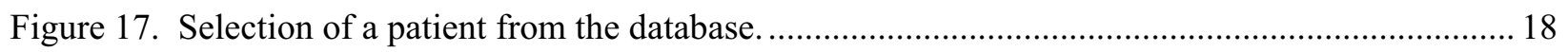

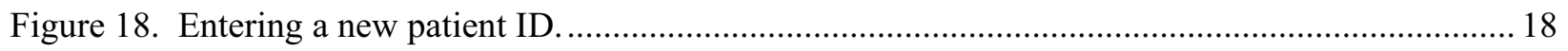

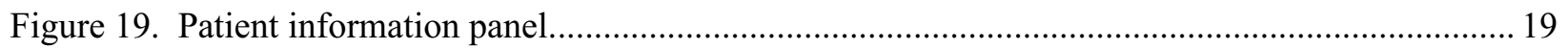

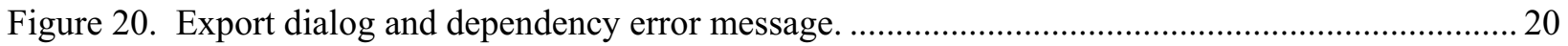

Figure 21. Display from the MINERVA Image module, showing co-registration of MR and PET images.21

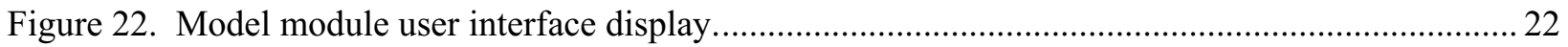

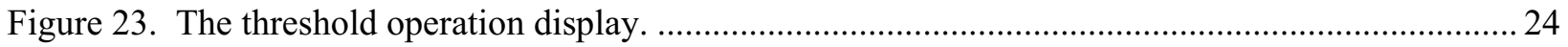

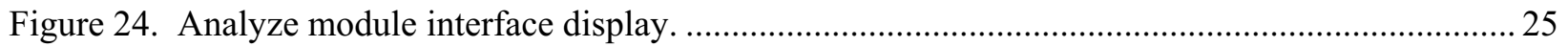




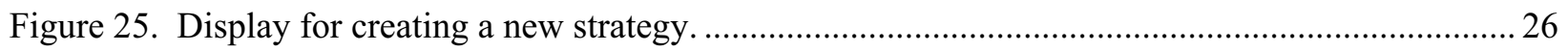

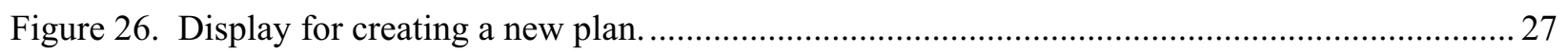

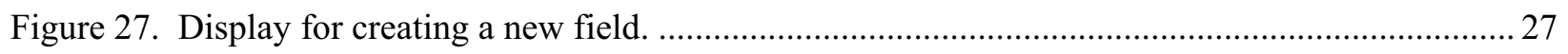

Figure 28. Analyze display showing a strategy with one plan and one field.......................................2 28

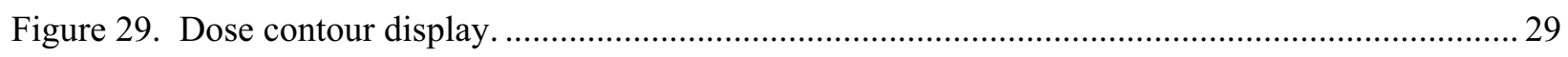

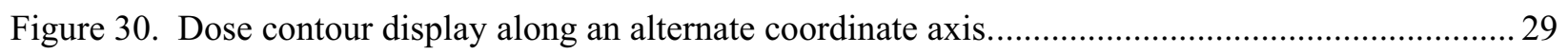

Figure 31. The Edit Plot Properties display for the contour plots..................................................... 30

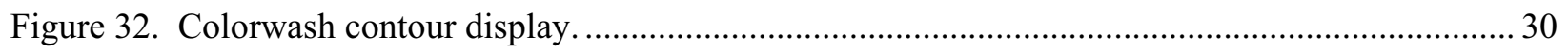

Figure 33. Colorwash contour display.Figure 33. Colorwash contour display..................................... 31

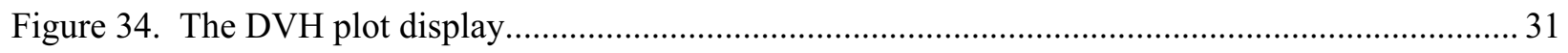

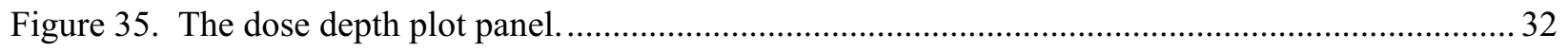

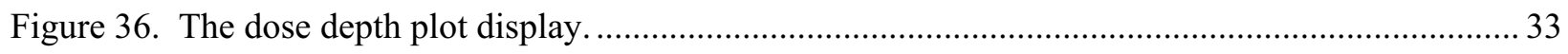




\section{INTRODUCTION}

MINERVA is a Java-based patient-centric radiation treatment planning system (RTPS) which can be used for planning and analyzing several radiotherapy modalities, either singly or combined, using common modality independent image and geometry construction and dose reporting and guiding. An example of combined planning would be an IMRT external beam treatment with a brachytherapy boost.

MINERVA is being developed with the Java Virtual Machine for interoperability. MINERVA employs an integrated, lightweight plug-in architecture to accommodate multi-modal treatment planning using standard interface components. The MINERVA design also facilitates the future integration of improved planning technologies.

As shown in the upper half of Figure 1, the MINERVA design includes the three common software modules used by any RTPS, along with a central patient module to coordinate communication and data transfer. The source and transport plug-in modules, shown on the left and bottom of Figure 1, communicate with these modules either directly through the patient database or through MINERVA's extensible markup language (XML)-based application programmer's interface (API). All internal data is managed by the database management system (DBMS) and can be exported to other applications or new installations through the API data formats.

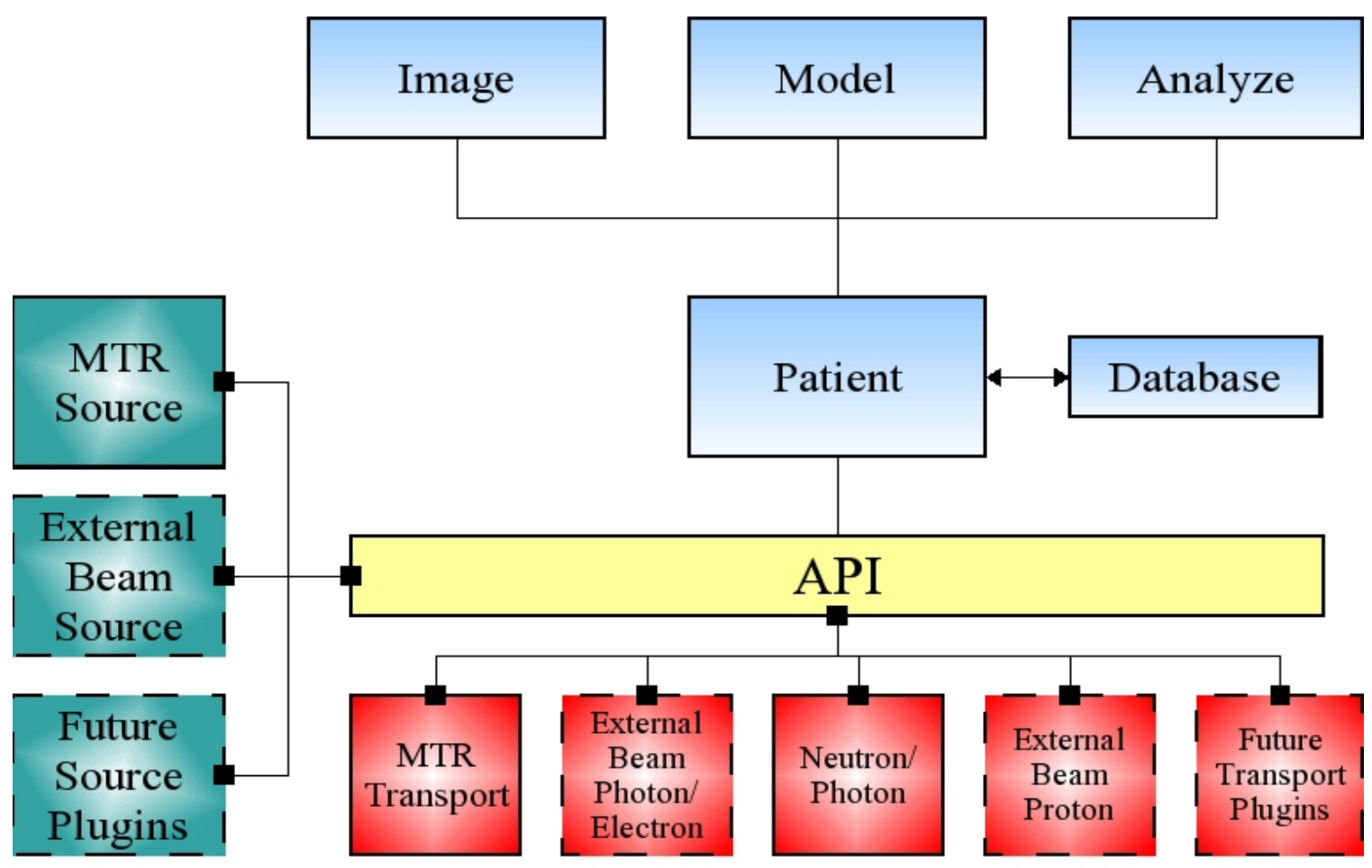

Figure 1. Basic design of the MINERVA treatment planning system. Dashed boxes represent future (to be developed) plug-in modules. 
In typical use, MINERVA consists of three main components:

- Manual and semi-automated geometric modeling of organs and physical structures derived from MRI, CT, and other medical imaging modalities;

- Dose computation for these geometric models with the appropriate radiation transport computer code;

- Dose reporting and combination to determine dose distribution and dose-volume histograms.

Digitized data produced from medical imaging devices are used as a template to draw, fill, paint, or automatically construct regions of interest in each image plane, forming a solid model description of the region of interest. These solid model descriptions are composed of rectangular parallelepipeds of uniform size, each uniquely mapped to a single organ or physical structure. The rectangular parallelepipeds are referred to as uniform volume elements (univels). Because of the uniformity of the univels, geometric interrogation can be performed using a very efficient integer arithmetic algorithm. Also, the resolution of the univels is only limited by the imaging modality, since each univel can be as small as an individual image pixel.

Using the solid model descriptions of the regions of interest, a three-dimensional radiation transport calculation determines the radiation fields present for a particular treatment modality. These radiation fields are then related to radiation doses delivered to the treatment volume and surrounding areas.

When the planning calculations are complete, the radiation fields are displayed either as dose contours in the original image space, dose volume histograms, or dose-depth plots. This gives the clinician or researcher an accurate and convenient way to view the radiation fields, so that the modality and dose delivery parameters can be quickly determined, balancing tumor cell kill against healthy tissue sparing.

The remainder of this manual provides a guide to the practical use of MINERVA's basic modules, but is not an exhaustive treatment of each feature of the code. 


\section{CONCEPTS AND NOTIONS}

The basic concepts used by MINERVA are image slices and regions, as illustrated in Figure 2. An image slice is data corresponding to a single medical image. Typically, several anatomical features of interest will intersect an image slice. For example, an image slice can contain a cranial object, tumor object, and objects defining eyes, sinuses, and other relevant features.

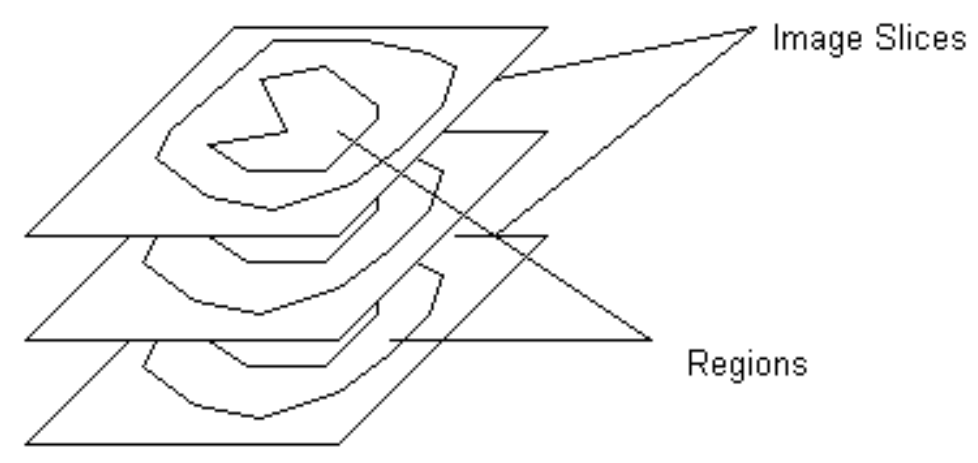

Figure 2. Basic MINERVA Concepts

Regions are defined by segmenting the data in a set of image slices. Once defined, region definitions in different slices are combined to form three-dimensional volumes.

Typically, the user begins by displaying an image slice, and then defining regions on that image. After several regions have been defined, they may be reconstructed (or synthesized) into a three dimensional object representing a structural or functional feature. These reconstructions can be displayed in a threedimensional viewing window, where viewing controls allow arbitrary views of the reconstructed data.

The univel, or Uniform Volume Element, geometry (Frandsen 1998, Frandsen 2001) is fundamental to the MINERVA geometry description. A univel is essentially a three-dimensional image pixel with uniform slice spacing between image slices. This representation gives significant computational advantages for Monte Carlo transport dose calculation and simplifies the description and display of the data. Note that the image data used for segmentation must have a uniform spacing between slices; if imported image data does not have uniform slice spacing; tools are provided to re-slice the image data to achieve this uniform spacing. 


\section{DATA MANAGEMENTS}

MINERVA's data management system employs an interface layer to provide a framework for modules to communicate with the data sources. As a result, the data sources are loosely coupled to the rest of the MINERVA. This allows new data storage technologies to be added without cascading effects throughout the system. Although not every module accesses all of the data sources, the data interface layer gives them the ability to do so, if needed.

Currently, MINERVA supports two types of data storage, relational databases and XML files. When MINERVA is first invoked, the Patient module will create the data interface layer, which can be utilized by the Image, Model, and Analyze modules. When data is required from a data source, a module issues a request to the data interface layer with a unique data identifier and the data source to examine. Once the data is retrieved, it will be cached in memory or on disk if memory availability is low. Subsequent requests for that data will retrieve the data out of the fastest cache available, thus improving the system's response time.

\subsection{Database}

All patient data is stored in a relational database, removing the burden of tracking multiple patient files from the user. The Java Database Connectivity (JDBC) technology allows MINERVA to communicate with almost any database that has a JDBC driver available for it.

The Patient, Image, Model, and Analyze modules have working database access. The database connection is established as part of the data interface layer setup by the Patient module.

Steps have been taken to ensure that the database integrity is maintained by using database transactions and foreign key constraints. Database transactions allow MINERVA to revert to a previous state if there was an error while saving an object, keeping the database from becoming corrupted. The foreign key constraints prevent MINERVA from removing items from the database until all references to the item have been removed, preventing accidental partial deletion of items.

\subsection{File Import/Export Features}

Because database files are generally hidden from the user and not easily transportable, a convenient way to transfer patient information between various users and installations was developed. To accomplish this, a complete Extensible Markup Language (XML) file specification of all of the information contained in the database and a set of XML-based import/export tools are being developed. This same XML file specification will also be used to help define the API between MINERVA and the various source and transport plugin modules. 


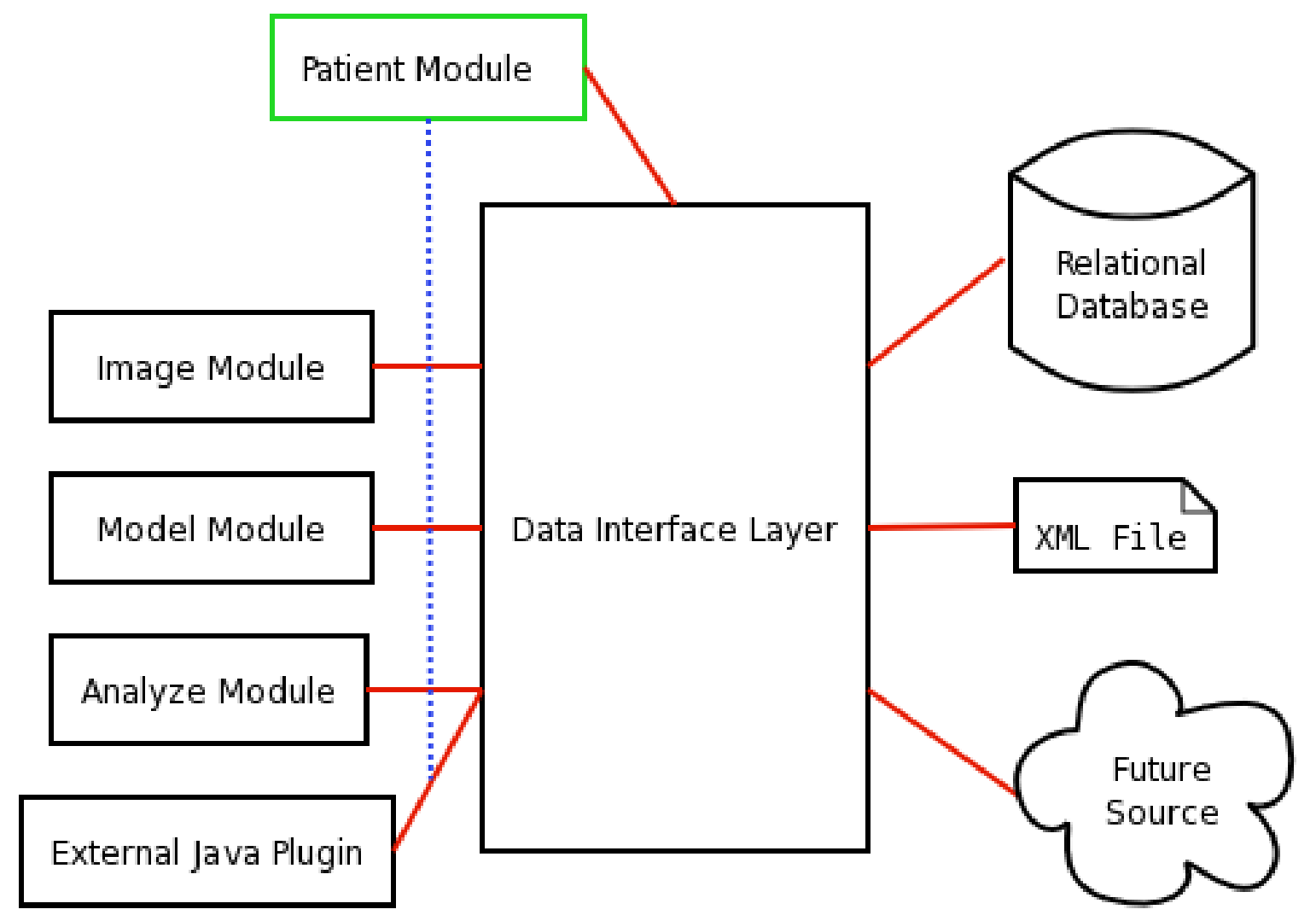

Figure 3. Data management scheme for MINERVA.

The XML-based tools allow any module that has access to the data interface layer to perform the following functions:

1. import external patient XML files directly into the database;

2. import XML files generated by an external plugin;

3. export specific data needed by an external plugin to perform an operation;

4. export all or part of a patient's data.

Tools 1 and 4 can be used to transport a patient or partial patient from one installation to another, or to backup a patient's data in a form not specific to any database vendor. Tools 2 and 3 allow external plugins to acquire information from, and provide information to, the MINERVA system.

The user can directly access the XML import/export features from the Patient module's toolbar, as shown in Figure 4. The menu options activate or deactivate situationally based on previous user actions. The user may export an entire patient, or export only the data of interest. The import command may import an entire XML document into the database, or only import a subsection of the XML document. Dependency checking will be done to maintain the integrity of the database. Other modules have access to XML functionality, should they need to use it. 


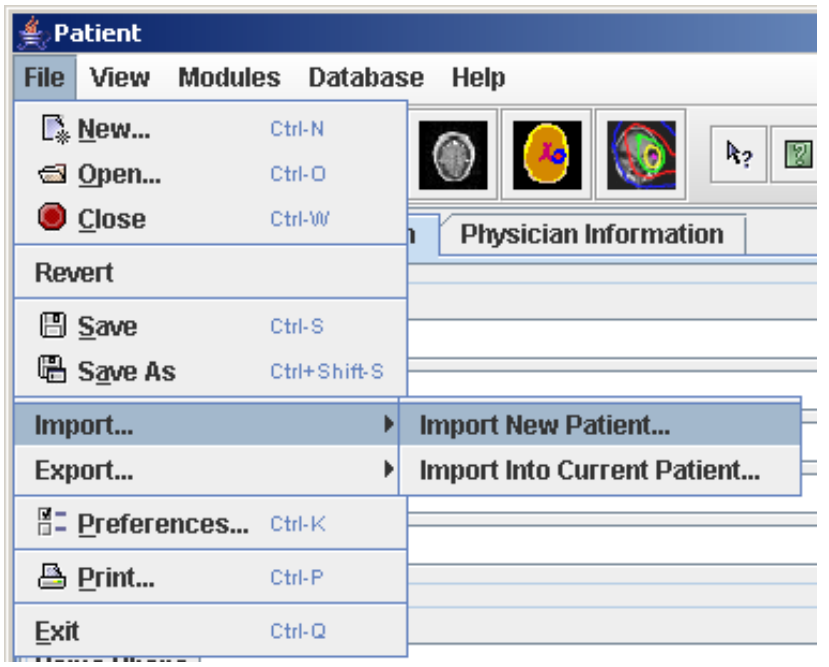

Figure 4. XML import/export menu in the Patient module. 


\section{THE PRIMARY MINERVA MODULES}

The Patient, Image, Model, and Analyze modules of the MINERVA system are summarized in the following sections.

\subsection{Patient Module}

The Patient module, which is the entry point to, and the hub of, the MINERVA system, manages the entire treatment planning process. In addition to launching and managing the Image, Model, and Analyze modules, the Patient module creates, edits, and retrieves data from the patient database.

The Patient module user interface allows the user to record, review, and edit patient information. A screen shot of the user interface is shown in Figure 5. This information includes some standard fields (name, address, phone, e-mail, date of birth, gender, etc.), but also includes user-specified fields. The Patient module allows the user to see what other data is associated with a given patient.

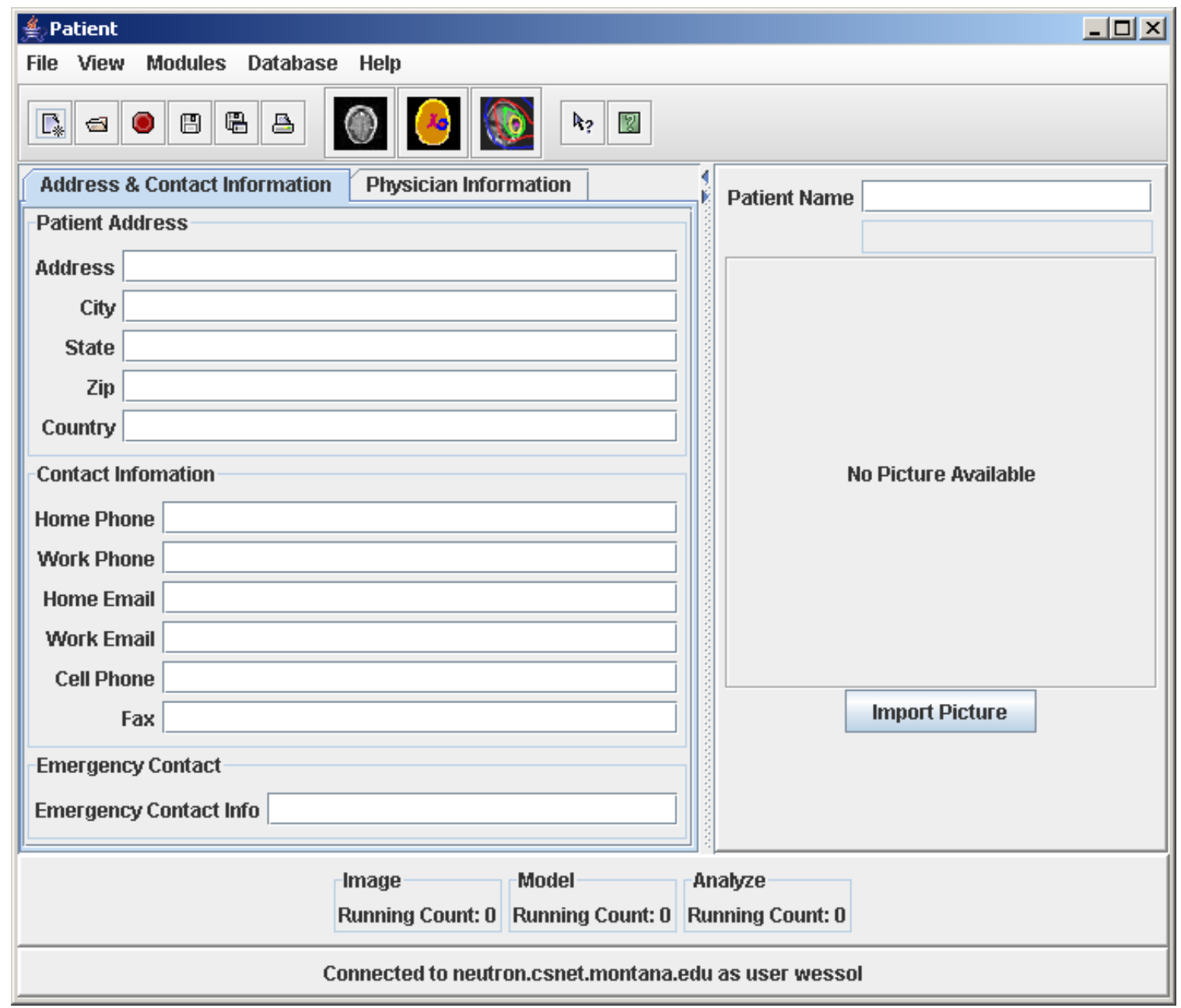

Figure 5. Patient module user interface. 
The Patient module controls MINERVA by launching the other modules, managing memory and data, allowing the user to view and modify preferences for the software's behavior and appearance, and maintaining a list of the most recently used patient data.

The Patient module is able to open a robust connection to the patient database using a Java Database Connectivity (JDBC) compliant database engine. Thus far, it has been tested using the MySQL (Widenius and Axmark 2002), PostgreSQL, and Derby database systems. A complete user interface is provided so that the planner can easily create, edit, or retrieve the patient data. If the Patient module is unable to complete the database connection, the program can still run and provide diagnostics to the user.

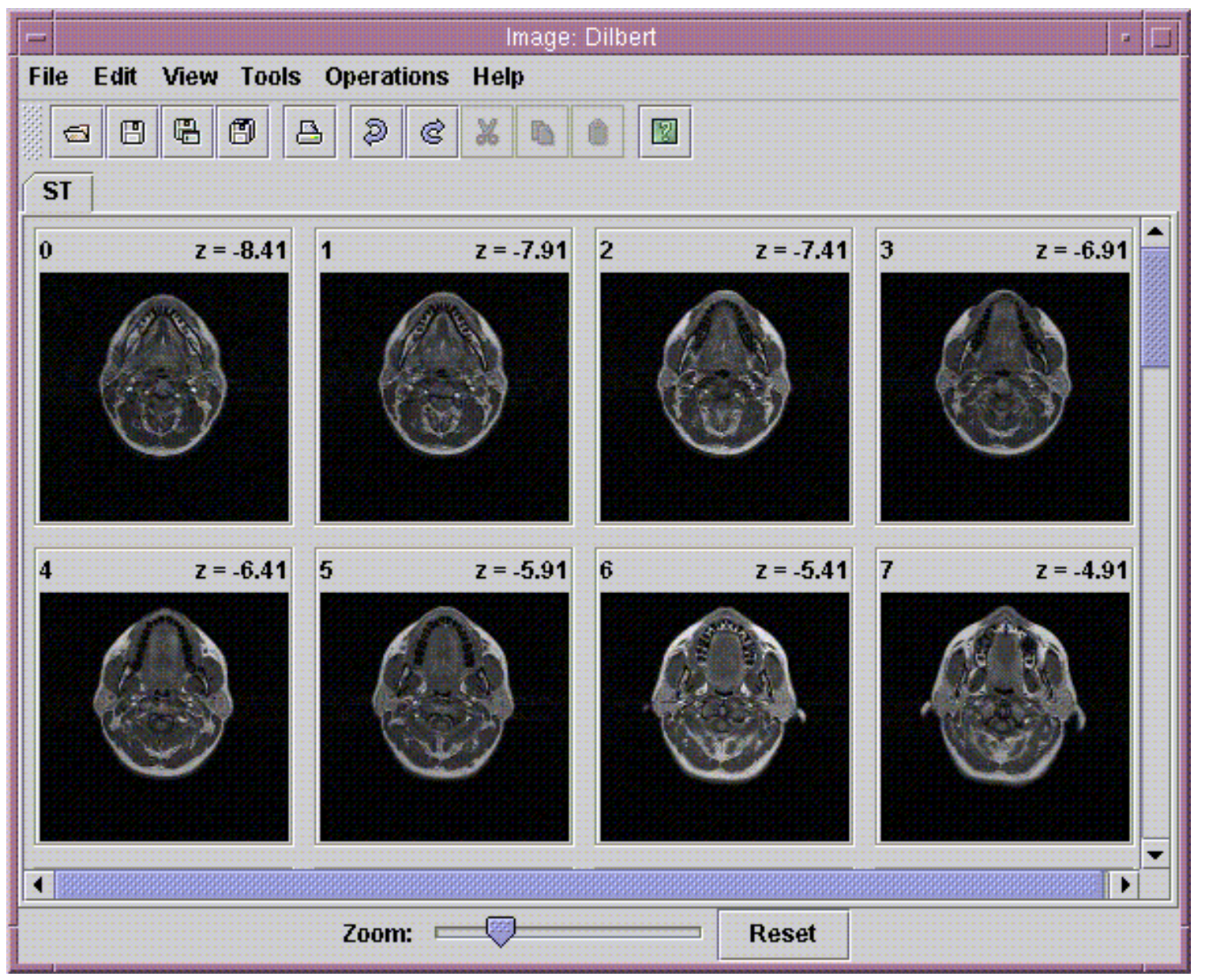

Figure 6. Image module user interface.

Patient information may be imported to, or exported from, the patient database using the XML-based API data formats.

\subsection{Image Module}

The Image module prepares and processes individual images and complete image sets for use by subsequent analysis modules. 
The user interface for the Image module is shown in Figure 6. Full database connectivity is included in the Image module. The Image module currently supports QSH, grayscale JPEG, grayscale PNG, and raw image import functions and partially supports DICOM-RT. With appropriate plugins, the Image module can convert other image formats to the internal MINERVA image format and store this data in the patient database. Plugin support allows additional image manipulation operations and import filters to be added at a later date. Standard image processing features, including noise filtering, gamma correction, and contrast enhancement, are provided.

A rigid body registration system based on maximization of mutual information (Viola and Wells 1997) has been included, with a three-dimensional display of the image registration process (see Figure 7). To facilitate the registration and define the geometry, a reslicing tool has been developed. This tool performs a pixel-by-pixel interpolation between existing image slices to define new images and create a uniform spacing between image slices, if one does not already exist in the original image set.

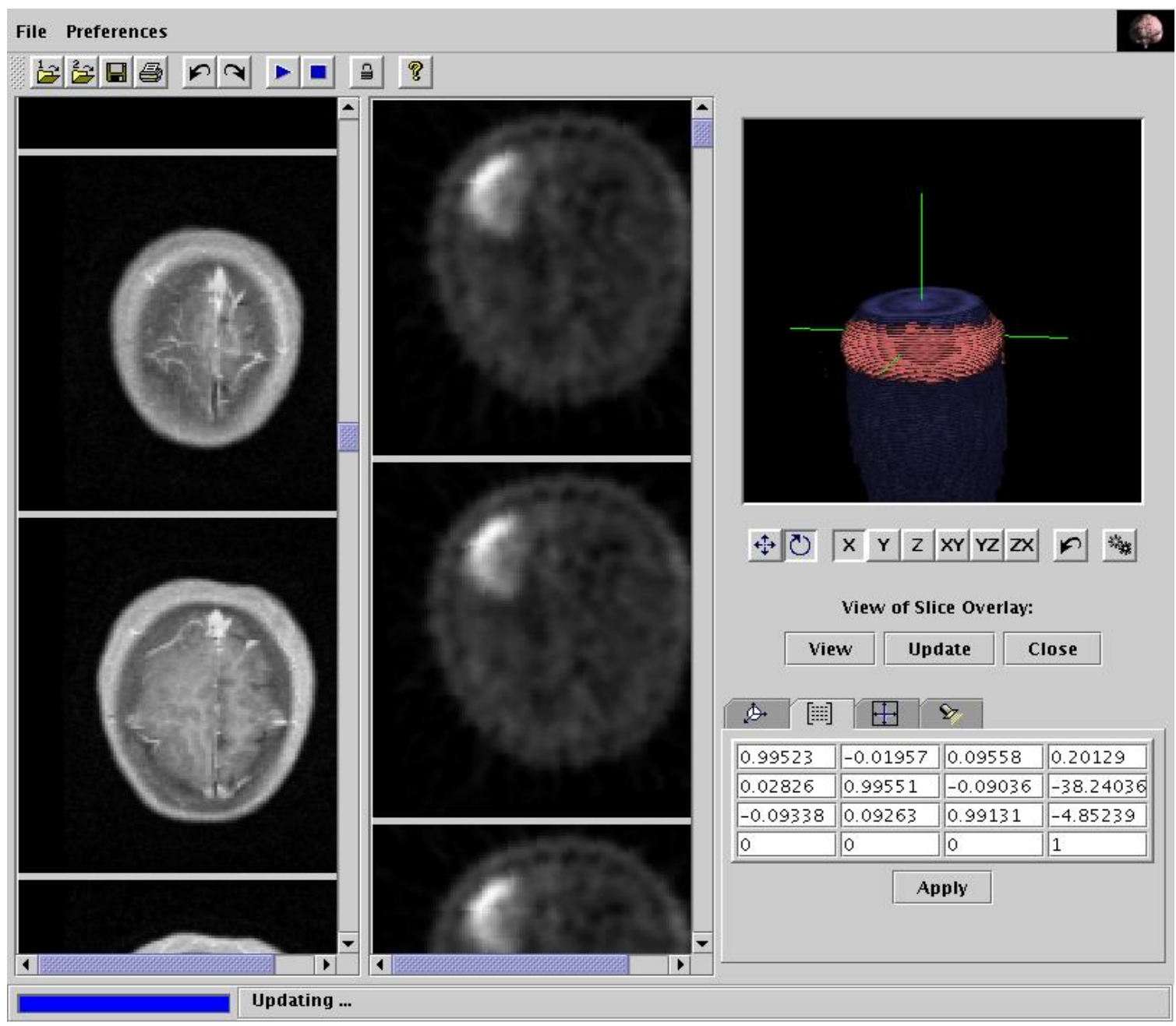

Figure 7. Image module registration process. 


\subsection{Model Module}

The Model module creates the anatomical geometry of the patient and target based on the image data received from the Image module. The Model module can be used to either create a new univel geometry file or edit an existing model. Anatomical structures are defined by various methods, and materials are assigned to these regions. The univel geometry permits very fast Monte Carlo analysis. The user interface and some of the features of the Model module are shown in Figure 8.

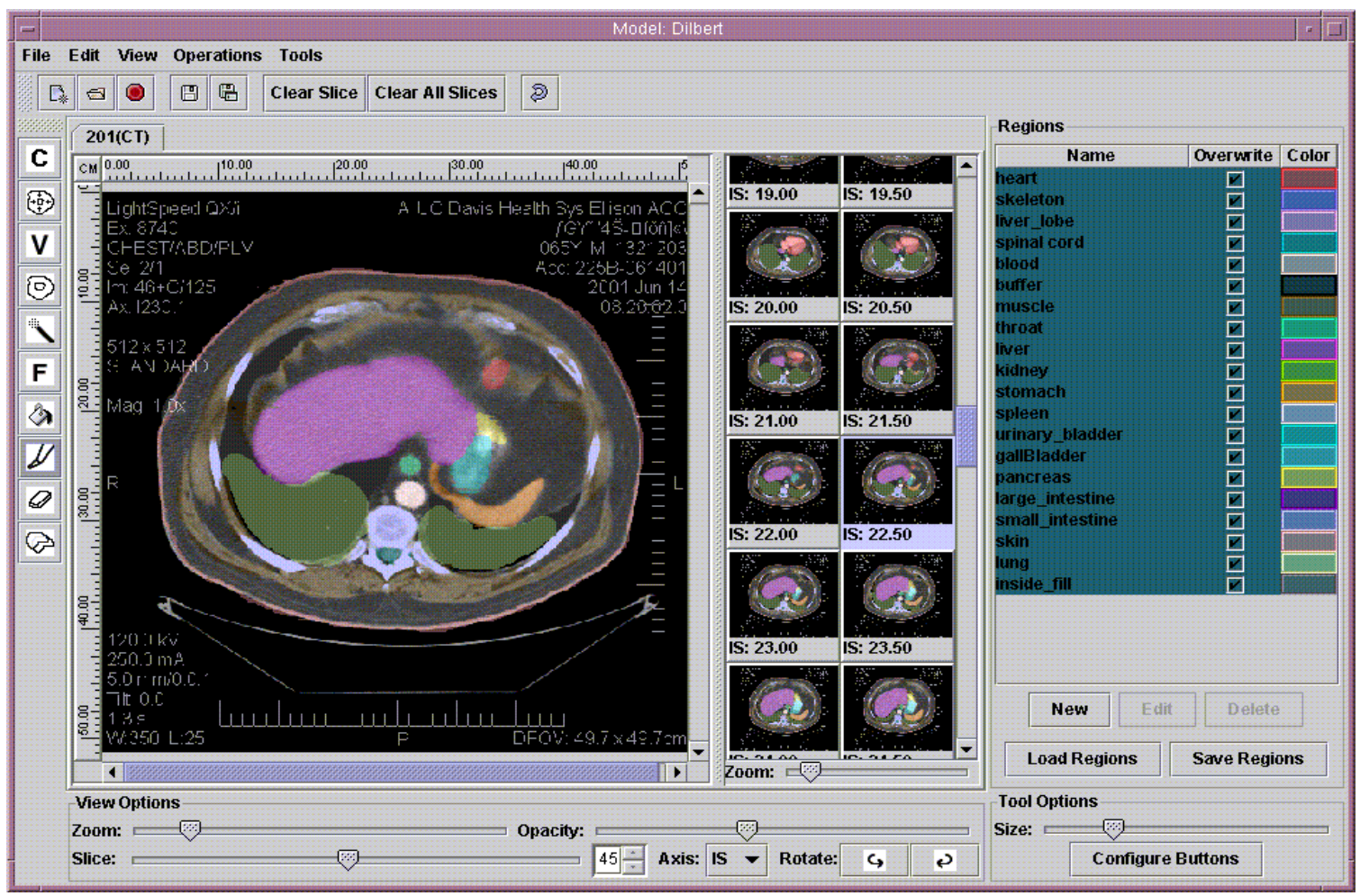

Figure 8. Model module user interface.

The Model module contains several methods for manually or semi-automatically defining up to 32,768 regions of interest (ROIs) from one or more image sets, which may encompass several imaging modalities. A great deal of effort was expended to make manual ROI definition easy and fast, as an interim solution until a suitable and robust automated procedure can be developed. All of the tools and operations have been implemented as plugins to simplify the program design and to allow the later addition of more advanced tools. A generic undo manager will record the user's actions, and allow them to be reversed. All modeling can be conducted with multiple image sets along the three principal orthogonal axes simultaneously and interchangeably. Multiple image sets may be used simultaneously to define the ROIs.

Zooming support is included, and the display of image slices is optimized for speed and memory usage. A 3D visualization view, shown in Figure 9, can be used to display the current geometry and will update as the user constructs the geometry to give interactive feedback. 

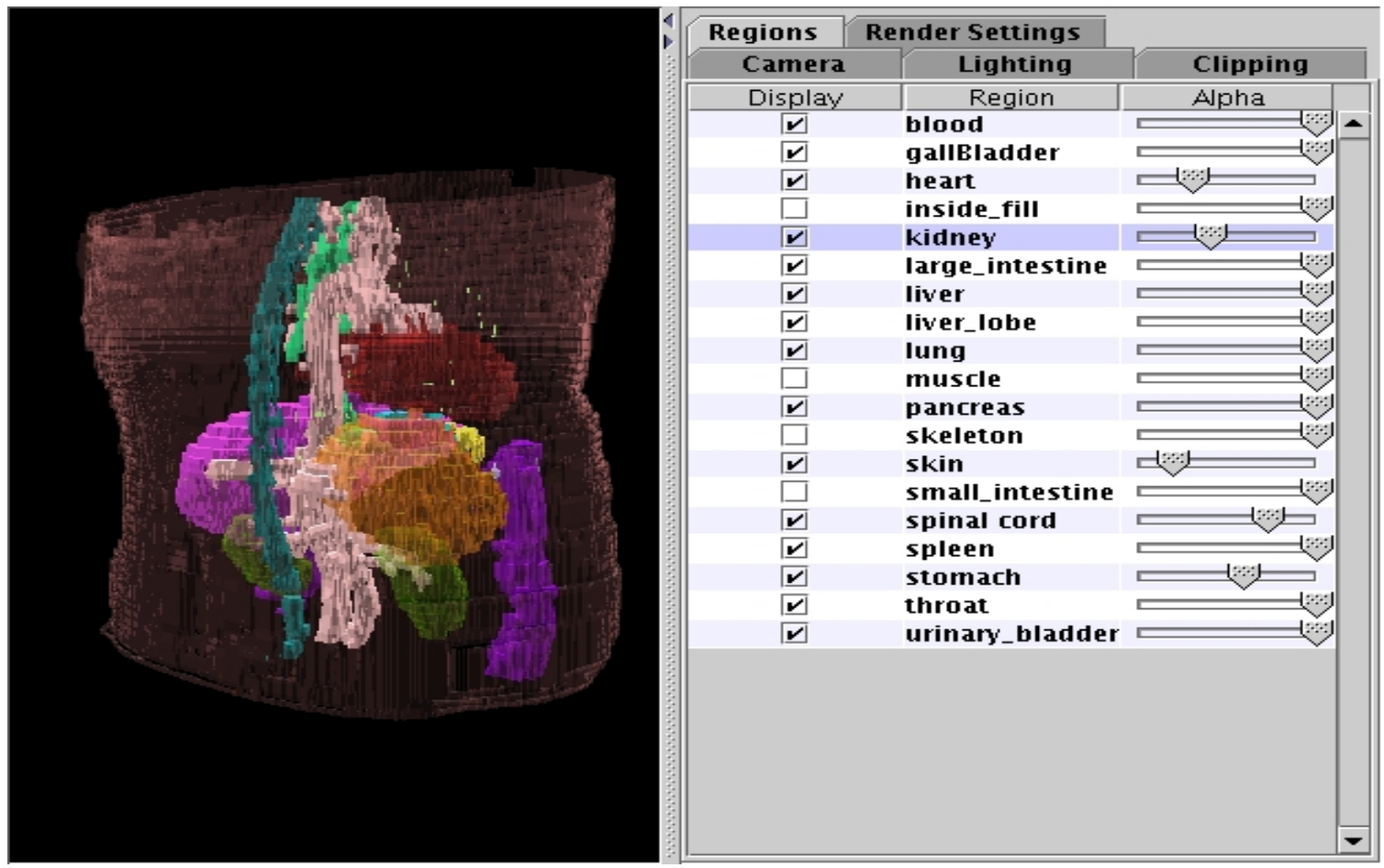

Figure 9. Model module univel surface rendering plugin.

The Model module also supports scripting, where significant user actions are recorded to a file that can be played back later. Either an entire or partial modeling session can be recorded, giving the user the ability to automate common tasks. With this feature, modeling sessions can be replicated easily or stored as planning templates. This has the capability of validating and simplifying the modeling process.

\subsection{Analyze Module}

Control of plan development and dose reporting is consolidated in the Analyze module. The planning function controls the launching of the source and transport plugins, receives the results from these plugin modules, and saves the results to the database. The dose reporting function accesses the database to read the dose information for each field in the plan, and performs the dose combination and reporting. An example of the Analyze module capabilities is shown in Figure 10.

The Analyze module planning control, shown in the upper (tabbed) section of Figure 10, can create multiple strategies, plans, and fields, and can combine fields into plans and plans into strategies. For the MINERVA system, a field is defined as a single radiation application, a plan is a single radiation therapy modality application, and a strategy is a set of plans administered to a patient. Basic checks are executed before inserting a field or a plan, such as checking the dimensions and modality types to assure consistency. The Analyze module launches source plugins to create the radiation source descriptions for each field, and transport plugins to calculate the dose distribution. (The dose data are included in the MINERVA field definition.) A default transport plugin was created to export the field information, with its associated source and geometry, to an XML file and import calculated dose data from an XML file and associate it with the field. 


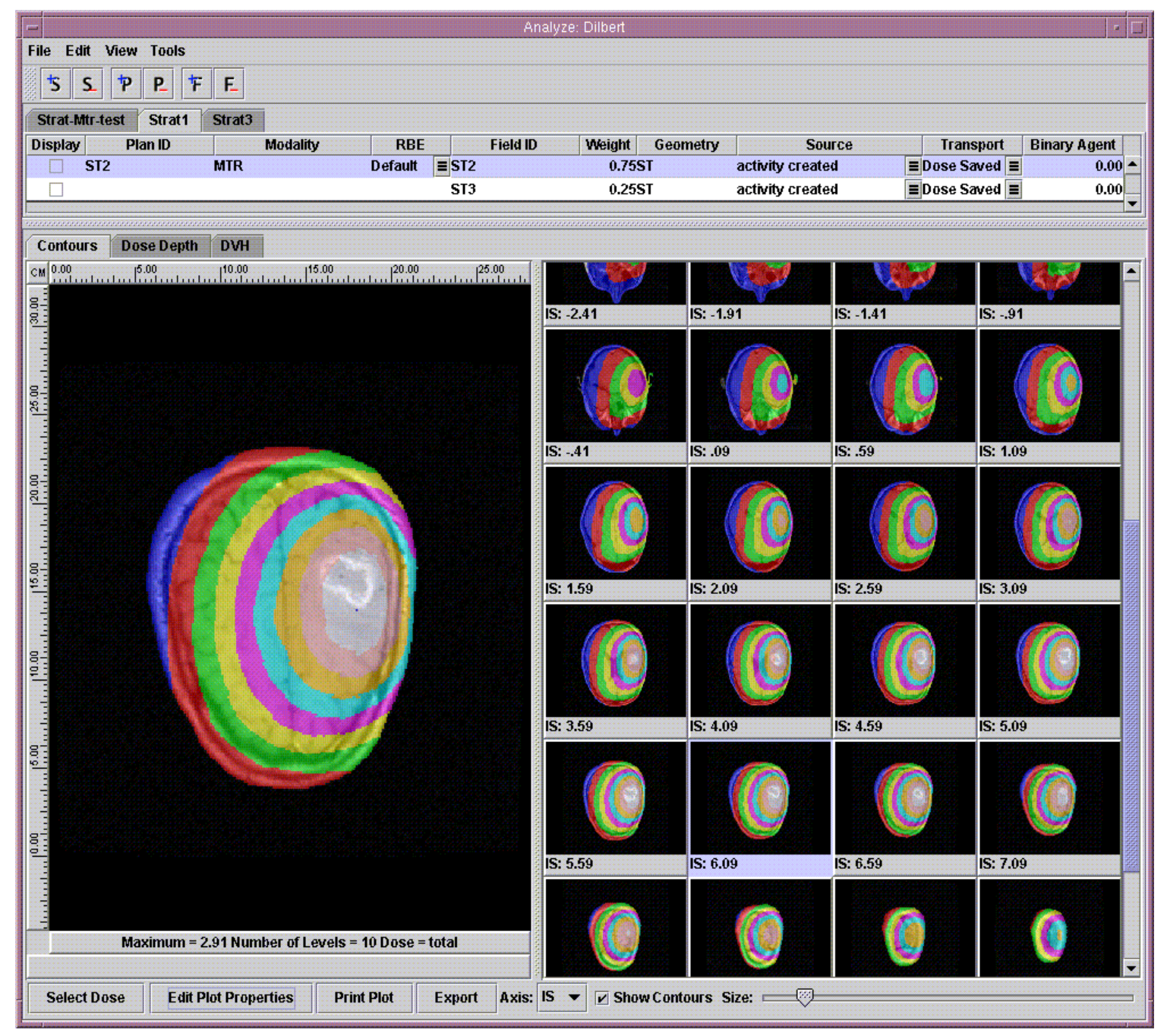

Figure 10. Analyze module user interface.

The Analyze module merges the dose data from multiple fields into one dose data object for a plan, and multiple plans are merged into a strategy. This merger can be customized by allowing the user to set modality-dependent biological weighting factors by field, region, and dose component. Binary agents, radioprotectants, and radiosensitizers may be included for each field, with the effects included in the merging process.

Analyze uses a plugin system to display the dose data to the user. This allows the user to customize the program by adding additional display plugins. The standard plugins calculate and display dose contours, dose volume histograms, and dose-depth charts. The dose volume histogram plugin itself uses plugins to generate the data and users will be able to implement their own algorithms for these functions.

The contour plugin will display the dose data as contours drawn on top of an image set, as shown in Figure 11, on all three principal axes. These contours will automatically update in the background as the user changes the weighting values (biological weight factors, binary agent concentrations, etc.) for interactive feedback. The user also has the ability to print and export the contours as an image file. 


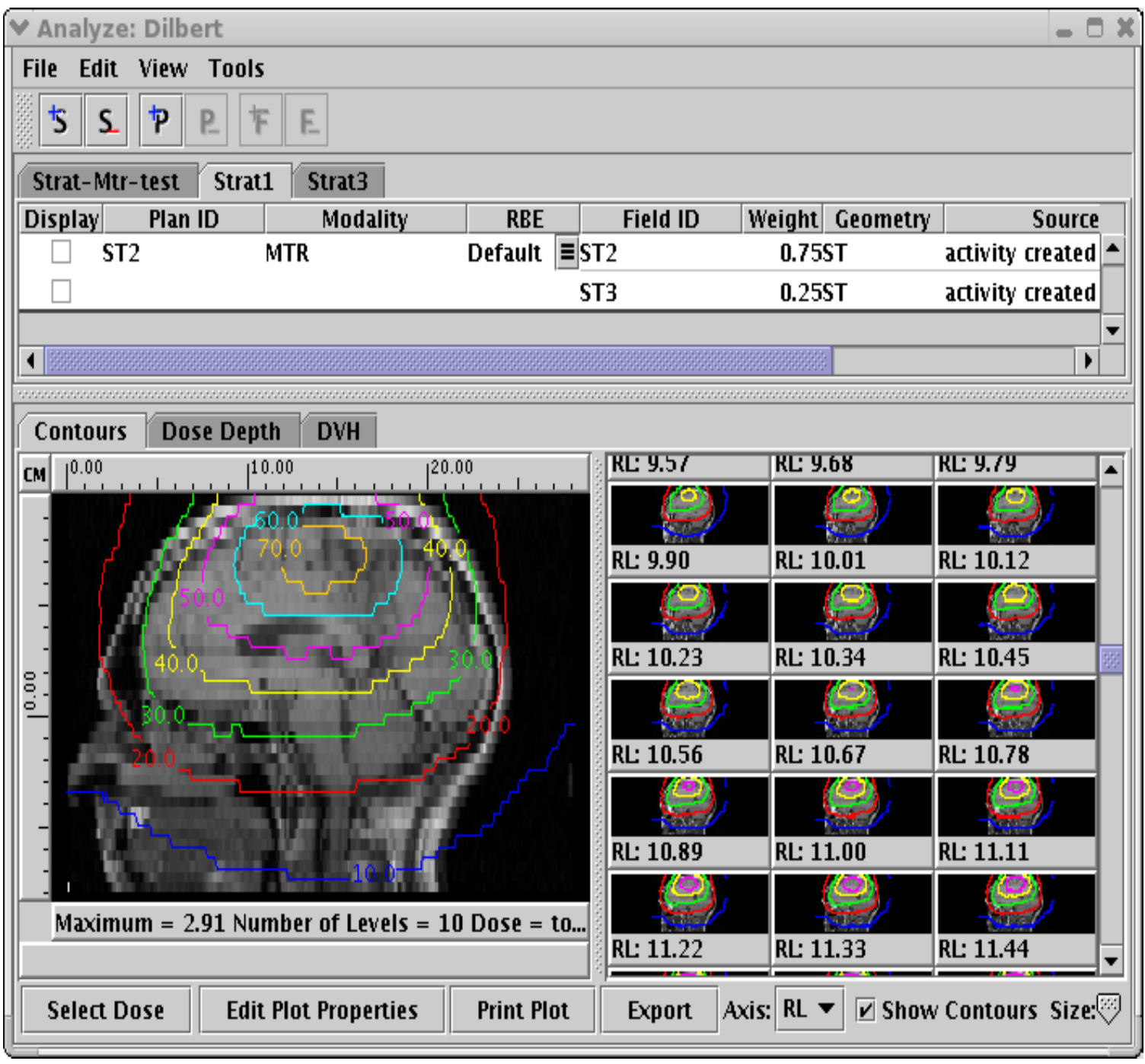

Figure 11. Analyze module dose contour display.

The dose volume histogram (DVH) plugin displays the dose information based on the chosen graph type, as shown in Figure 12. It currently supports several different plot types, including plugins for cumulative, frequency, and volume graphs. The dose-depth plugin will display dose information on a chosen line segment through a 3D region. Both plugins have automatic updates as the user changes weighting values. The user is able to print, export, or save plots to the database for future use. 


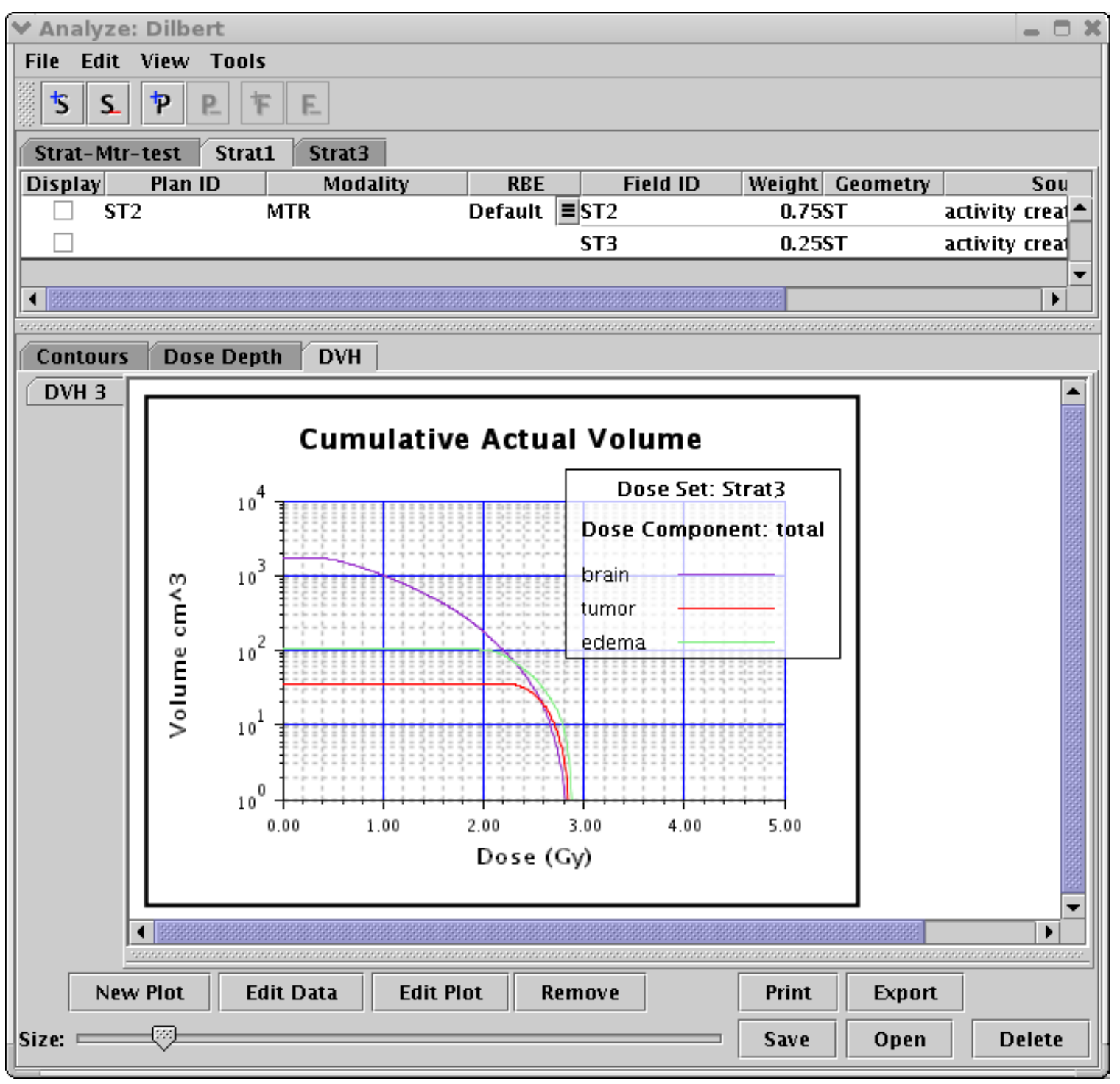

Figure 12. Analyze module DVH display. 


\section{INSTALLATION INSTRUCTIONS}

Before installing MINERVA, an ODBC/JDBC compliant database system must be installed. Instructions for setting up MySQL are given in Appendix A and instructions for PostgreSQL are given in Appendix B; to install other database systems, follow the instructions provided with the database software. MINERVA is distributed to the typical user as either an exe file for Windows users or a bin file for Linux, Solaris, or MacOS X users. After running or executing this file as is normal for your operating system, the wizard window shown in Figure 13 should appear. Simply follow the instructions and the run time version of MINERVA will be installed on your system. Appendix $C$ provides a list of additional software that must be installed for proper MINERVA operation; these packages should be installed before attempting to install MINERVA.

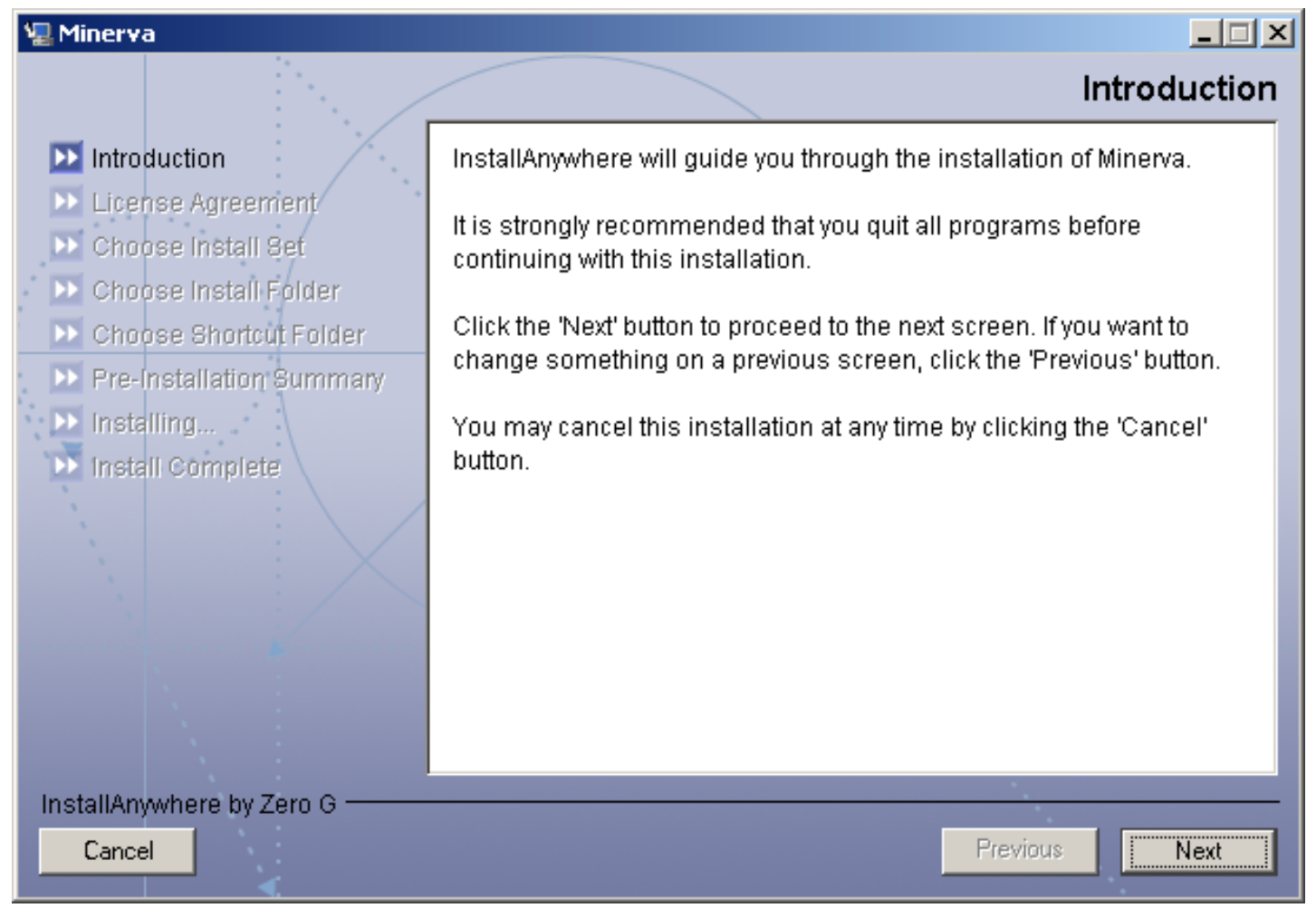

Figure 13. MINERVA installation wizard window. 


\section{MINERVA OPERATIONAL GUIDE}

It is not the intent of this section to exhaustively cover every possible selection in the various modules; that would be overly detailed and burdensome, and that function has been delegated to the MINERVA help system. Rather, the aim of this section is to provide an overview of the basic functionality needed by the user to start the various modules and access the help menu for further operational detail.

\subsection{MINERVA Help System}

As a supplement to the information provided in this manual, a help system has been developed for MINERVA. This system provides a help information facility that allows users to navigate, search, and display help information, as shown in Figure 14. It can also help new users learn how to use the MINERVA system, reducing the initial training time.

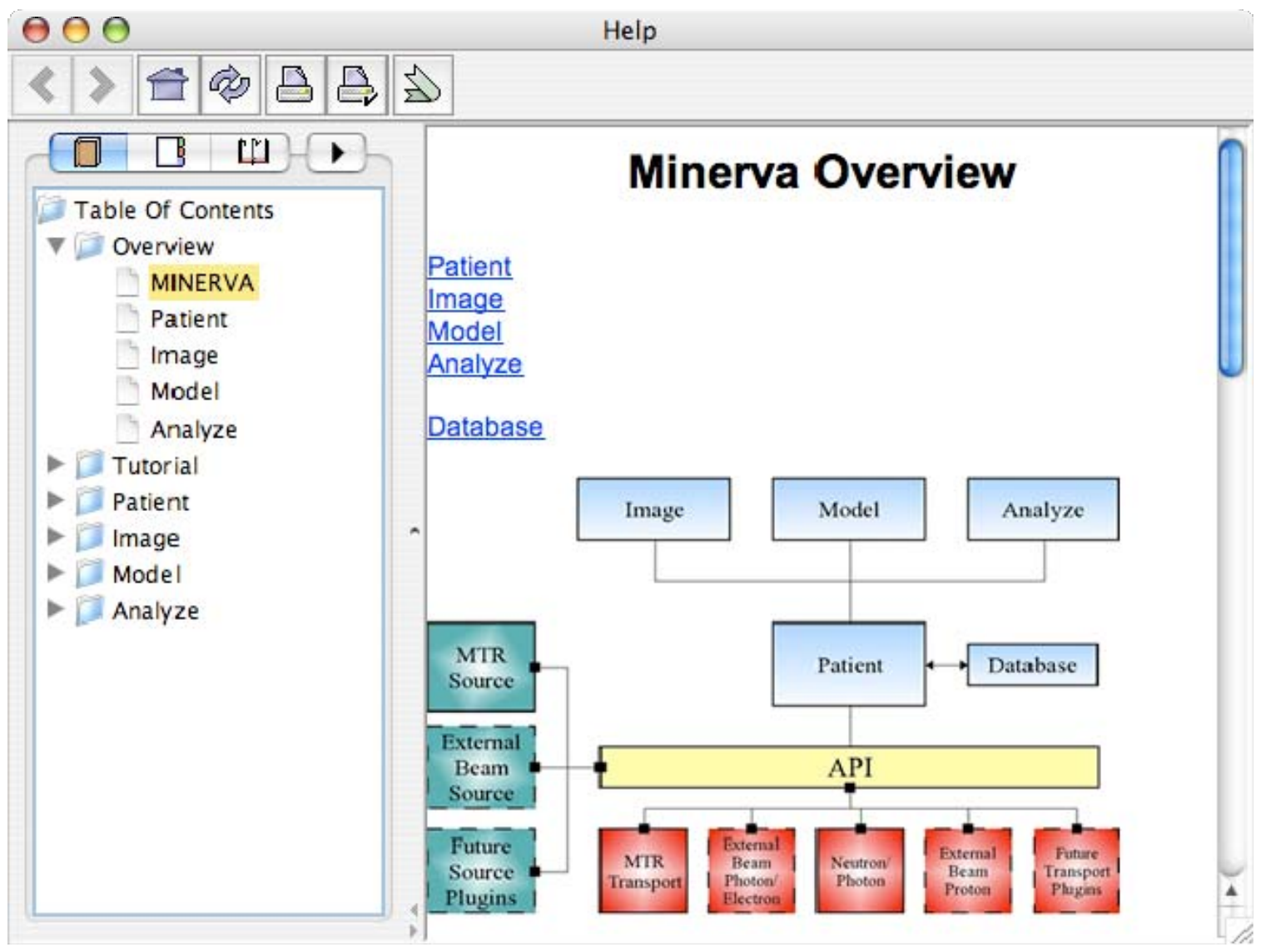

Figure 14. The MINERVA help system.

The help system has a table of contents displaying all the pages in a tree structure, an index, a glossary of terms, and a search function. Information for additional plugins can be added as needed, as can tutorials. The help system may be launched from any of the MINERVA modules or plugins. 


\subsection{Patient Module}

The Patient module provides access to the Image, Model, and Analyze modules and provides the interface layer to the patient database. This module can be launched from the command line or a desktop icon. Launching the Patient module should produce the display shown in Figure 15.

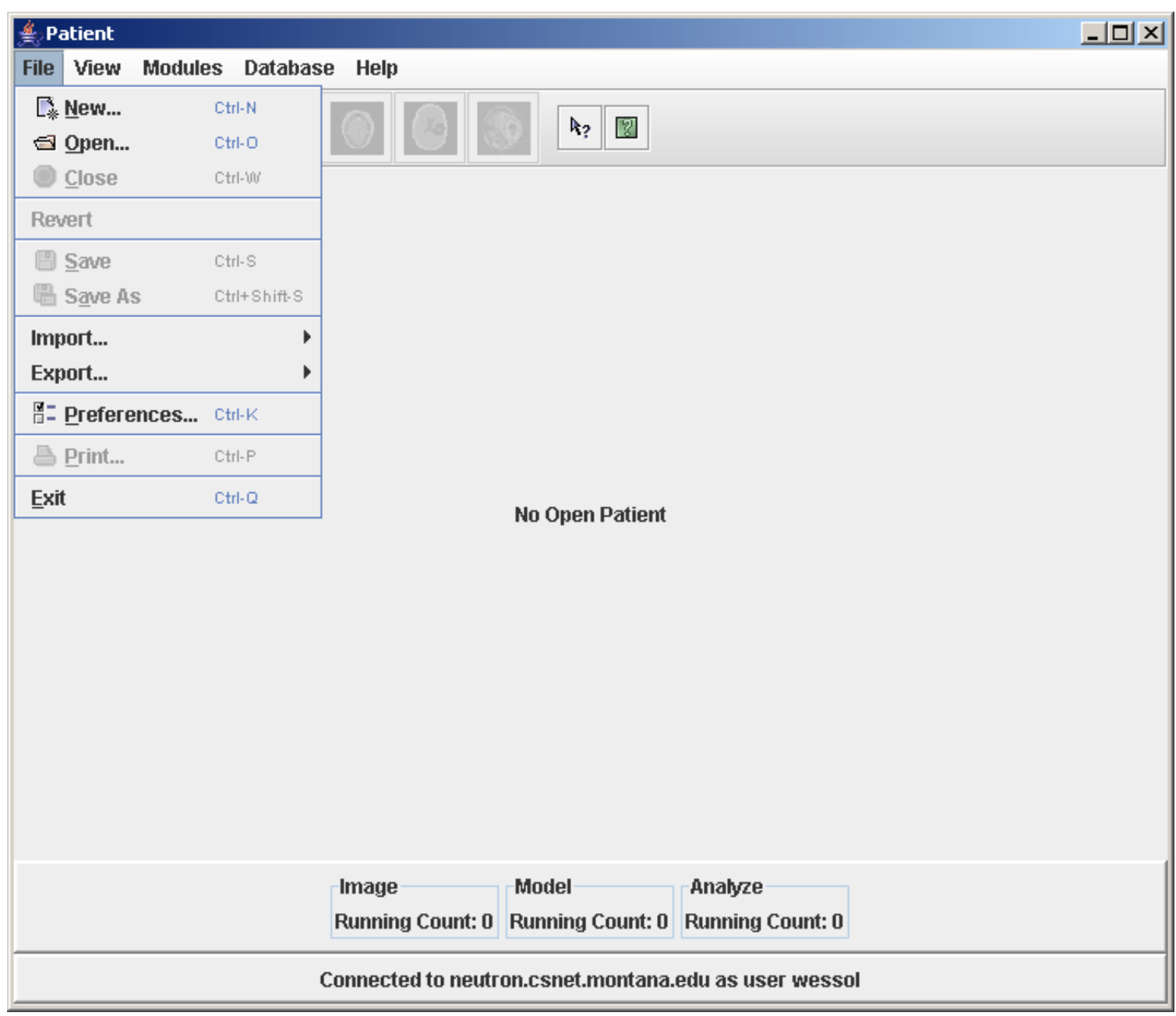

Figure 15. Patient module main interface.

To open a patient in the Patient module, select Open from the File pull-down menu. If connection to a database has not been made, you will be prompted to do so, as shown in Figure 16. 


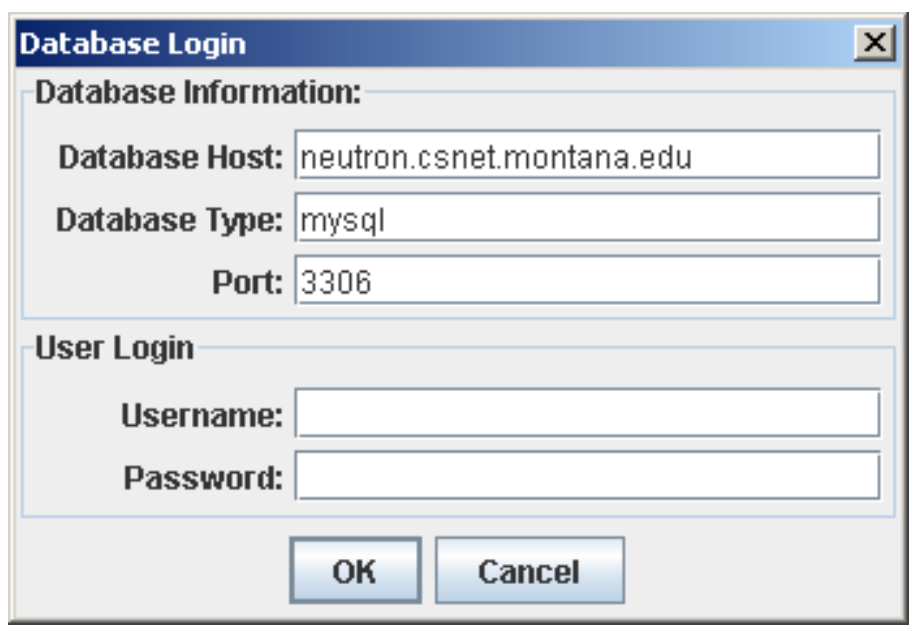

Figure 16. Interface prompting for database connection information.

Once connected to a patient database, choose the patient from the list and click OK, as shown in Figure 17.

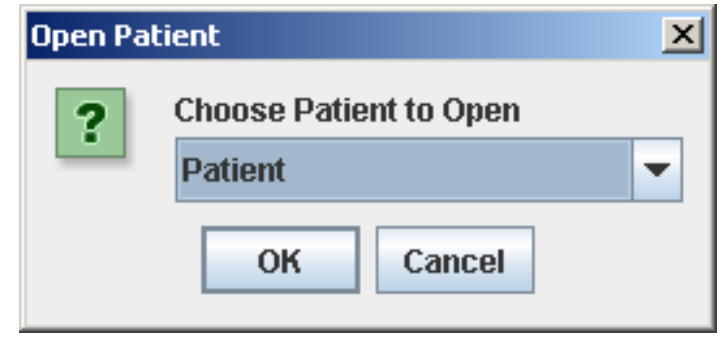

Figure 17. Selection of a patient from the database.

If opening a new patient choose New from the File menu and enter the new patient ID, as shown in Figure 18.

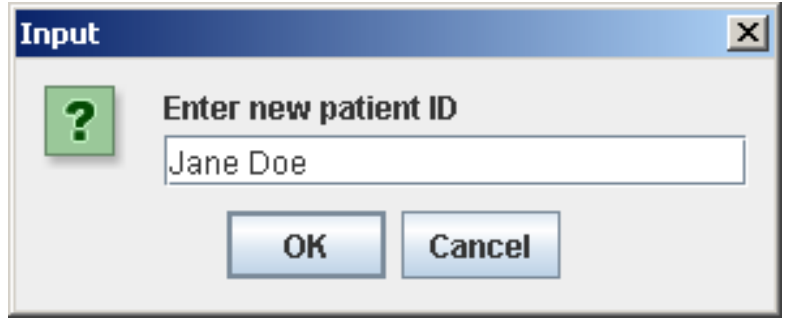

Figure 18. Entering a new patient ID.

If an existing patient ID is entered for a new patient, the Patient module will produce an error message. A new and unique patient ID must be provided for each patient. 
The patient information may now be entered, via the panel shown in Figure 19. This includes normal and emergency contact information, physician information, and patient medical history. A photograph of the patient may also be provided, if desired. Navigation between data fields may use either the TAB key or the mouse.

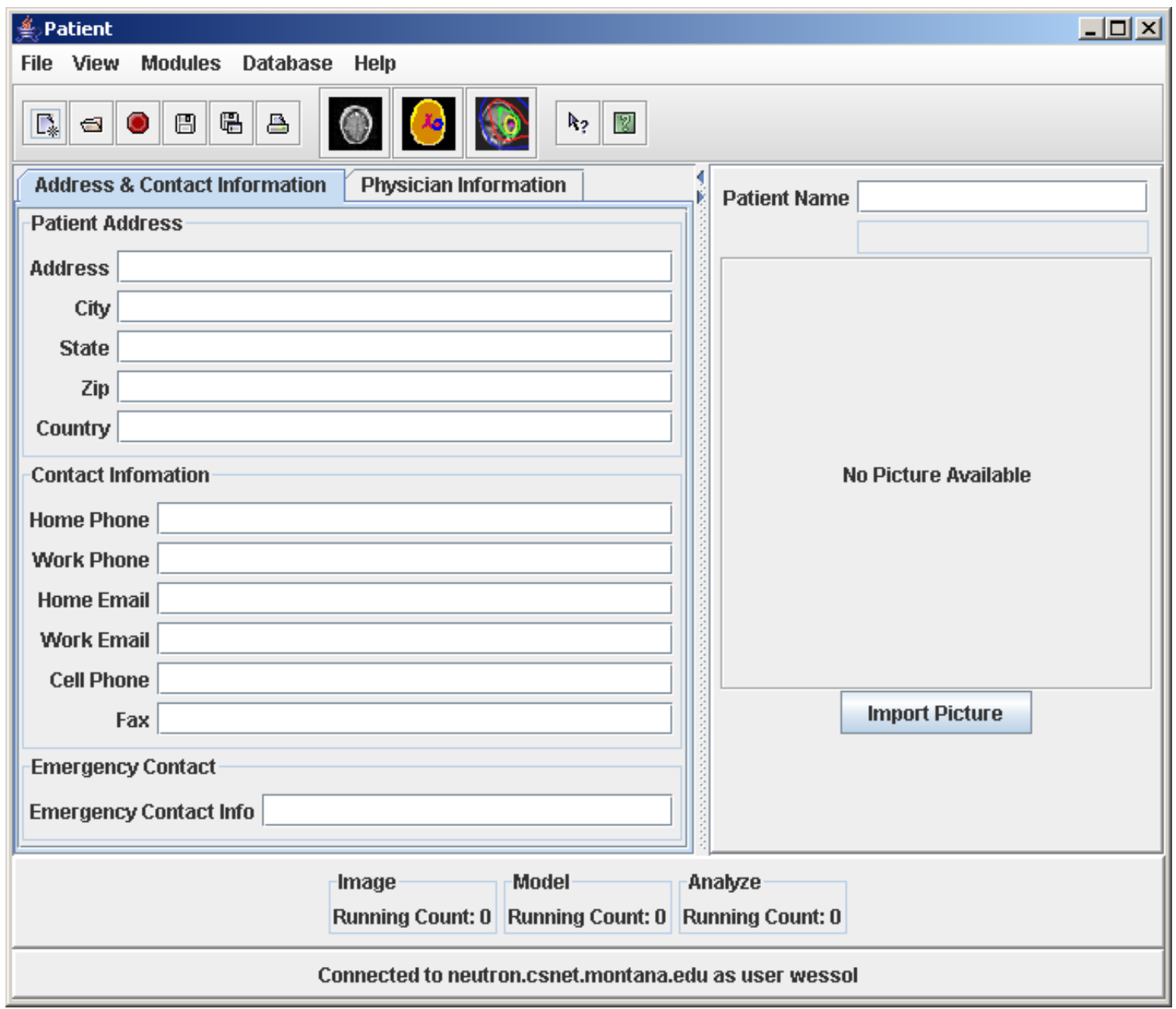

Figure 19. Patient information panel.

Importing and exporting data for a particular patient can be done from the Patient module. Data can be imported by using the option "Import New Patient" under the File $\rightarrow$ Import menu. This imports data for a new patient not currently in the selected database. Data may be added to an existing patient with the "Importing Into Current Patient" option, which can also be found under the File $\rightarrow$ Import menu. This operation imports particular data objects without having to import the entire patient. Exporting patient data can be done using the "Export Data" option located under the File $\rightarrow$ Export menu. This allows any particular subset of the patient's data to be exported.

All XML import and export operations use a dependency checking system to ensure data integrity. For example, if the user were to try to import or export a 3D model, then the Patient module would also require importation of the images that were used to construct that model. In order to allow this in an intuitive manner, MINERVA supplies a visual tool that allows the user to see the dependencies in real time. If the user selects an object that has dependents, then the dependents will also be selected. There is 
protection in place to prevent the user from unselecting any data object that is a dependent of another data object. If the user tries to unselect a dependent object, an error popup will be displayed (see Figure 20).

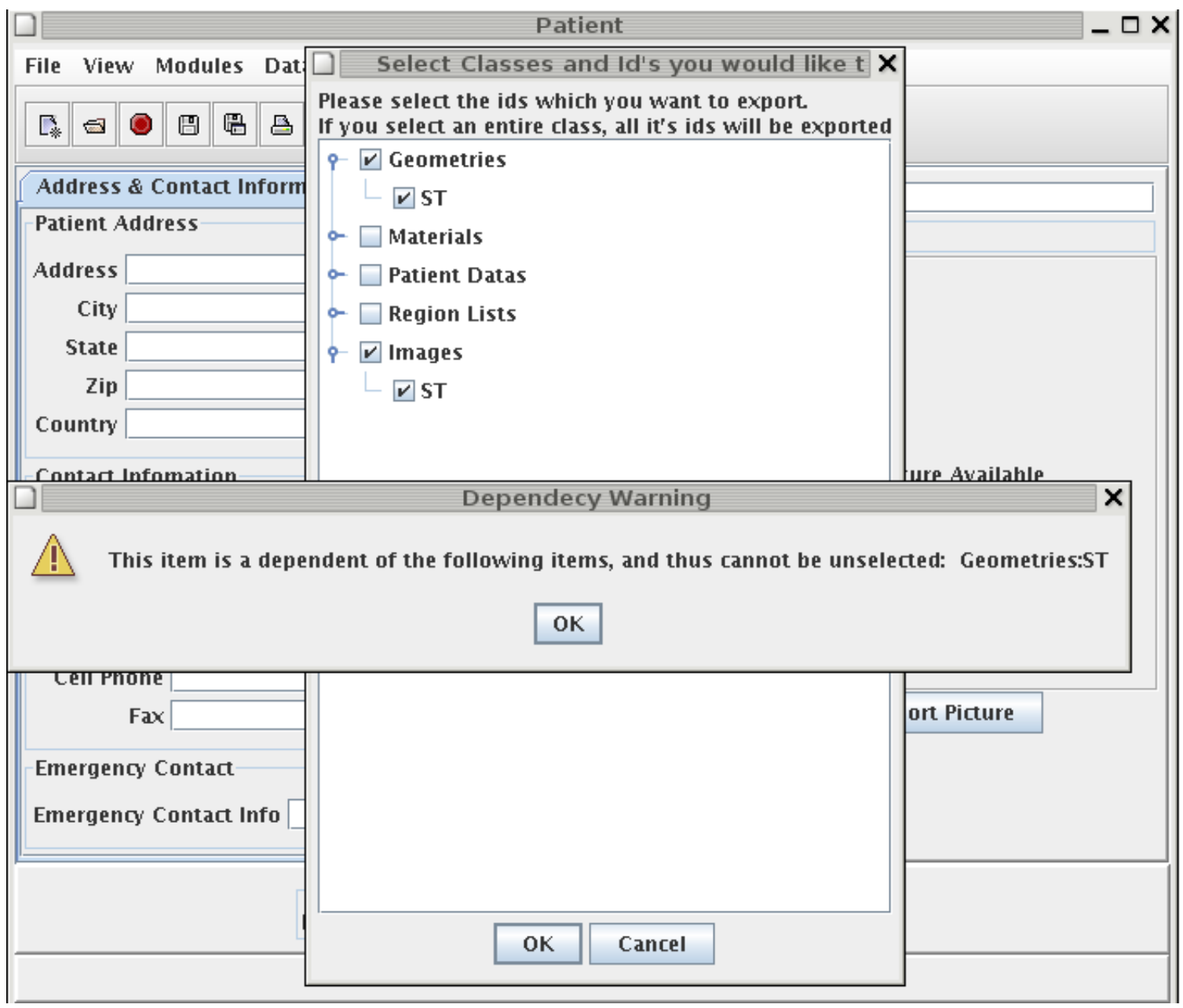

Figure 20. Export dialog and dependency error message.

The Import dialog looks virtually the same as the Export dialog, except the Import dialog has a different header. This helps the user identify which window is open.

\subsection{Image Module}

The Image module is launched from the Patient module through either the Image Module icon on the toolbar or the Modules menu. As stated in section 3, the Image module can currently Import DICOMRT, QSH, JPEG, and raw image. Standard image processing features, including noise filtering, gamma correction, and contrast enhancement, are also provided.

With the exception of the Register selection of the Tools menu, the help system should provide sufficient operational detail for the other Image module menu features.

A rigid body registration system based on maximization of mutual information (Viola and Wells 1997) has been included, with a three-dimensional display of the image registration process (Figure 21). To facilitate the registration, and to define the univel geometry, a reslicing tool has been developed. This tool 
performs a pixel-by-pixel interpolation between existing image slices to define new images and create a uniform spacing between image slices. This univel representation is the basis for the segmentation process in the Model module.

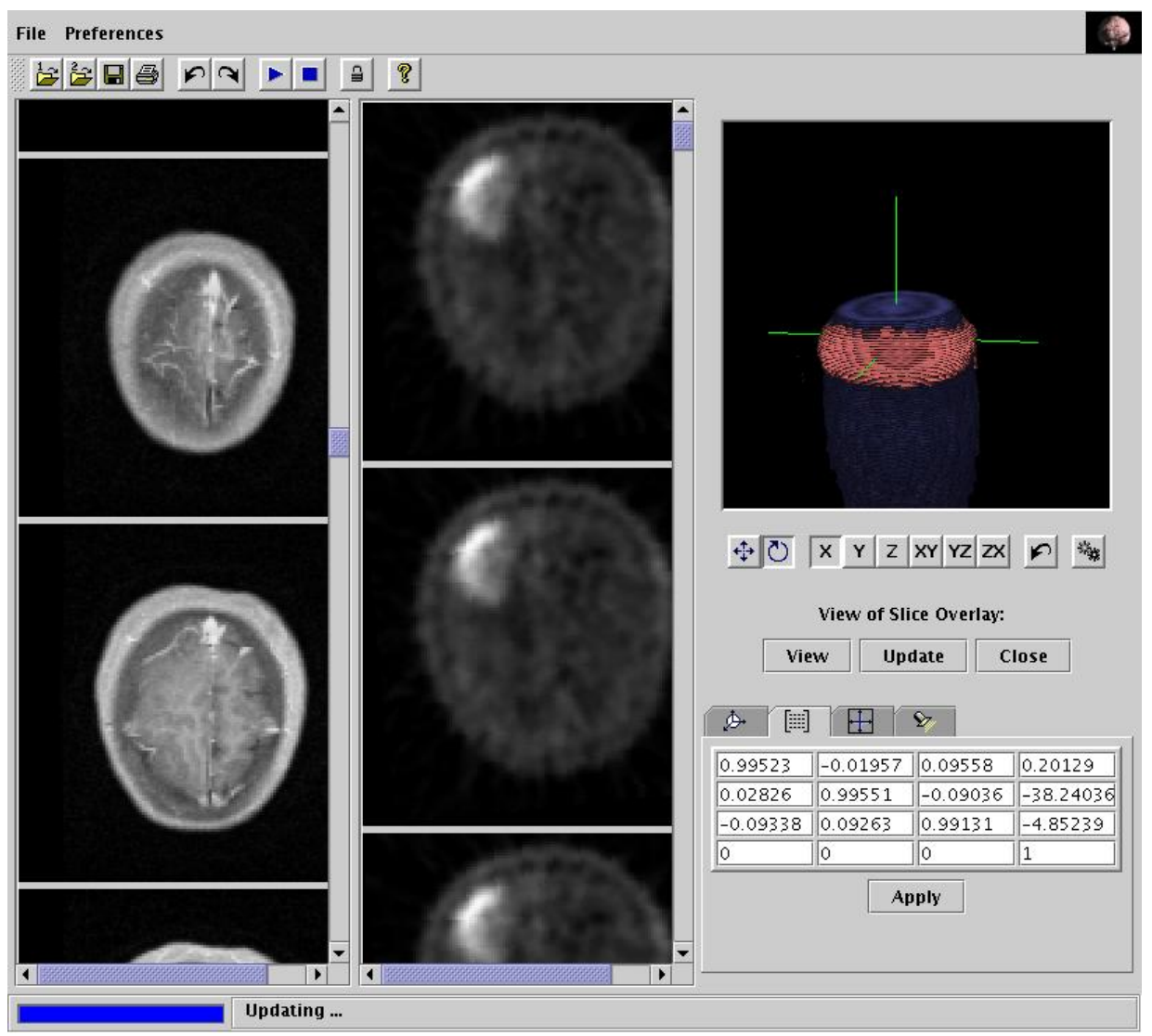

Figure 21. Display from the MINERVA Image module, showing co-registration of MR and PET images.

\subsection{Model Module}

The Model module is used to segment an image set by defining organs and other structures using a variety of segmentation tools. This defines the patient geometry model used to perform dose calculations.

The Model user interface is split into six main sections. The segmentation tools are displayed on the far left, with the currently active tool highlighted. These tools are provided by plugins, so this section of the display may look different depending on the plugins installed. To the right of the tools display is the main editing area. The currently selected tool is operable inside this area and any actions taken (mouse clicks, drags, etc.) are forwarded to the current tool. Farther to the right is a thumbnail display of the image slices. Clicking on a slice will place that slice in the editing area. The list of currently defined regions within the geometry is at the far right. (In MINERVA, a region denotes an organ or structure and the properties - composition, density, etc. - associated with it.) The view controls, including zooming, axis orientation, and opacity, are at the bottom center. The options available for the currently selected tool are displayed at the bottom right. These options will change depending on the selected tool. 


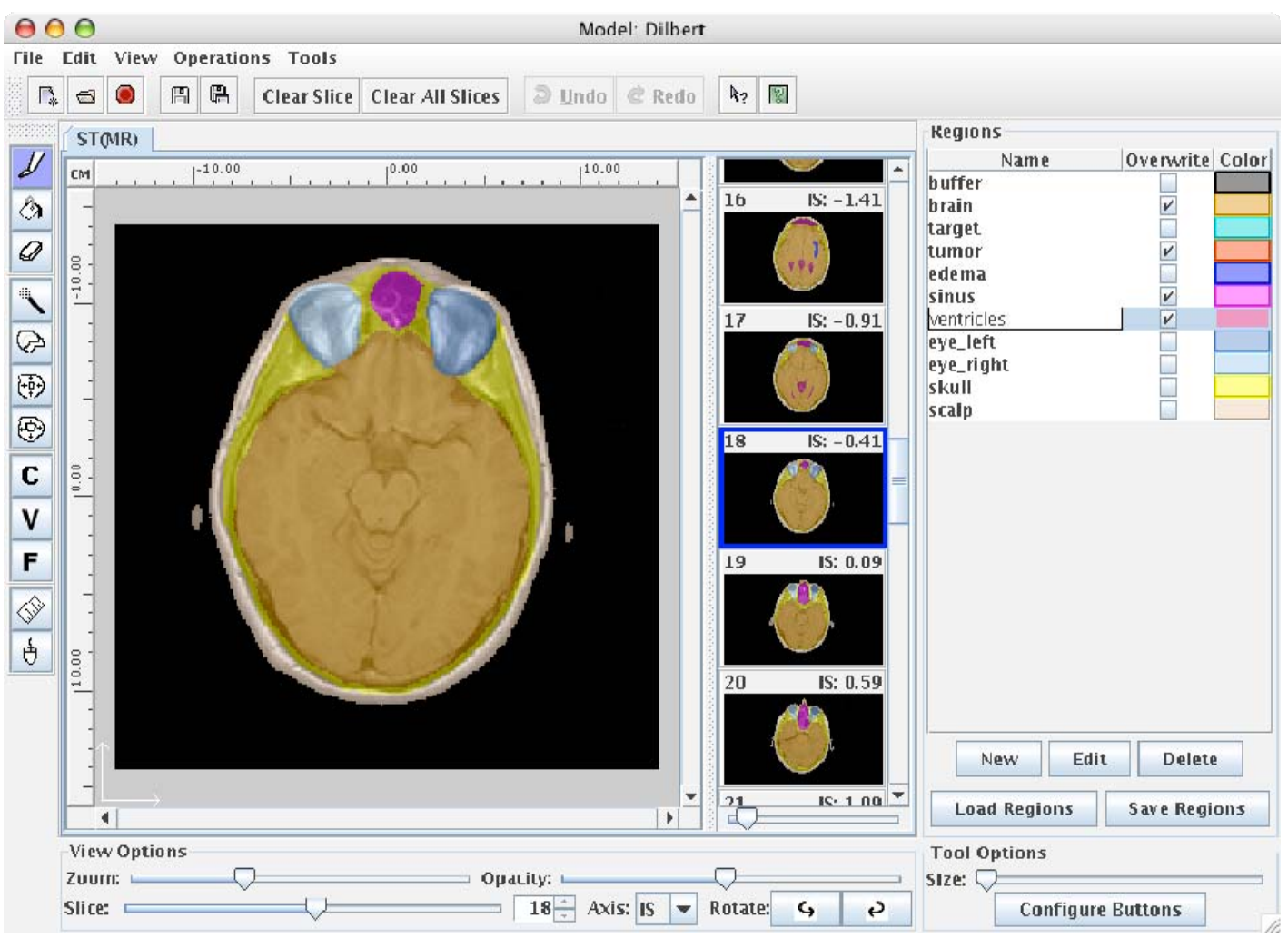

Figure 22. Model module user interface display.

\subsubsection{Editing}

While segmenting, it is important to remain aware of the current region and the status of the overwrite option for that region. The current region is the currently selected region in the regions list. The tools use the current region when performing their operations. The overwrite flag allows the user to protect some or all regions from being accidentally erased or overwritten. If the overwrite flag is turned off, then the univels with that region value cannot be modified. In order to erase or alter part of a region, that region's overwrite flag must be set.

\subsubsection{Tools}

The segmentation tools along the left hand side of the window are used to define structures within the geometry. If the selected tool has any configuration options to control its behavior, then the controls are placed in the lower right of the Model display.

Paintbrush: Paints the area under the mouse (drag) with the currently selected region. The brush size can be controlled and the middle and right mouse buttons can be defined to operate other tools. It is useful to define the middle button to use the Fill tool (Paint Bucket) and the right button the Eraser tool. In this way you could quickly outline an object, fill it, and make corrections by erasing.

Paint Bucket: Fills an enclosed area with the current region. Usually the paintbrush is used to outline a region and the paint bucket is used to fill the interior of the outline. The paint bucket can 
also be used to change the region assigned to a segmented area. If the user clicks on a segmented area with a different region selected, then the area they clicked on will be replaced with the currently selected region. The paint bucket can work in 2D or 3D mode. In 2D mode the fill is constrained to the current slice. In 3D mode the tool will fill connected areas above and below the active slice.

Eraser: The eraser is the inverse of the paintbrush - it removes the region definition from the selected area under the mouse. This tool uses the same brush size as the paintbrush.

Wand: The wand tool fills based on the image pixel intensity. The user selects a "seed pixel" that serves as the starting point for the wand function and defines a threshold for the pixel intensity search. The wand tool will expand the area around the seed pixel, in either $2 \mathrm{D}$ or $3 \mathrm{D}$, to include pixels directly connected to the seed pixel that have intensity within half the threshold (plus or minus) of the seed pixel intensity. This expansion is continued recursively until either no additional pixels within the threshold are found or until a boundary, either a defined area with overwrite protection or the image boundary, is encountered.

Copy: Allows the user to copy selected areas to a different slice. When the user clicks on a region in the image, the copy tool finds the boundary of that region and copies it to the clipboard. To select multiple areas hold down the shift key while selecting regions. A preview is displayed in the tool options area to show the clipboard contents.

Erode: The erode tool will find the edges of the region the user clicked on and remove a specified amount from the edge of the region. The amount removed can be specified in pixels, centimeters, or millimeters. The erode tool can be constrained to work only in the current slice or to work in $3 \mathrm{D}$.

Dilate: The inverse of the Erode operation. Instead of deleting pixels at the edge of the region, the dilate tool adds pixels. Like the Erode tool, the amount added is user-defined and the tool can be configured to work in $3 \mathrm{D}$.

c Constraint Marker: This tool allows the user to specify constraints on any point in the geometry or for any region. The constraints allow the user to specify limiting doses for that location or region.

V Convergence Marker: The convergence marker allows the user to specify locations in the geometry to be used to determine dose calculation convergence in the transport plugins.

F Fiducial Marker: The fiducial marker allows the user to mark points in the geometry for use in registration, alignment, or positioning.

\subsubsection{Operations}

Operations are commands that affect the entire geometry rather than just a single slice.

Mode Fill: This operation will search the geometry for unassigned pixels. For each unassigned pixel, it will find the region that has the most pixels surrounding it and assign that region value to the unassigned pixel. This is useful for catching the occasional stray pixel that was missed by a tool operation. 
Thresholding: The thresholding operation allows the user to isolate regions using pixel intensity thresholds. The threshold can apply to a slice or to the entire image set. It may also be applied to a subsection of a slice by clicking on the preview (see Figure 23) and the threshold operation will flood fill from that point, constrained by the threshold. To specify the threshold the user clicks and drags in the histogram area. The preview image will update to display the results of the thresholding operation. The user can modify the threshold definition by holding down a modifier key. A slider mode is also available to define the upper and lower threshold values.

\subsubsection{Loading Data From External Files}

The Model module provides two plugins which read geometry information from existing files. Under the File menu is a Import menu that lists all the current plugins for importing data. The first plugin, labeled UVH on the menu selction reads univel geometry files, and the other plugin, labeled Dicom Geometry, has limited support for DICOM RT structure sets that have closed planar contours as long as the image set and the structure set are within the same plane.

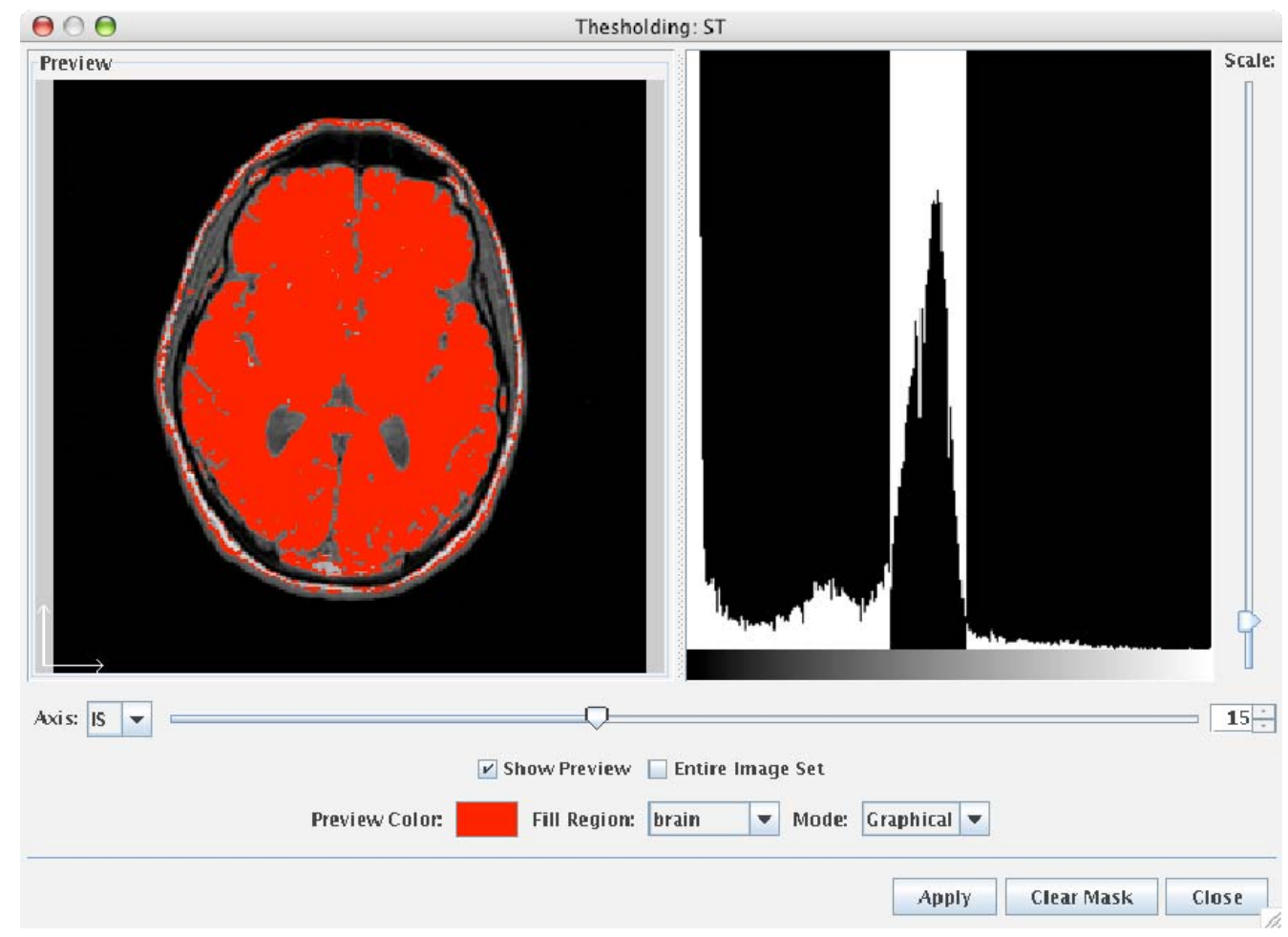

Figure 23. The threshold operation display.

\subsection{Analyze Module}

The Analyze module has two principal functions: create one or more treatment plans for a patient; and graphically display and compare the dose data from these various treatment plans. The Analyze module display window has a specific work area for each of these functions - the upper section is used to define treatment plans and the lower section displays the dose data. 


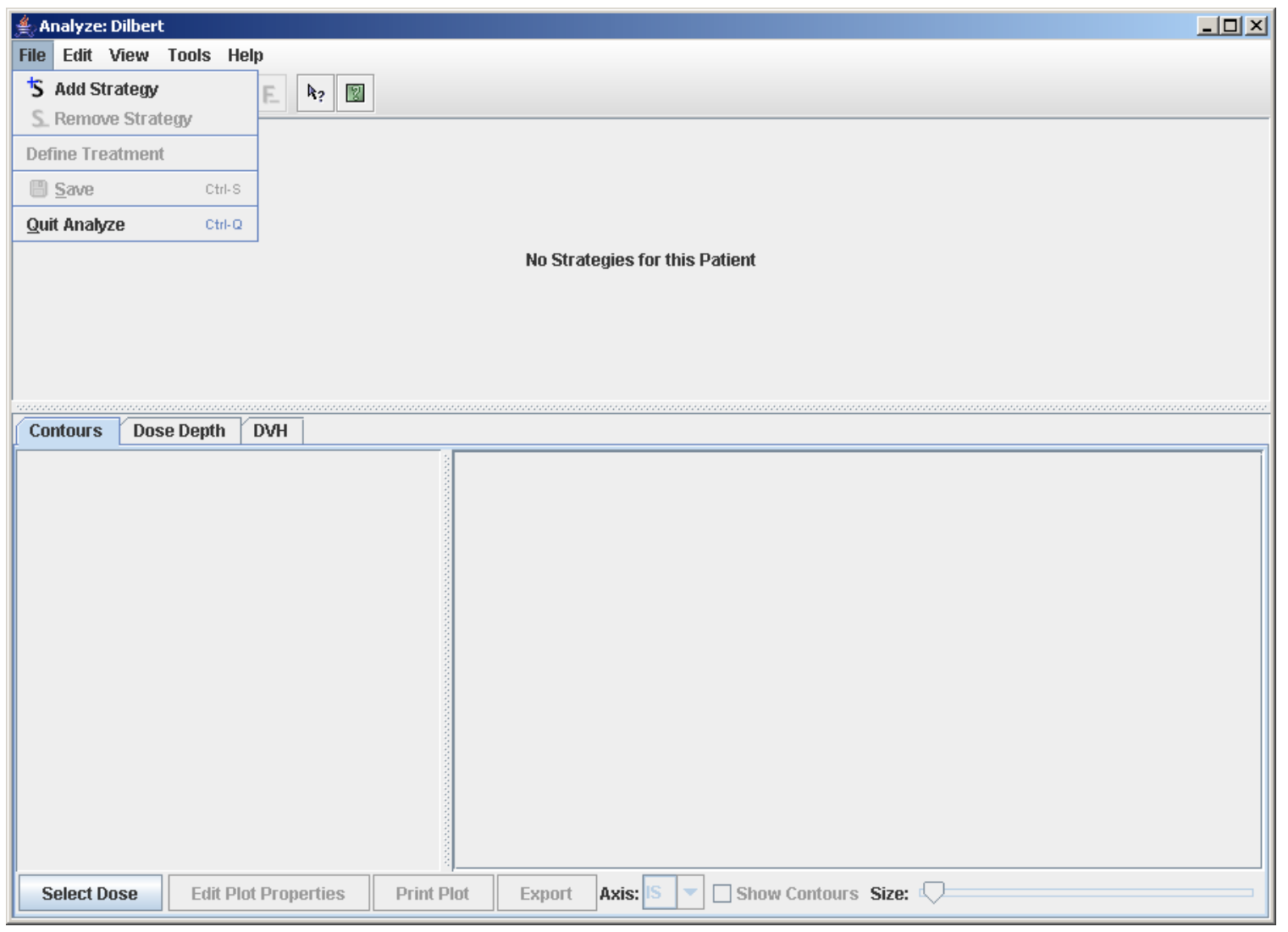

Figure 24. Analyze module interface display.

\subsubsection{Definitions}

The following terms need to be defined to allow a clear and consistent explanation of the Analyze module functions.

Field: A field is the base data object. It represents a single source of radiation, and the effects of that radiation dose. Fields are used to build plans, which are then used to build strategies. A field may exist in more then one plan, so any changes to one field may affect multiple plans and multiple strategies.

Plan: A plan corresponds to a full treatment course with a single irradiation modality. A plan may be constructed from multiple fields, with the constraint that all the fields have the same coordinates (image sets are co-registered) and the same modality as the plan. Fields within a plan can be weighted to change the effect of a single field. A single plan can be inserted into multiple strategies. If a plan exists in multiple strategies, then all associated strategies will be changed if the plan is changed.

Strategy: A strategy is a complete treatment plan for a patient. It consists of multiple plans that may contain multiple fields, with the constraint that all plans in the strategy must have the same coordinates. Plans of different modalities can be added to the same strategy. Multiple strategies can be made and the user can compare the effects of the different strategies by evaluating the 
resulting dose data. Once a course of action is determined, the strategy to be administered can be locked to prevent any changes. This locked strategy is defined as the treatment.

Source Editor: Each field is associated with a single radiation source. The source editor is used to define the radiation source for the field.

Transport Editor: Each field requires a dose calculation. The transport editor takes the field definition (geometry, source, etc.), simulates the radiation transport through the geometry, and returns a data object containing the results of the simulation, i.e., the dose.

\subsubsection{Analyze Workflow}

The Analyze module has a specific workflow. First a strategy is created, and then plans and fields are added to it. The various source and transport editors are used to run the radiation simulations. Then, the user evaluates the strategies, plans, and fields using the dose display features. If a strategy/plan/field is found to be lacking, the user can go back, make changes, rerun the simulations, and revaluate the plots. This is done repeatedly until the user is satisfied with the results and can mark one strategy as the strategy used to treat the patient. This strategy will then become locked and the user will be prevented from changing it.

\subsubsection{Analyze Walkthrough}

This section describes the process for creating and adding strategies, plans, and fields.

1) First, create a new strategy by clicking on the new strategy button in the toolbar, or by selecting "New Strategy" from the File menu (see Figure 25).

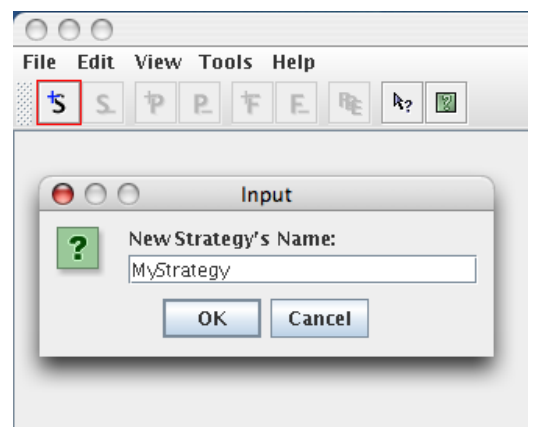

Figure 25. Display for creating a new strategy.

2) Next, create a plan by clicking the add plan button or selecting "Add Plan" from the Edit menu, and then clicking the "Create New Plan" button (see Figure 26). The plan treatment modality then must be selected. After creating the plan, click OK to add the new plan to the current strategy. To add the same plan to another strategy, click the add plan button again and, rather than creating a new plan, select one of the plans available in the plan popup menu. 


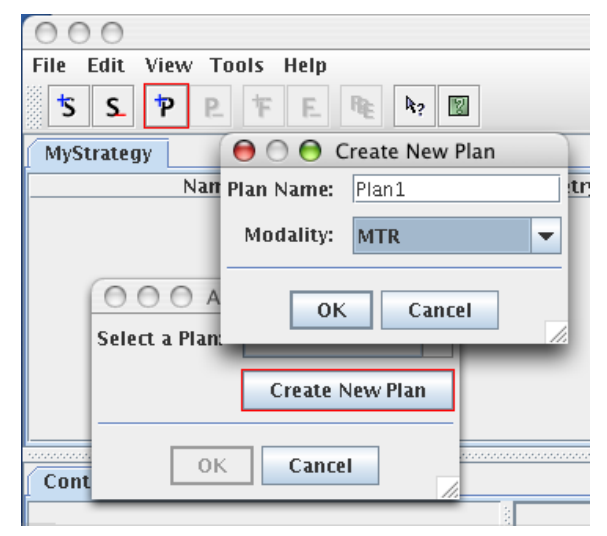

Figure 26. Display for creating a new plan.

3) Create a field by first making sure the plan created in the previous step is selected. Then click on the add field button or select "Add Field" from the Edit menu, and click the "Create New Field" button (see Figure 27). The add field button is context sensitive and will not be available unless a plan is selected. Create the field by giving it a name, the geometry to use, and specifying the modality, the source editor to use, and the transport editor. The geometry will define the coordinates for the field and Analyze will prevent addition of a field to a plan with different coordinates. Like the add plan command, the add field command will allow the same field to be used by multiple plans.

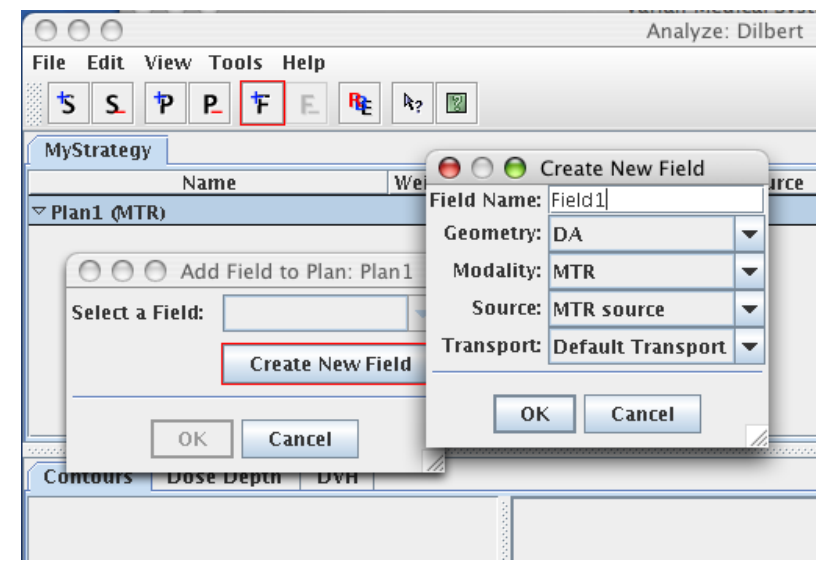

Figure 27. Display for creating a new field.

4) Once the field has been created the source and transport editors must be run. To run the editors, click the button next to the status message supplied by the editors. Each modality will have a different source and transport editor; these are defined locally.

5) As shown in Figure 28, the weight column for Field1 is highlighted in red. This is to alert you that the weights for that field do not sum to one. You will be prevented from plotting the results until the weights have been corrected. To edit the weight, double click on the desired weight value and enter the new value. 


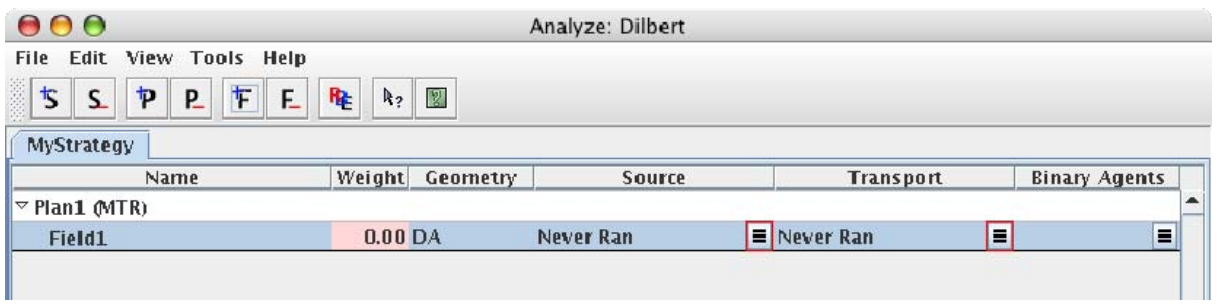

Figure 28. Analyze display showing a strategy with one plan and one field.

6) After the source and transport editors have run and the field weight values are set, the plotting features can be used to display and compare the dose data.

\subsubsection{Plotting}

In order to evaluate the effectiveness of a strategy/plan/field, the Analyze module includes three basic plotting engines. The Contours engine displays dose contours on top of an image set, Dose Depth traces a ray through the object's coordinate system and displays the dose levels along that ray, and the DVH, or Dose Volume Histogram, engine charts the volumetric distribution of dose. Each plotting engine is provided by a plugin, so new plotting plugins can be added easily.

The user can plot the results for an entire strategy, a plan, or just a field. There are some conditions that will prevent a plot from being generated. For example, if the transport editor has yet to be run for a field, then no plots for that field can be displayed. This cascades up the chain of objects, so a plan that contains an incomplete field cannot be displayed and the strategy containing that plan is also prevented from being displayed.

In order to display a plot, the user just has to click on a field, a plan, or a strategy. If the user's selection contains a valid dose, as described above, then it will be displayed in the current plotting mode. To switch between the different plotting modes, the user needs to select a different plot engine tab in the plotting area. As the user selects different objects the plots will update to reflect the user's current selection. Sometimes there may be a delay while the dose information is being calculated. During these calculations a short message will be displayed in the upper left of the plots informing the user that MINERVA is busy calculating the dose information.

\subsubsection{Contours}

The Contours plotting engine displays dose contours on top of an image set. To display the contours, simply select the "Contours" tab in the plotting area, and click on a field, plan, or strategy containing valid dose information. The resulting display will resemble that shown in Figure 29. 


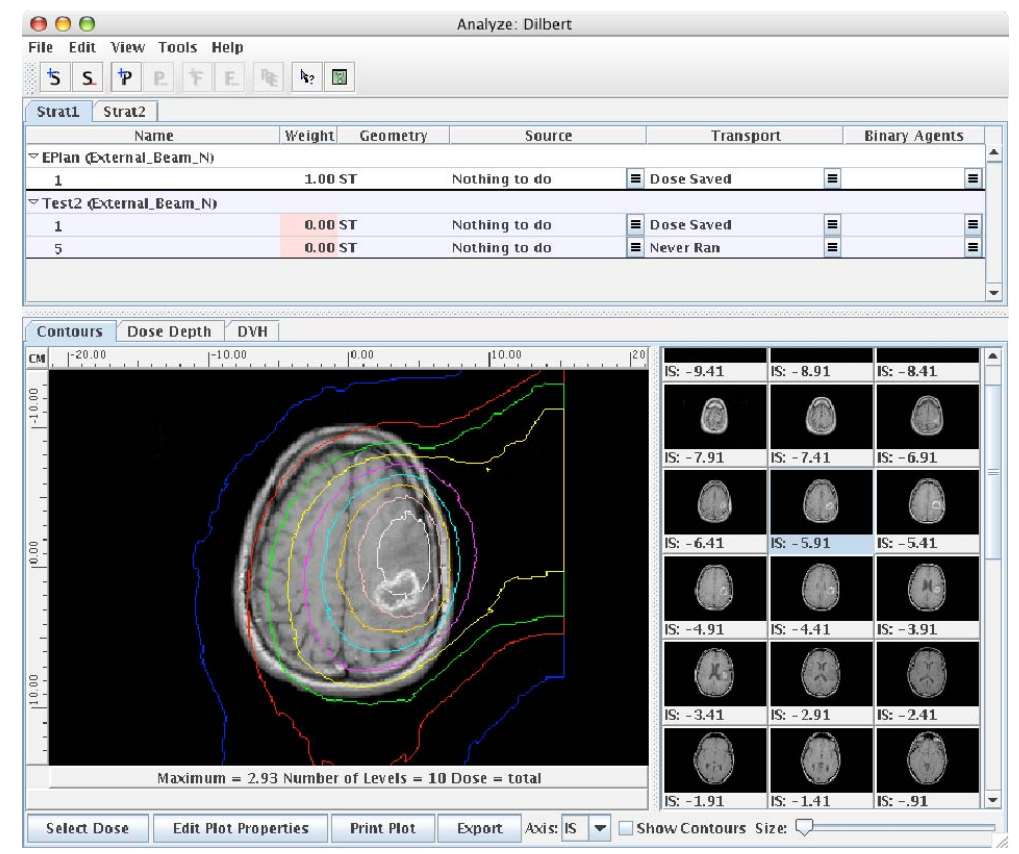

Figure 29. Dose contour display.

Thumbnails of available slices are displayed on the right hand side, and the large image is displayed on the left with the contours drawn on it. The contours can be displayed on the thumbnail images by clicking the "Show Contours" button on the bottom of the screen. The Contour data can be displayed along any of the three principal coordinate axes by choosing the axis from the Axis popup menu at the bottom of the screen (see Figure 30).

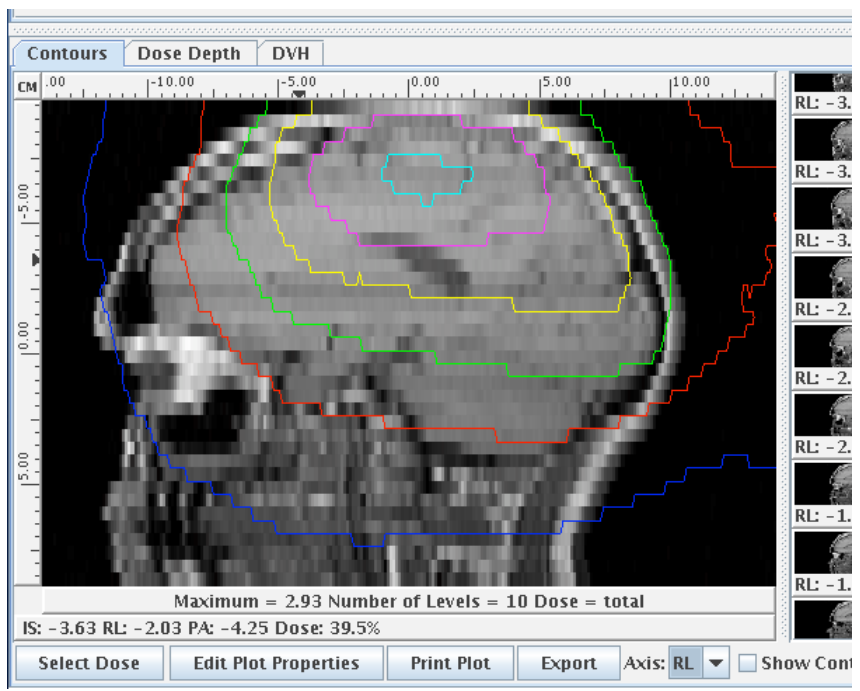

Figure 30. Dose contour display along an alternate coordinate axis. 
Various plot settings are available by clicking the "Edit Plot Properties" button (see Figure 31). These include configuring the ruler, displaying a grid, legend, adding labels, defining line styles, and specifying contour levels for display.

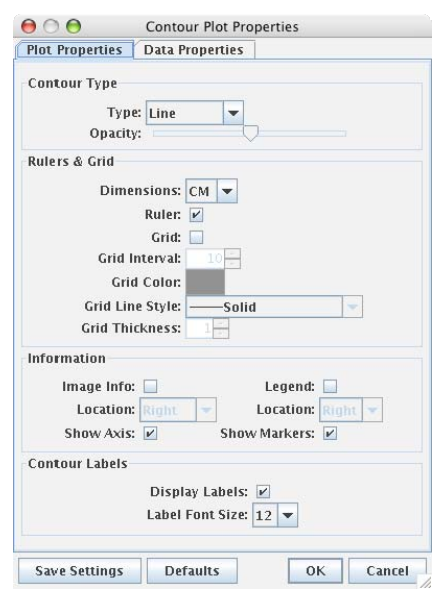

Figure 31. The Edit Plot Properties display for the contour plots.

In addition to the standard line contours, the Contours plot can be configured to display the doses as a color wash instead of lines, as shown in Figure 32. Finally, the plots can be printed or exported to a graphic file.

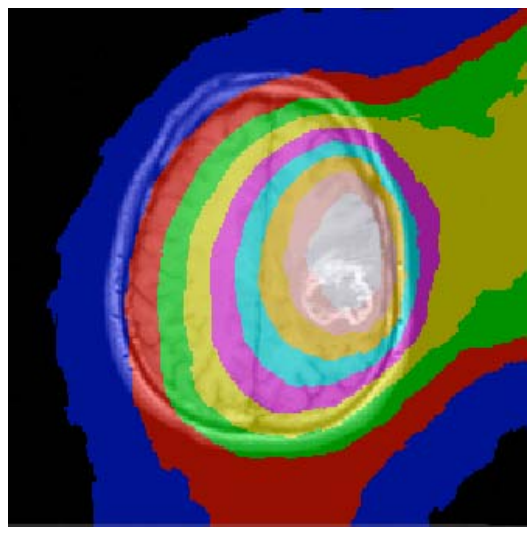

Figure 32. Colorwash contour display.

\subsubsection{DVH}

The DVH plot displays information about how much dose a certain region received. To create a DVH plot, click on the "New Plot" button of the DVH panel. This brings up a panel to select which dose components and which regions to graph. Multiple dose components, or multiple regions can be selected, but there cannot be both multiple selected dose components and multiple selected regions. The user interface will prevent the user from selecting multiple elements from both types. Options are also available to specify the type of DVH plot (cumulative or differential). The different DVH calculation algorithms are implemented as plugins, so new algorithms can be added to the system easily. 


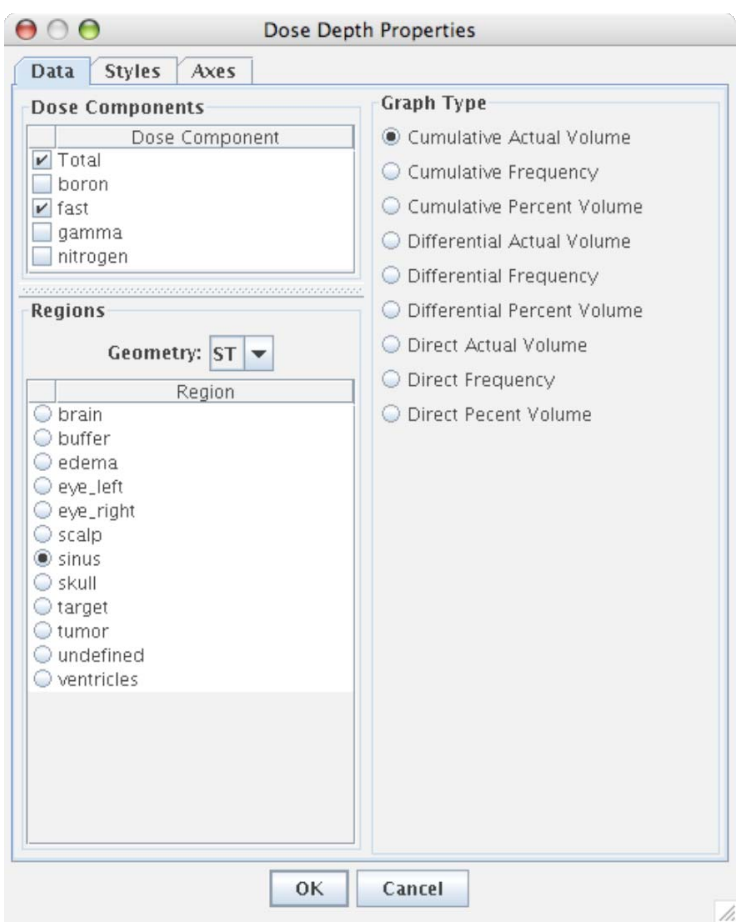

Figure 33. Colorwash contour display.Figure 33. Colorwash contour display.

After the wizard completes its operations, the plot will be displayed. In addition to the plot, a tabular summary of the DVH statistics is provided.
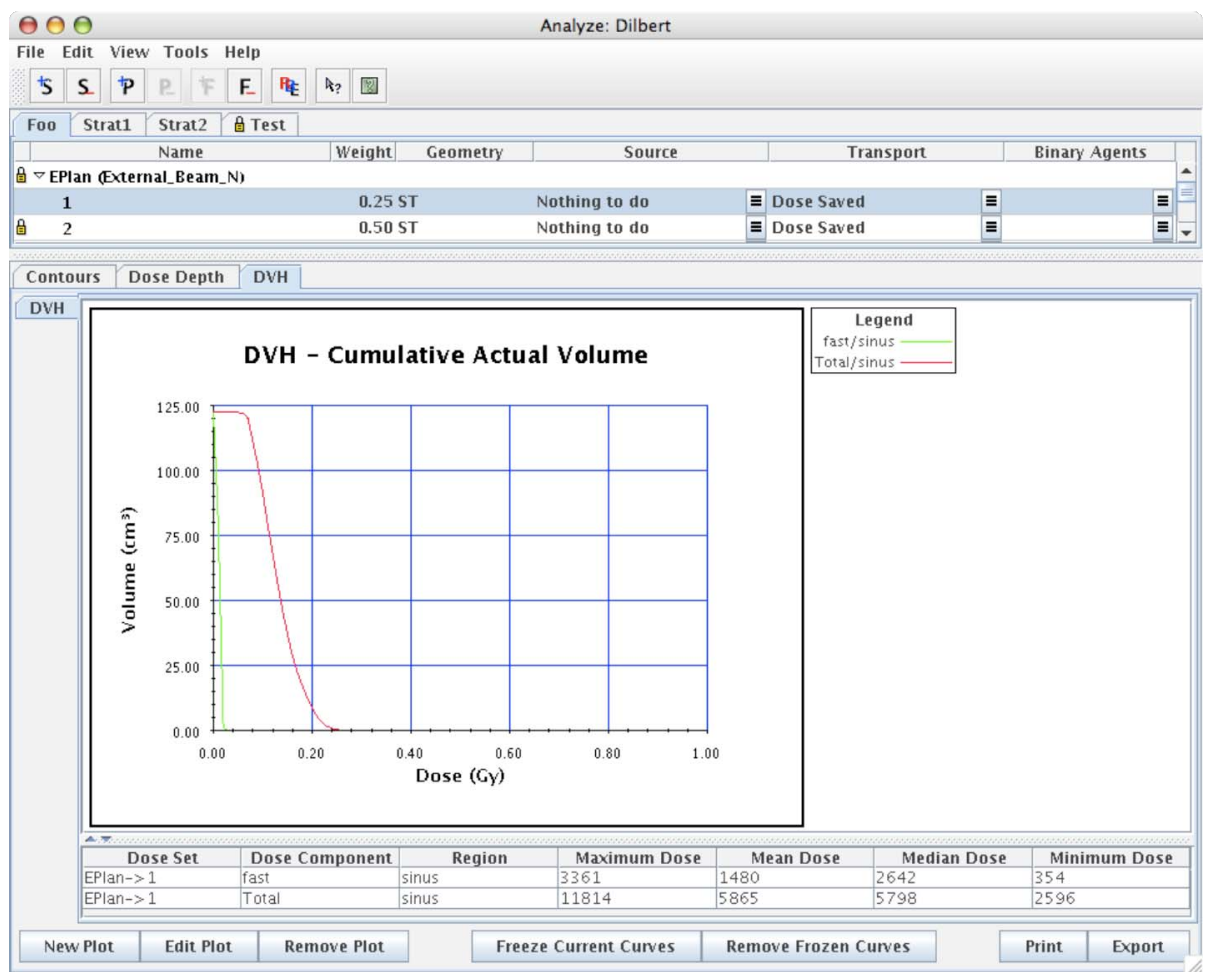

Figure 34 . The DVH plot display. 
Clicking on the "Edit Plot" button allows the user to re-define the data sources and modify the properties of the plot (line color, range, title, etc.). Like the Contours plot, the DVH plot allows you to print and export to a graphic file.

In order to compare two or more fields, plans, or strategies, the current curves can be frozen. This causes the current curves to stay on the plot as the user selects different fields, plans, or strategies. The curves for the new selection will be displayed over the curves that have previously been frozen, allowing the user to compare the different data objects.

\subsubsection{Dose Depth}

The Dose Depth plot works by tracing a ray through the 3D geometry and displaying the dose data along the defined path. It also records what regions were traversed, allowing the user to see how the dose changes through the coordinate space. Creating a Dose Depth plot is similar to creating a DVH plot. Clicking the "New Plot" button on the Dose Depth panel launches the point selection panel and will ask for the starting and ending points of the ray to trace.

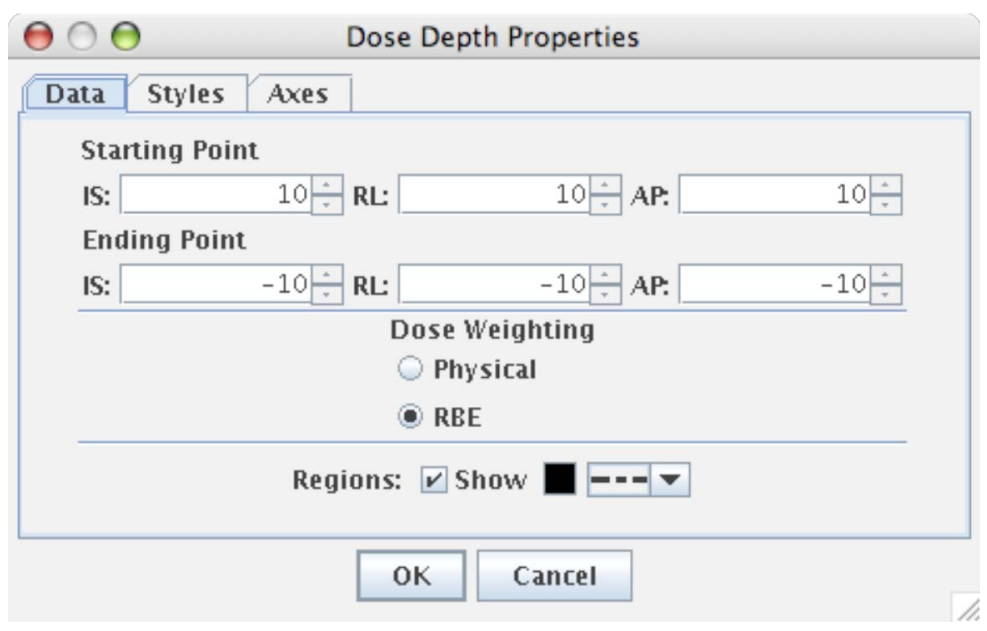

Figure 35. The dose depth plot panel.

Once the user has entered the starting and ending points, the Dose Depth plot will be displayed. In addition to the graph of the dose levels, the regions the ray traversed is displayed as a vertical line at each region boundary. 


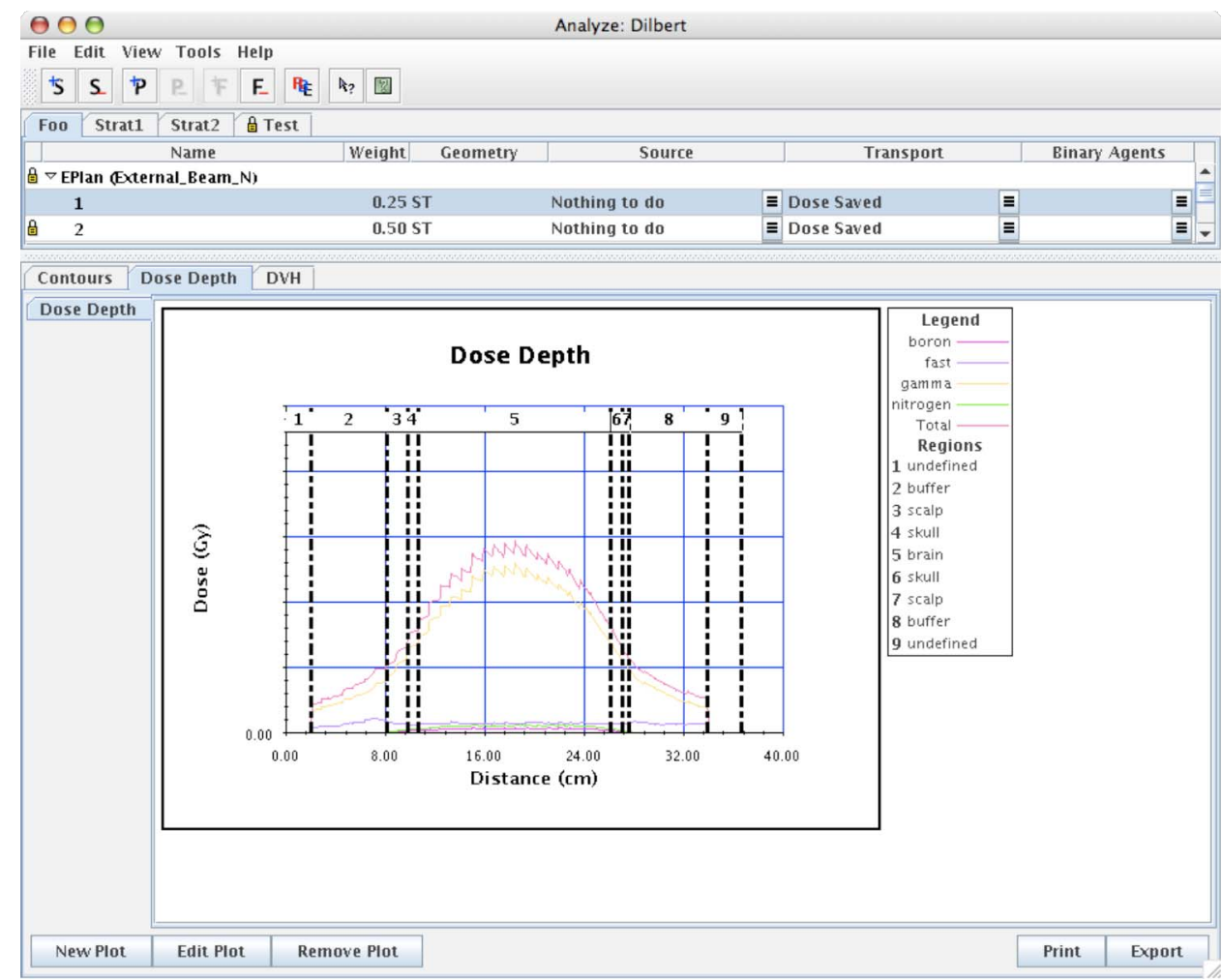

Figure 36. The dose depth plot display.

Like the other plotting engines, the user can edit the data and plot properties by clicking on the "Edit Plot" button. Printing and exporting to a graphic file is also supported. 


\section{REFERENCES}

Frandsen MW, Wessol DE, Wheeler FJ, and Starkey D, "Rapid Geometry Interrogation for Uniform Volume Element-Based BNCT Monte Carlo Particle Transport Simulation," Proceedings of the Eighth International Symposium on Neutron Capture Therapy, September 13-18, 1998, La Jolla, CA, USA, Plenum Press, New York.

Frandsen MW, Wessol DE, and Wheeler FJ, "Methods and Computer Executable Instructions for Rapidly Calculating Simulated Particle Transport Through Geometrically Modeled Treatment Volumes Having Uniform Volume Elements for Use in Radiotherapy," US Patent 6,175,761 B1, Jan 16, 2001.

Gosling J, Joy B, Steele G, and Bracha G (2005) The Java Language Specification, 3rd edition, AddisonWesley.

Viola P and Wells WM (1997) “Alignment by Maximization of Mutual Information," Int J Comp Vis 24(2) 137-154. 


\section{APPENDIX A-MYSQL SETUP}

\section{HOW TO SETUP MYSQL FOR USE WITH TIRADE}

This appendix provides minimal instructions needed to set up the MySQL database software for use with the MINERVA system. It is not a complete guide for running and administering MySQL.

The database needs to be setup on one computer at each site. After the database server is setup, each computer and MINERVA user only needs a user account on the server. To complete the installation, root access on the server system is required.

\section{Obtaining MySQL}

The MySQL database can be found at http://www.mysql.com. In the downloads section, select the latest stable production release. The next page will present you with the various MySQL system binaries. Find the appropriate system and download the "Standard" binaries.

If you choose to download Linux RPMs you will also need to get the "Client programs" package as well. Versions of MySQL before 4.1 required the "max" version. Mysql 4.1 and later include the needed features in the standard version, so the "max" version is not required.

This example will assume installation on a Linux platform using RPM. For installation on a different platform or from a tar.gz file, please see the MySQL documentation for installation and configuration instructions.

\section{Installing MySQL}

Quick install instructions:

1. su to root

2. type 'rpm -ivh MySQL-server-version.platform.rpm MySQL-client-version.platform.rpm' (using the full filename that includes the correct versions and platform )

\section{Creating the Database}

First, set a password for root to prevent unauthorized access to your database.

\section{\$/usr/bin/mysqladmin -u root password 'new-password'}

Then connect to the database

$$
\text { \$ mysql }-u \text { root }-p
$$

and create the database.

mysql> create database tirade;

\section{Adding users to MySQL}

Now add a user.

$$
\$ \text { mysql }-u \text { root }-p
$$


mysql> grant all privileges on tirade.* to username@hostname identified by 'user-password';

The hostname is the name of the computer used to run MINERVA, not the server name.

This can be repeated for any number of users by changing the hostname, username, and password. After all users have been added, type 'quit' to exit the mysql shell.

\section{Creating the MySQL configuration file}

The MySQL configuration file will be run when the MySQL server starts. Several tiers of my.cnf files can be setup, but for simplicity only the server specific file will be setup. If installing via RPM (as shown above), create the file /var/lib/mysq1/my.cnf. If installing MySQL by a different method or in a different location, or to learn more about the my.cnf file, please see the MySQL documentation for more details.

As root, open the my.cnf file in the specified location (it may be necessary to create the file if this is the first time anything has been added to it). There are two lines that must be added for MINERVA. A minimal configuration file should look like:

[mysqld]

max_allowed_packet $=8 \mathrm{M}$

The first line lets MySQL know that this is a valid configuration file. The second line sets the max allowed packet size to $8 \mathrm{MB}$. This is needed because of the large binary objects will be stored in the database. After saving this file, perform the following commands as root:

\# letc/init.d/mysql restart

\# mysqladmin variables | grep max_allowed_packet

The second command should show the value for max_allowed_packet, which should output something like:

max_allowed_packet | 8387584

Note: If the max_allowed_packet is not set correctly, MINERVA will likely fail when saving binary data. $8 \mathrm{MB}$ is not necessarily the smallest packet size possible, but during testing it has consistently worked.

The new MySQL database is ready for use with MINERVA. For additional information on database security, please see the MySQL documentation. 


\section{APPENDIX B-POSTGRESQL SETUP}

\section{HOW TO SETUP POSTGRESQL FOR USE WITH TIRADE}

This appendix provides minimal instructions needed to set up the PostgreSQL database software for use with the MINERVA system. It is not a complete guide for running and administering PostgreSQL.

The database needs to be setup on one computer at each site. After the database server is setup, each computer and user that uses MINERVA only needs a user account on the server. To complete the installation, database admin access on the server system is required.

\section{Obtaining PostgreSQL}

The PostgreSQL database can be found at http://www.postgresql.org. In the downloads section, select the latest stable production release. Most Linux and BSD distributions have PostgreSQL available in there package management system. For Fedora, the command:

yum install postgresql-server

will install the PostgreSQL server on your computer.

\section{Post install of PostgreSQL}

Quick setup instructions:

1. su to root

2. Start the postgreSQL service. Either a graphical service starting program such as serviceconf or the command line tools service and chkconfig can be used.

\section{Creating the Minerva Database}

Switch to the postgres user (or another use that has administrative privileges for the database)

\# su postgres

Then connect to the database

\$psql

and create the database.

postgres $=\#$ create database minerva;

\section{Adding users to PostgreSQL}

Now add a user.

$$
\begin{aligned}
& \text { \# su postgres } \\
& \text { \$psql } \\
& \text { postgres=\# create user fred with password 'password'; } \\
& \text { postgres }=\# \text { create database fred; }
\end{aligned}
$$

Note that with PostgreSQL and Minerva, a database with the user's name must be created, otherwise Minerva will not be able to connect. 


\section{Allowing Remote access to the Database}

By default on Fedora PostgreSQL is not set up to allow remote access. First, the firewall needs to let port 5432 through. Then in the directory $/ \mathrm{var} / \mathrm{lib} / \mathrm{pgsq} 1 /$ data the following configuration files need to be configured. The pg_hba.conf file should have an entry to allow the user to connect from the remote file add a following line (with correct username and the ip address of the computer that they are connecting from substituted) for each user and computer that will connect:
host all
fred
192.168 .123 .45
ma5

The postgresql.conf needs to allow remote connections so add or edit the following lines:

listen addresses $=1 *$ '

port $=5432$

This will allow the database to be accessed from a remote computer. 


\section{APPENDIX C-ADDITIONAL PACKAGES FOR INSTALLATION}

\section{ADDITIONAL PACKAGES NEEDED FOR MINERVA INSTALLATION}

In addition to the database software, several Java packages are necessary to properly install the MINERVA software system. All are available as free, publicly-distributed software packages; however, several have license restrictions on redistribution. Thus, each MINERVA user site will need to assure that these packages are installed on the local hardware before installing MINERVA. The following is a complete list of the necessary packages, along with the URLs to download each package:

JOGL https://jogl.dev.java.net/ The July 12, 20051.1 .1 version is required. More recent JOGL versions will not work.

JAI $\quad$ http://java.sun.com/products/java-media/jai/index.jsp

$\mathrm{dcm} 4 \mathrm{che} \quad \underline{\mathrm{http}} / / /$ sourceforge.net/projects/dcm4che/ (Included in installation)

JDOM $\quad$ http://www.jdom.org (Included in installation)

JavaHelp $\quad \underline{\mathrm{http}: / / j a v a . s u n . c o m / p r o d u c t s / j a v a h e l p / d o w n l o a d ~ b i n a r y . h t m l ~(I n c l u d e d ~ i n ~ i n s t a l l a t i o n) ~}$

JUNIT $\quad$ http://www.junit.org/index.htm Only required for compiling program.

$\log 4 \mathrm{j} \quad$ http://logging.apache.org/log $4 \mathrm{j} / \mathrm{docs} /$ (Included in installation)

Vecmath $\quad \underline{\text { http://java.freehep.org/jcvslet/JCVSlet/log/freehep/freehep/tools/vecmath.jar/0 }}$ (Included in installation)

Xerces $\quad$ http://xerces.apache.org/xerces-j/ (Included in installation)

In addition, the JDBC connector for the database will be required. (PostgreSQL and Cloudscape connectors are included in installation.) 


\section{APPENDIX D-XML FILE WITH DOSESET}

\section{EXAMPLE XML FILE WITH DOSESET}

his is an example xml file of a test patient that could be used as a guide for constructing the API between MINERVA and the transport plug-in. Elements with a bin extension would be provided by the exporter.

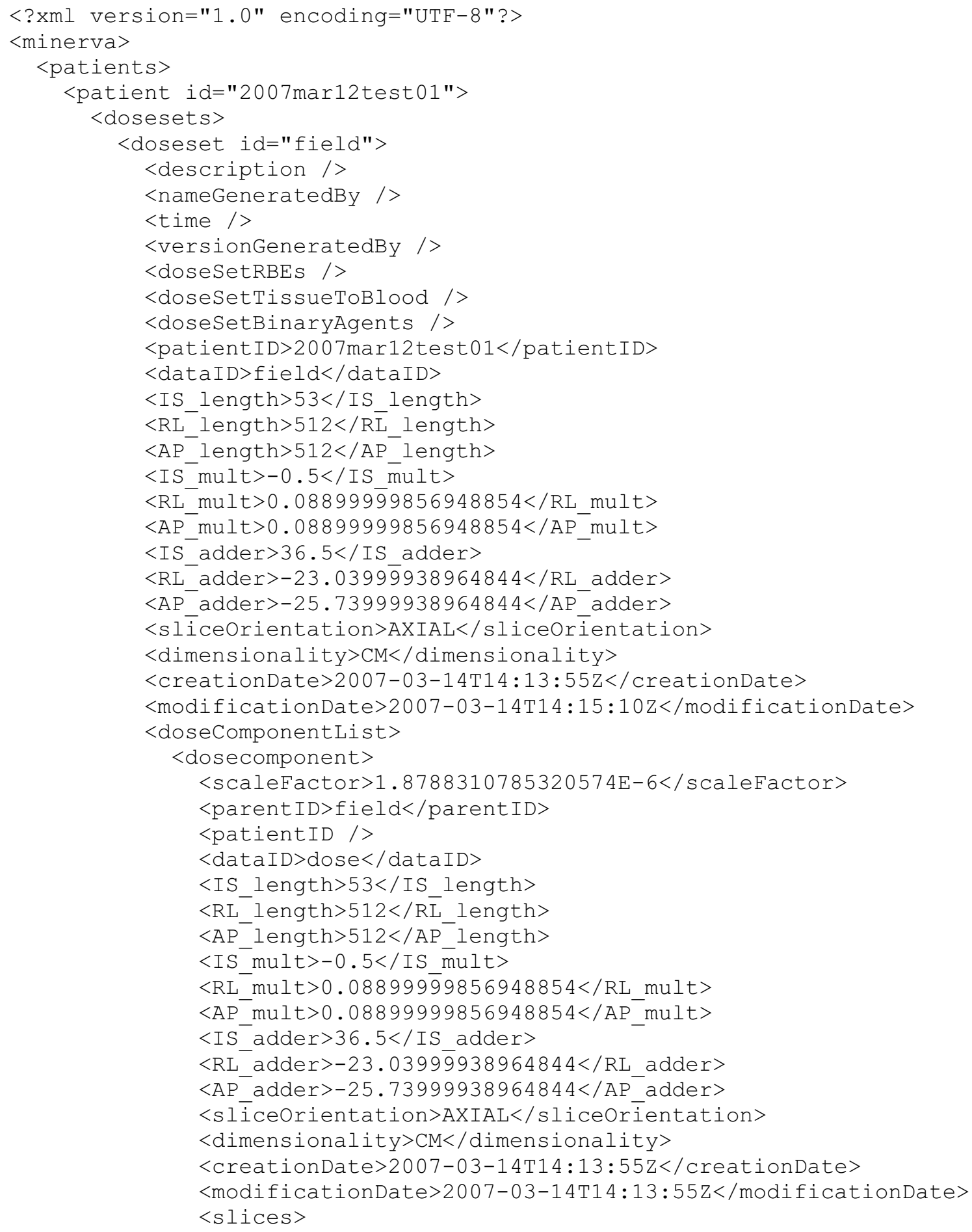




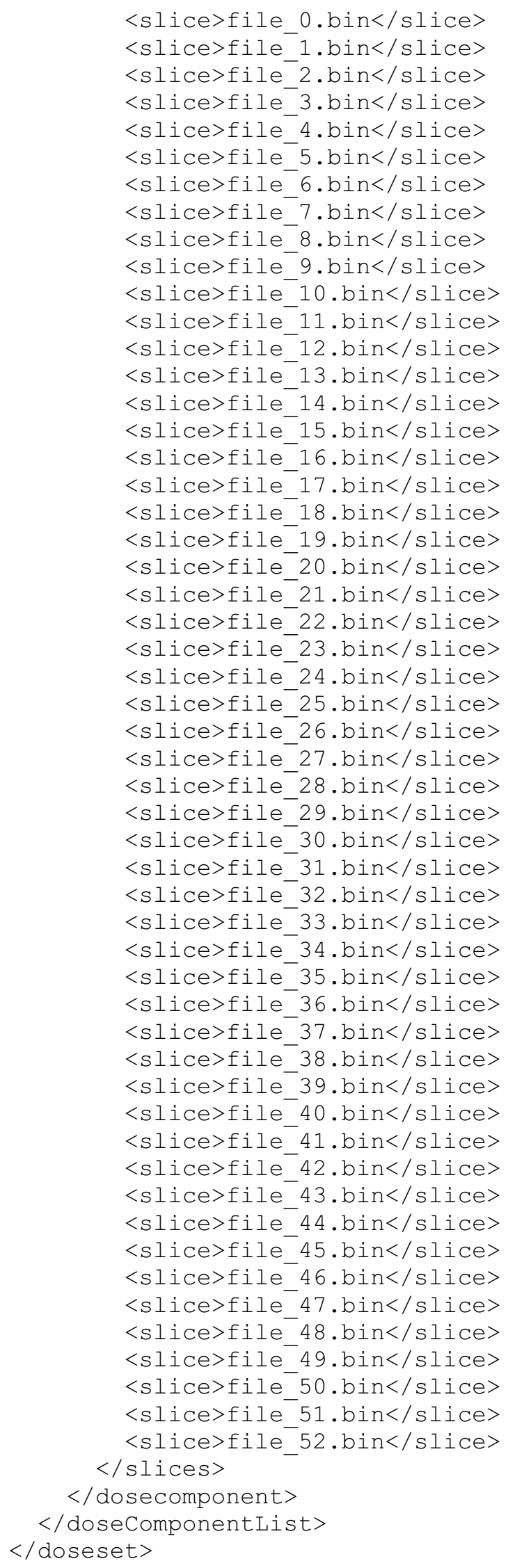

Appendix D-ii 


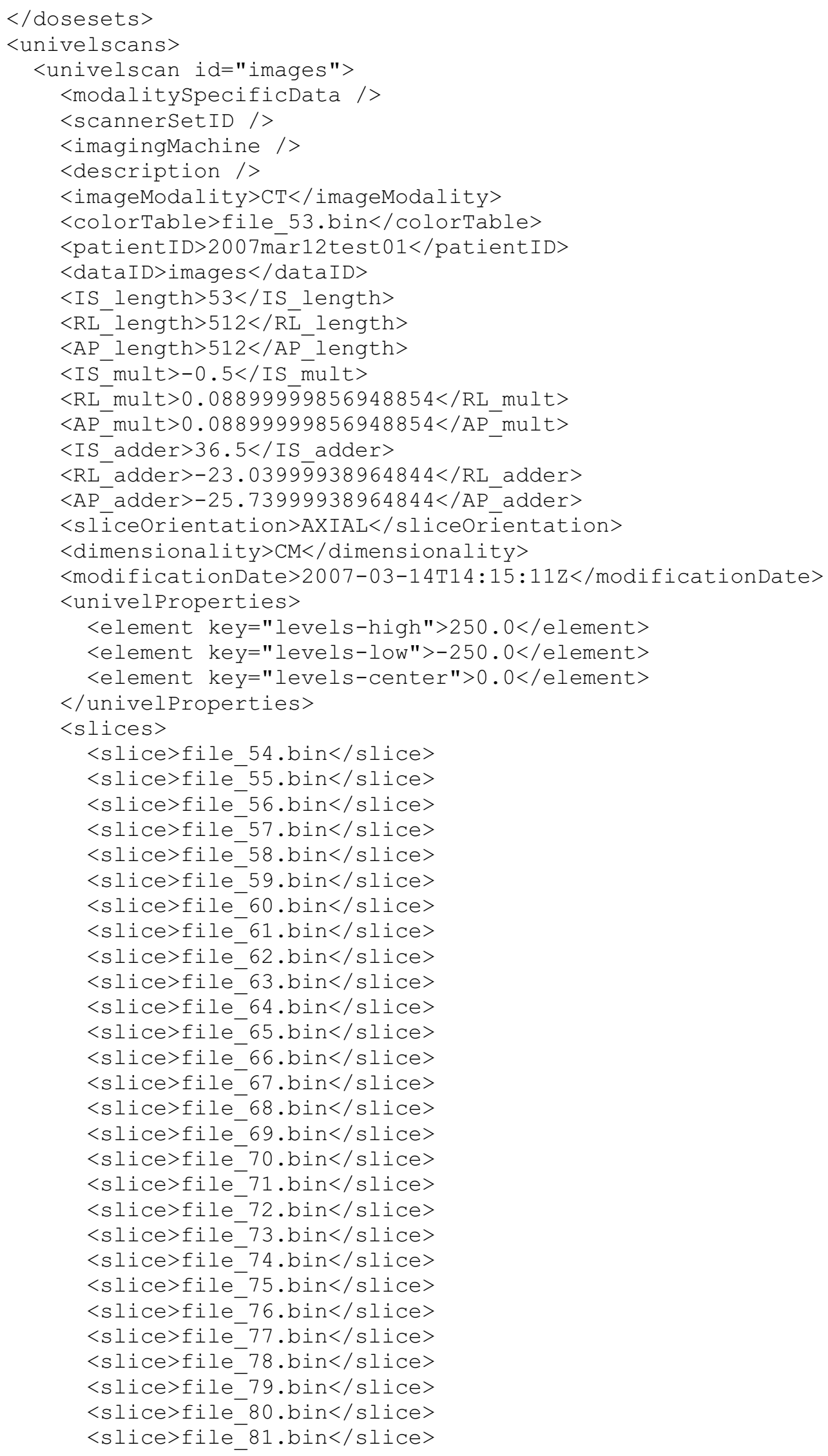

\section{Appendix D-iii}




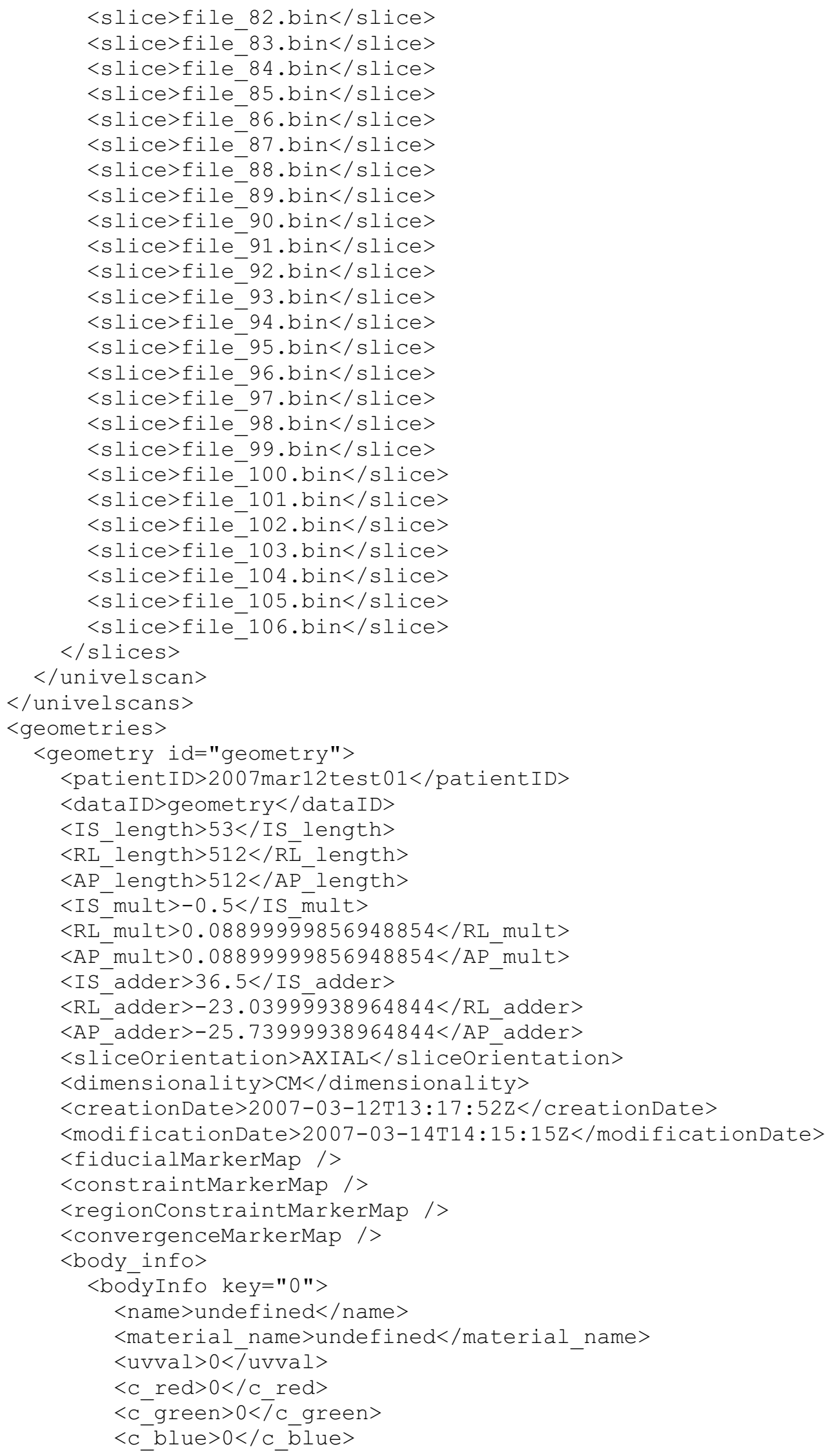




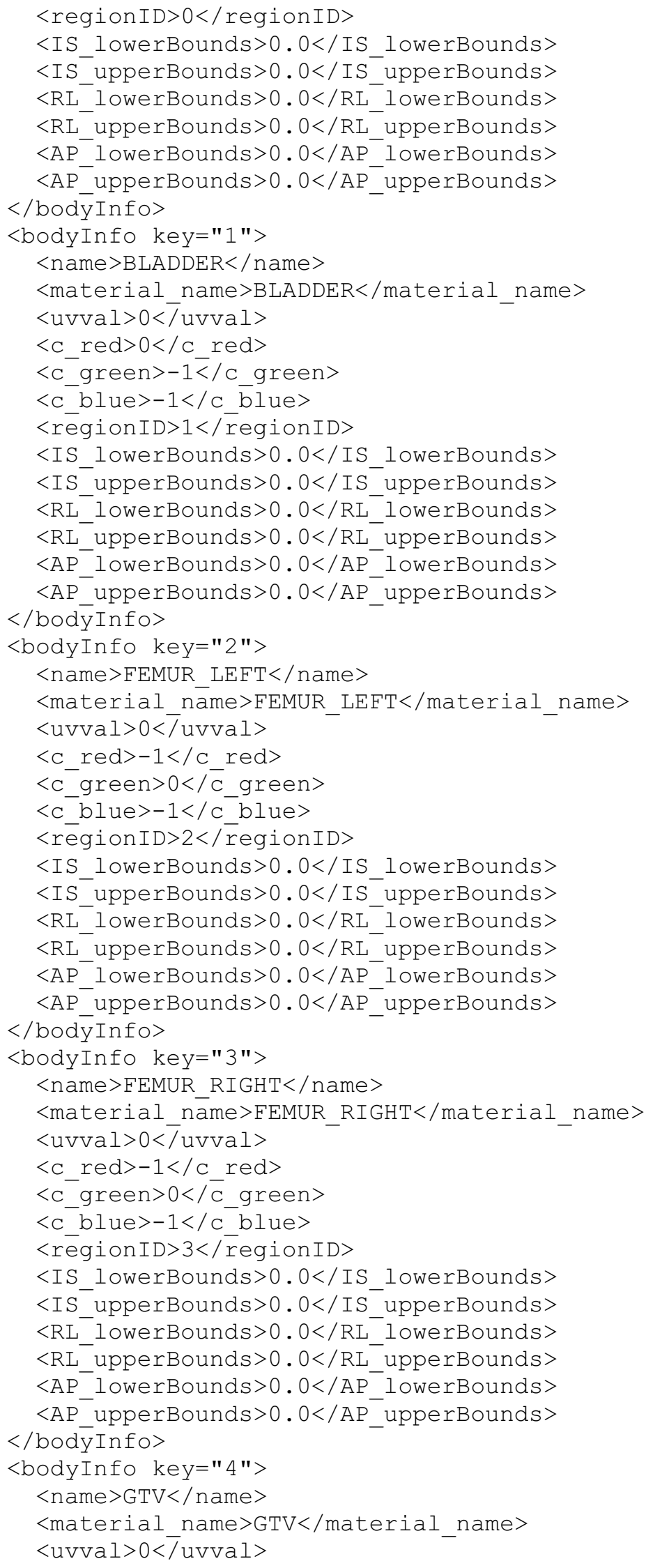

\section{Appendix D-V}




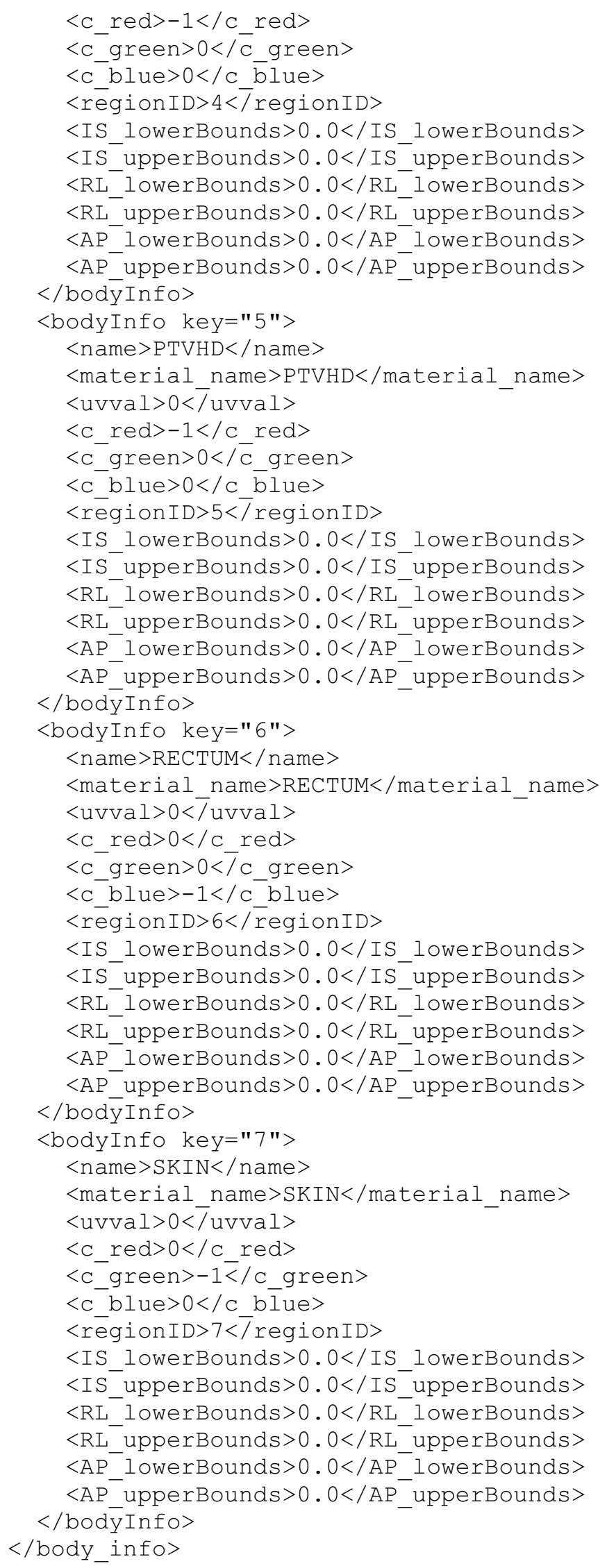

\section{Appendix D-vi}




$$
\begin{aligned}
& <\text { imagesets }> \\
& \text { <element>images }</ \text { element }> \\
& </ \text { imagesets }> \\
& <\text { materialHash / > } \\
& <\text { slices }> \\
& \text { <slice>file_107.bin</slice }> \\
& \text { <slice>file 108.bin</slice }> \\
& \text { <slice>file } 109 . \text { bin</slice }> \\
& \text { <slice>file_110.bin</slice }> \\
& \text { <slice>file 111.bin</slice }> \\
& \text { <slice>file_112.bin</slice }> \\
& \text { <slice>file_113.bin</slice }> \\
& \text { <slice>file } 114 \text {.bin</slice }> \\
& \text { <slice>file-115.bin</slice }> \\
& \text { <slice>file_116.bin</slice }> \\
& \langle\text { slice }>\text { file_117.bin }</ \text { slice }> \\
& \text { <slice>file-118.bin</slice }> \\
& \text { <slice>file } 119 . \text { bin</slice }> \\
& \text { <slice>file-120.bin</slice }> \\
& \text { <slice>file_121.bin</slice }> \\
& \text { <slice>file_122.bin</slice }> \\
& \text { <slice>file_123.bin</slice }> \\
& \text { <slice>file-124.bin</slice }> \\
& \text { <slice>file } 125 \text {.bin</slice }> \\
& \text { <slice>file_126.bin</slice }> \\
& \text { <slice>file_127.bin</slice }> \\
& <\text { slice }>\text { file_128.bin</slice }> \\
& <\text { slice }>\text { file } 129 . b i n</ \text { slice }> \\
& \text { <slice>file } 130 . \text { bin</slice }> \\
& \text { <slice>file_131.bin</slice }> \\
& \text { <slice>file_132.bin</slice }> \\
& \text { <slice }>\text { file_133.bin</slice }> \\
& \text { <slice>file-134.bin</slice }> \\
& \text { <slice>file } 135 \text {.bin</slice }> \\
& \text { <slice>file-136.bin</slice }> \\
& \text { <slice>file_137.bin</slice }> \\
& \text { <slice>file_138.bin</slice }> \\
& \text { <slice>file_139.bin</slice }> \\
& \text { <slice>file-140.bin</slice }> \\
& \text { <slice>file 141.bin</slice }> \\
& \text { <slice>file-142.bin</slice }> \\
& \text { <slice>file_143.bin</slice }> \\
& \text { <slice>file_144.bin</slice }> \\
& \text { <slice>file } 145 \text {.bin</slice }> \\
& \text { <slice>file } 146 . b i n</ \text { slice }> \\
& \text { <slice>file-147.bin</slice }> \\
& \text { <slice>file_148.bin</slice }> \\
& \text { <slice>file_149.bin</slice }> \\
& \text { <slice>file-150.bin</slice }> \\
& \text { <slice }>\text { file } 151 \text {.bin</slice }> \\
& \text { <slice>file_152.bin</slice }> \\
& \text { <slice>file_153.bin</slice }> \\
& \text { <slice>file_154.bin</slice }> \\
& \text { <slice>file_155.bin</slice }> \\
& \text { <slice>file-156.bin</slice }> \\
& \text { <slice>file-157.bin</slice }> \\
& \text { <slice>file_158.bin</slice }>
\end{aligned}
$$

Appendix D-vii 


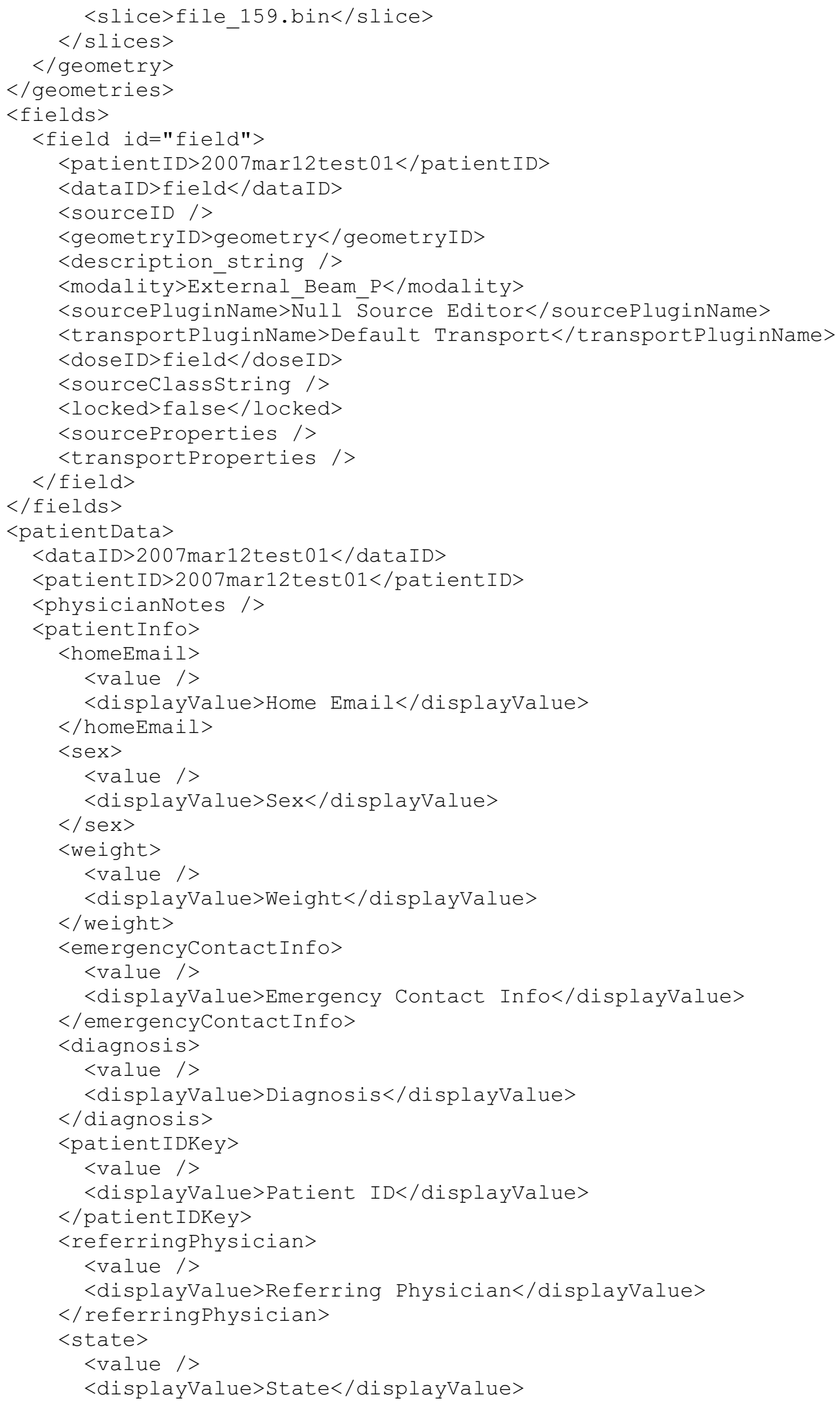

\section{Appendix D-viii}




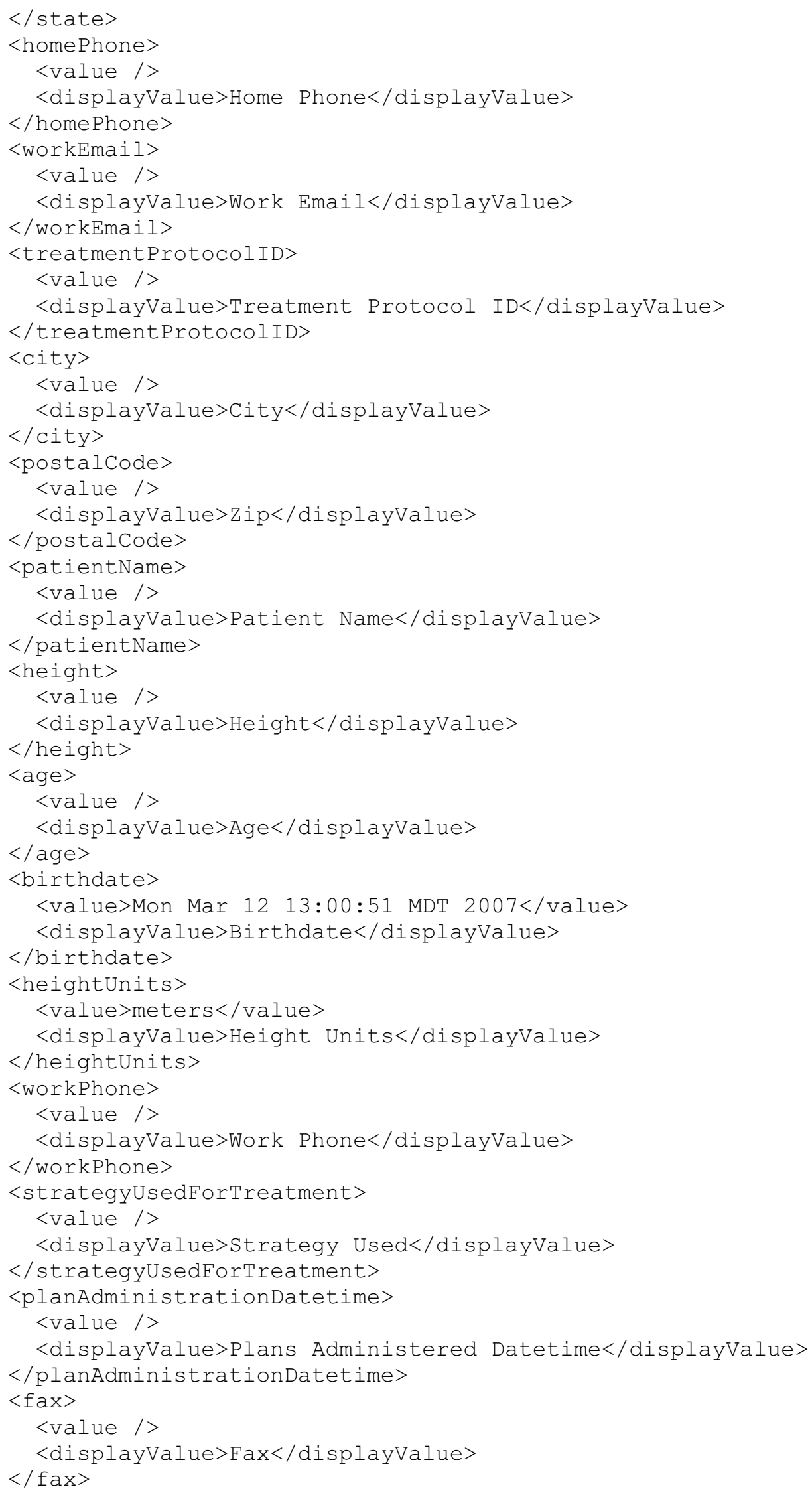

\section{Appendix D-ix}




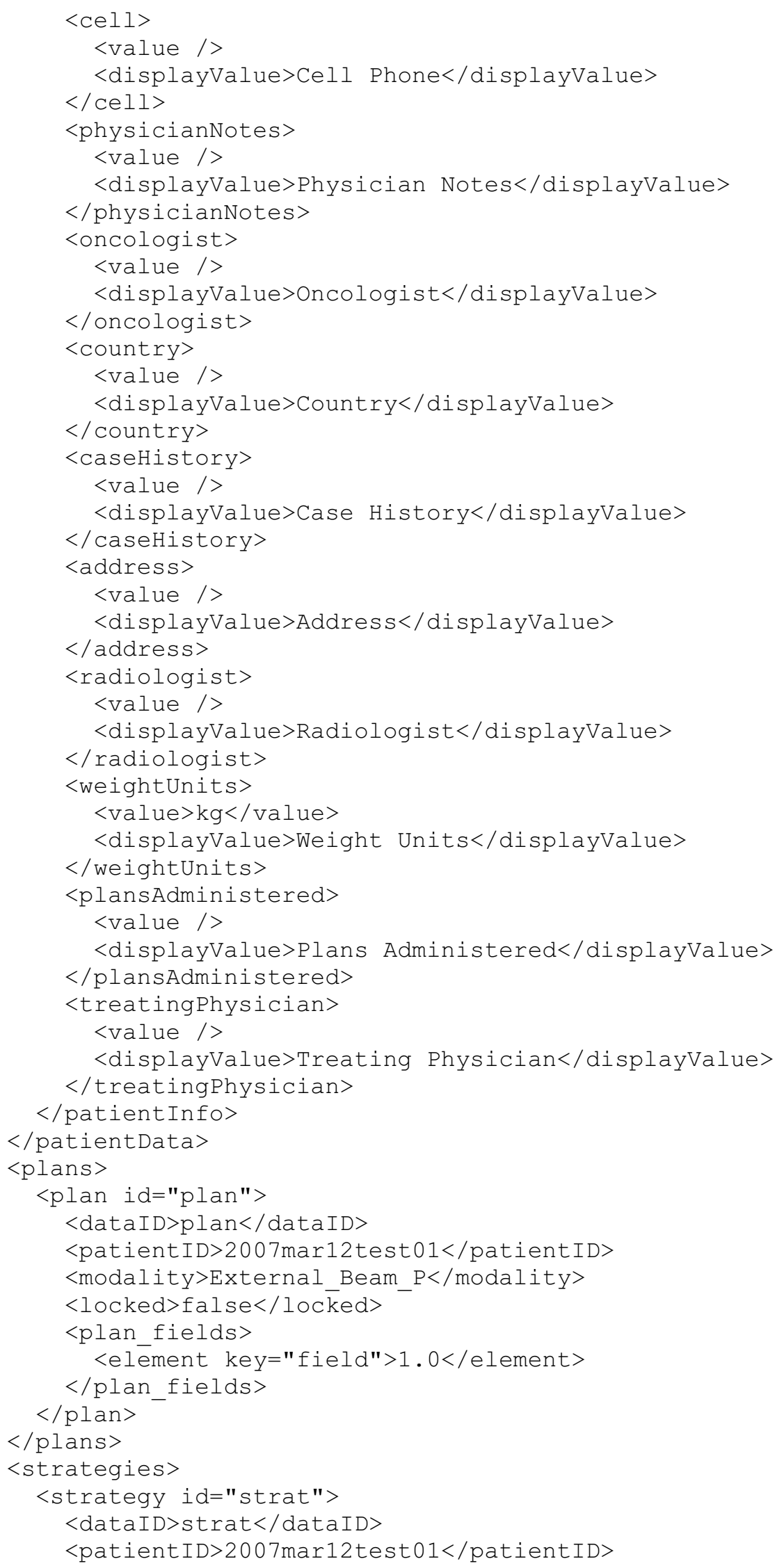

\section{Appendix D-X}




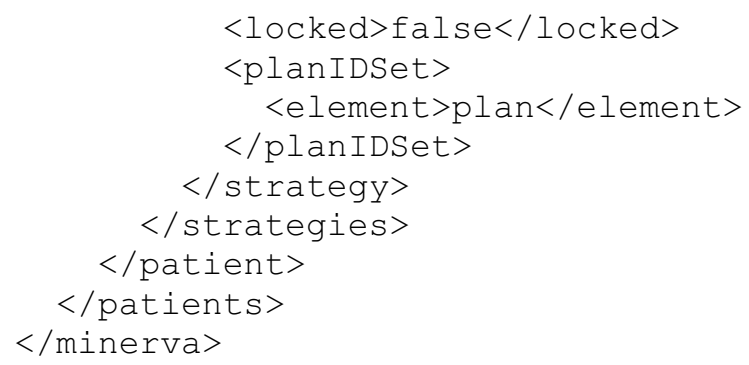




\section{APPENDIX E-XML FILE WITH SOURCE}

EXAMPLE XML FILE WITH SOURCE

This is an example xml file of a test patient that could be used as a guide for constructing the API between MINERVA and the source plugin. Elements with a bin extension would be provided by the exporter.

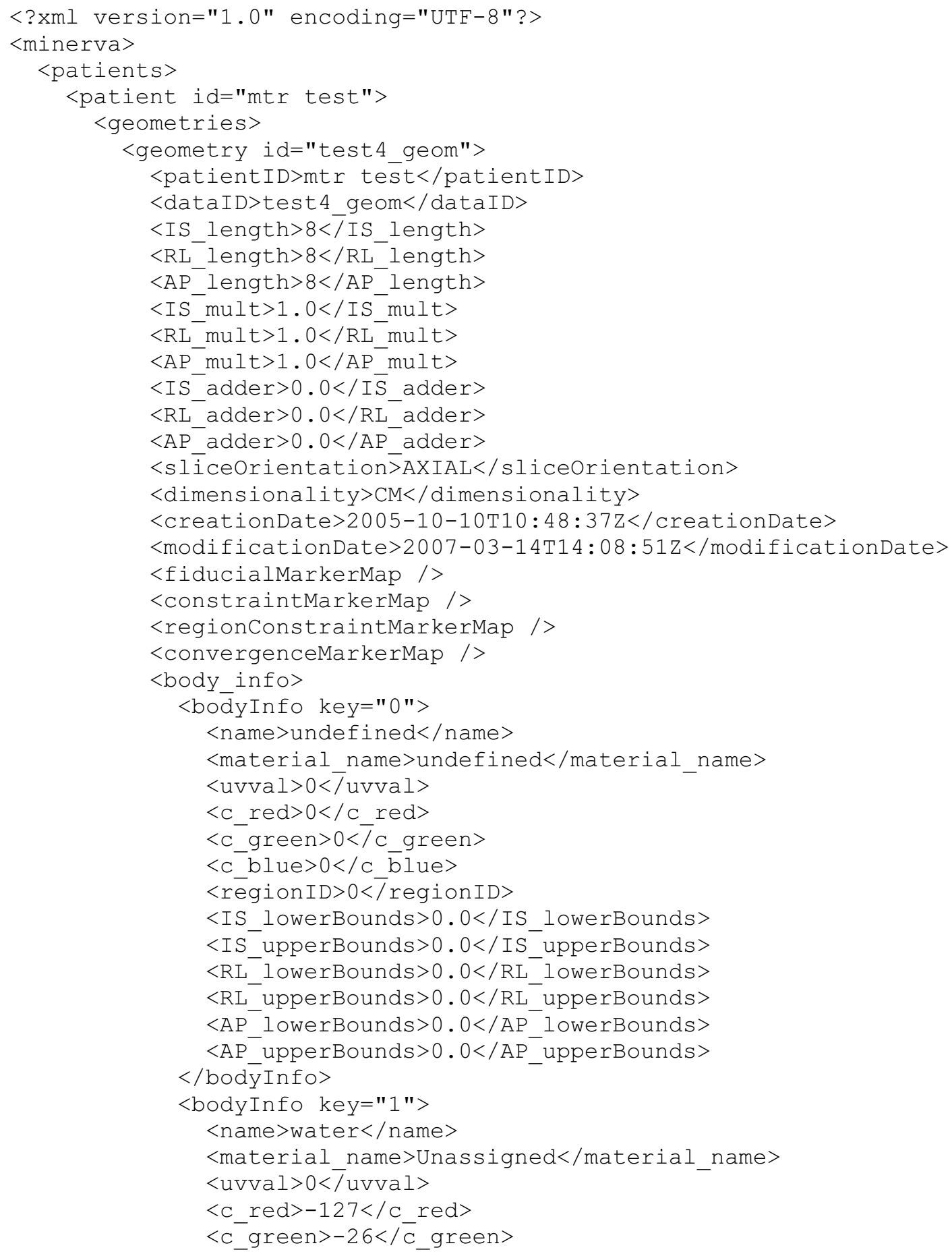




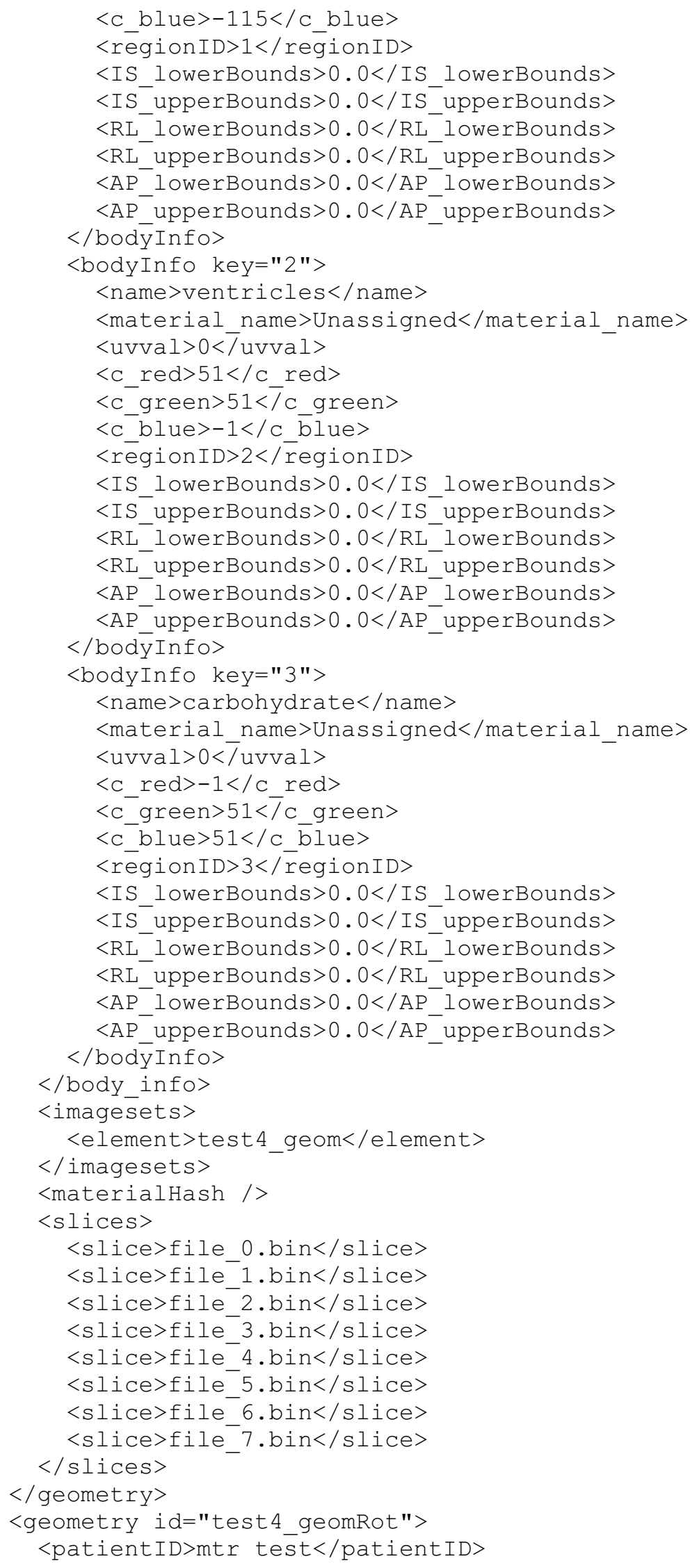

Appendix E-ii 


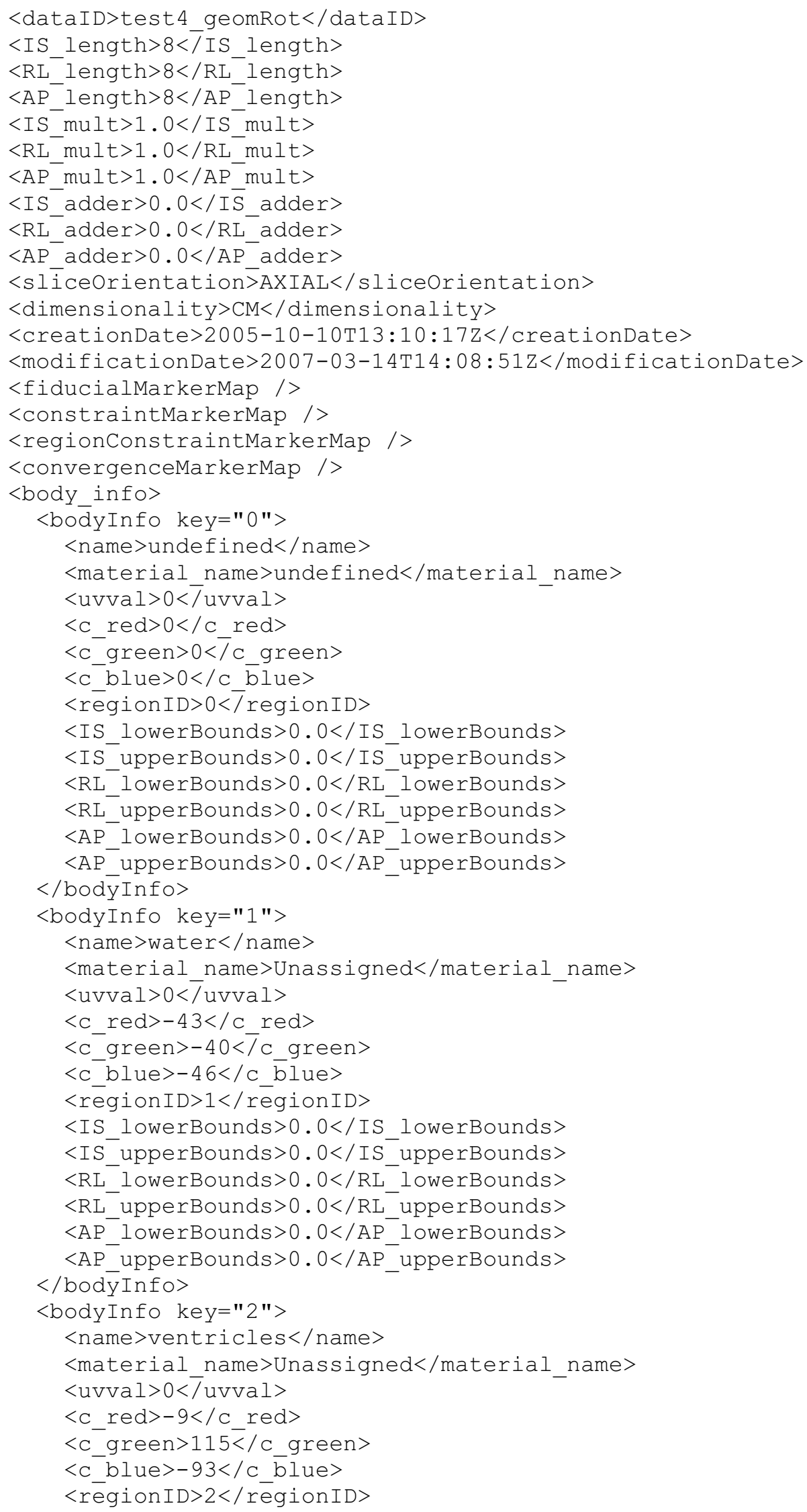

\section{Appendix E-iii}




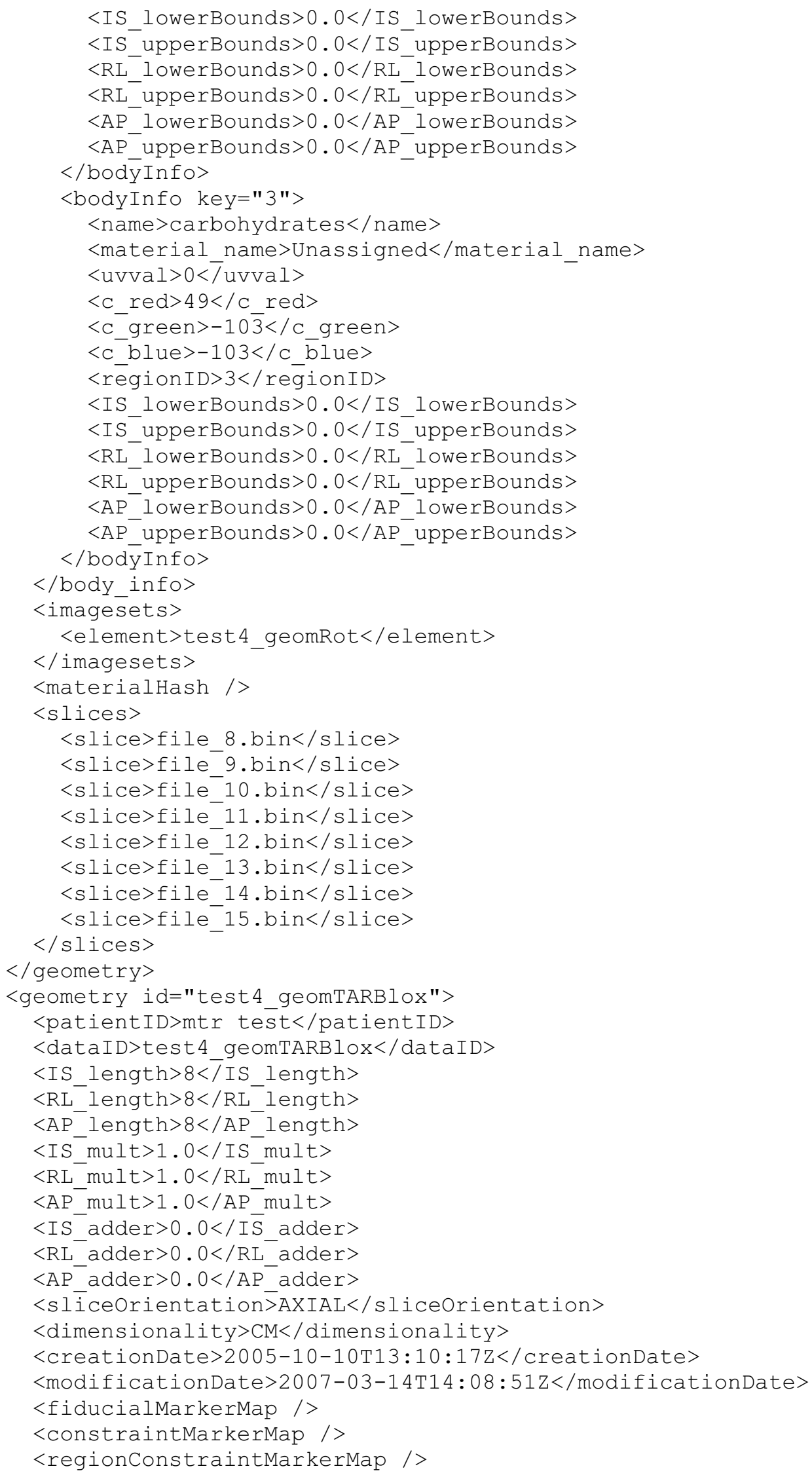

\section{Appendix E-iv}




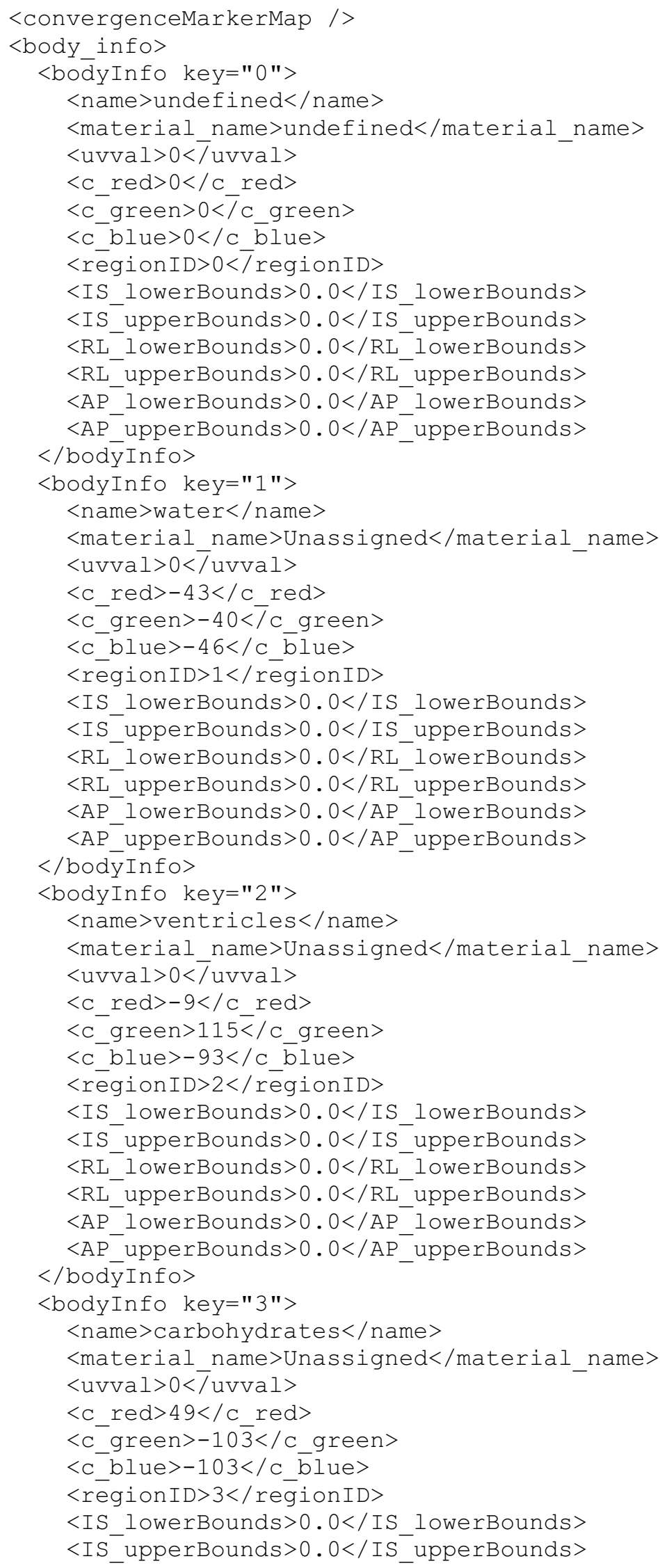




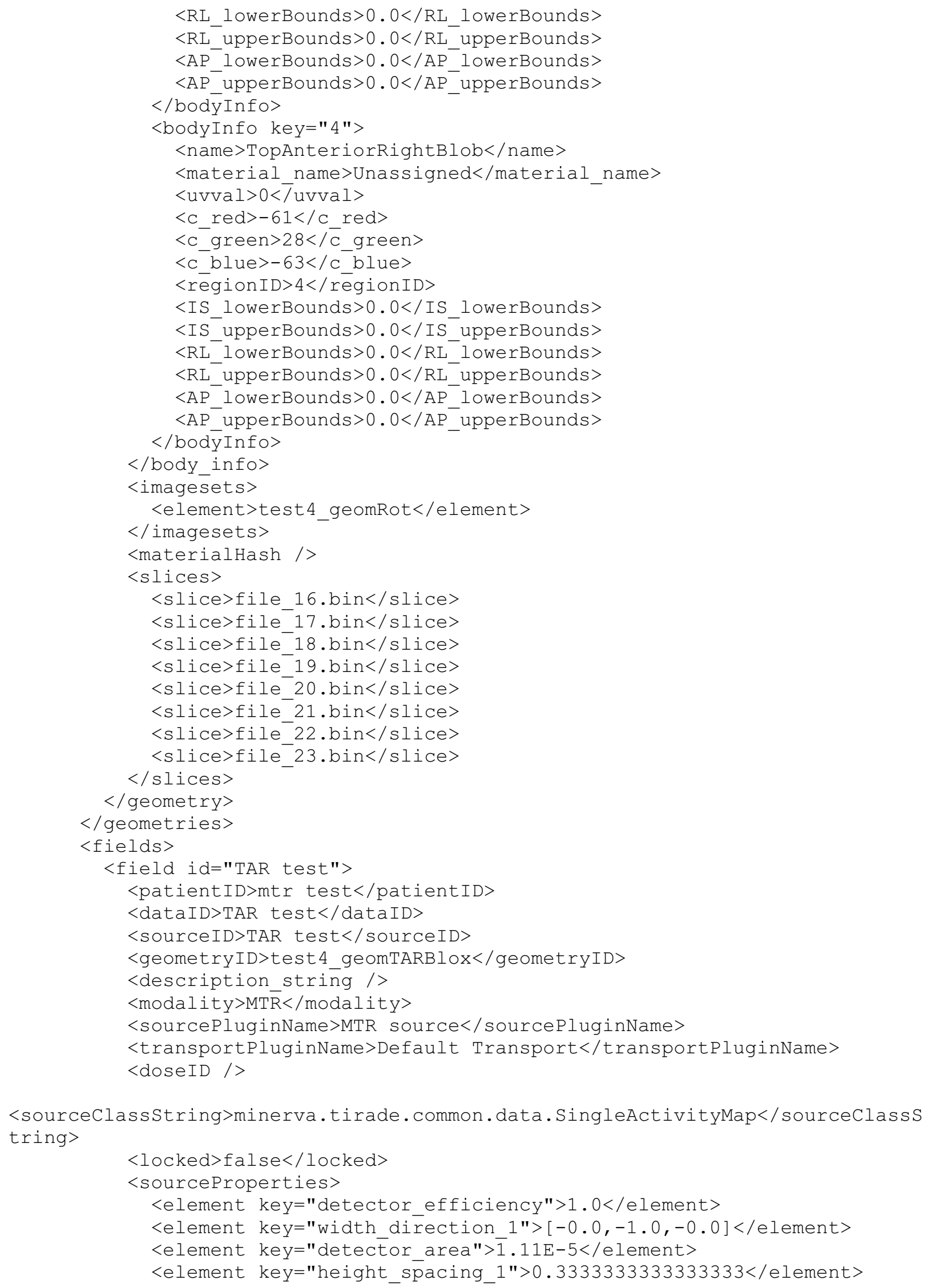

\section{Appendix E-vi}




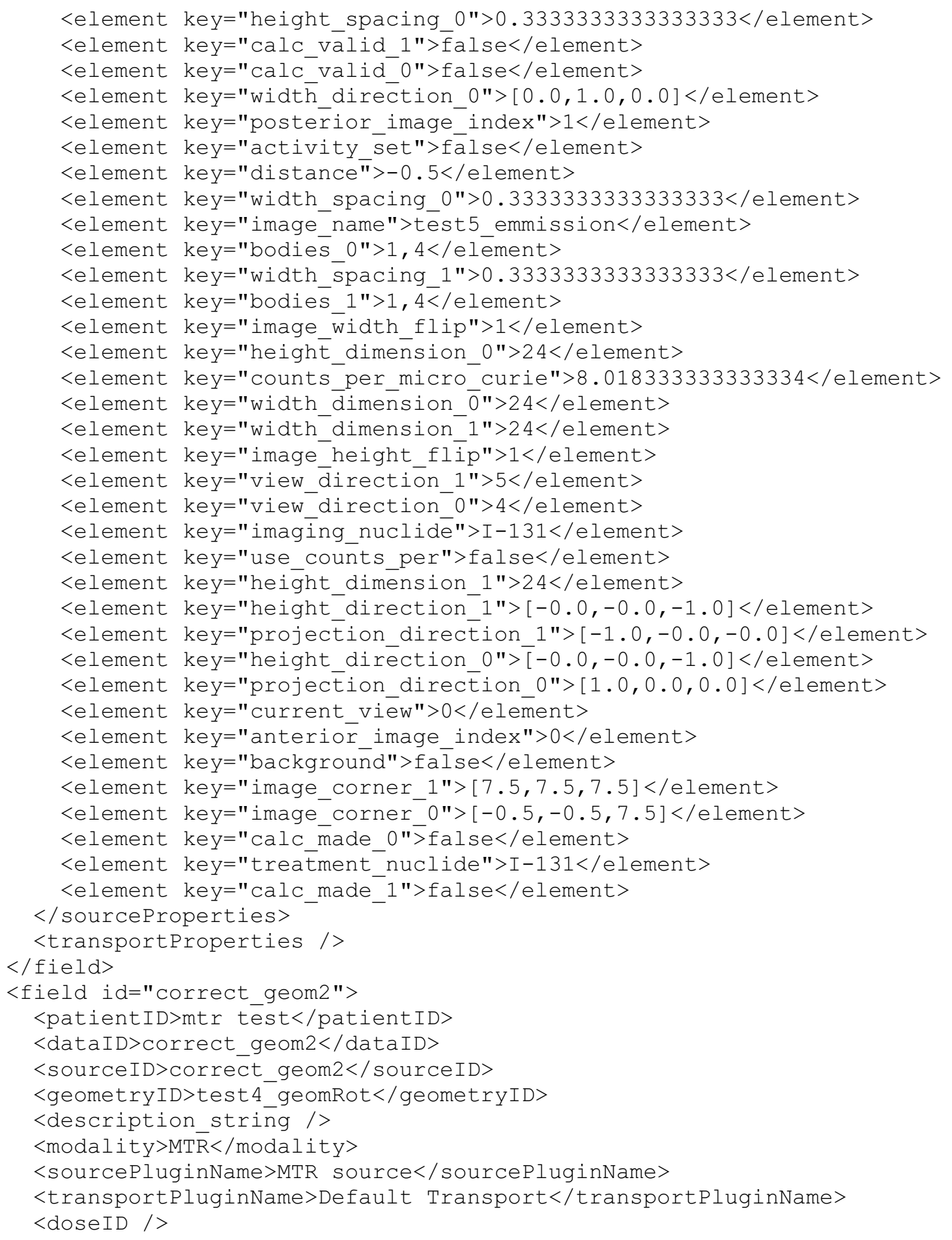

<sourceClass.String>minerva.tirade. common.data. SingleActivityMap</sourceClass. tring $>$

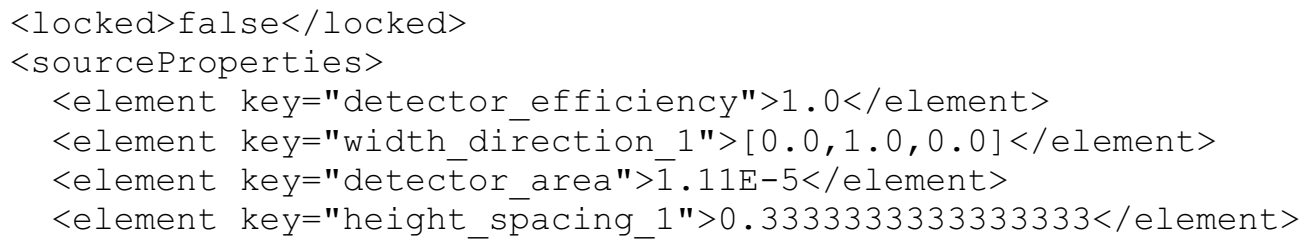




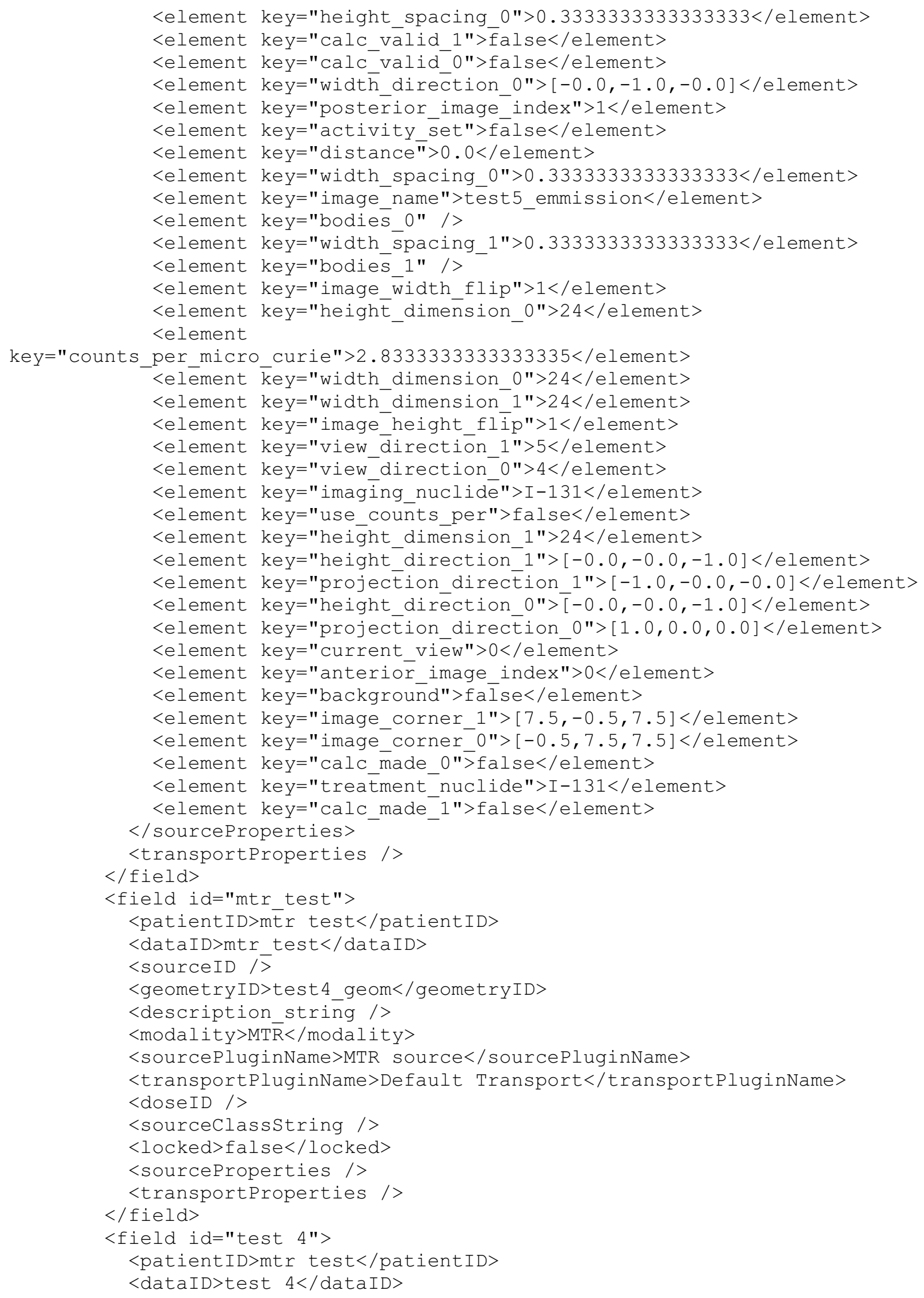

\section{Appendix E-viii}




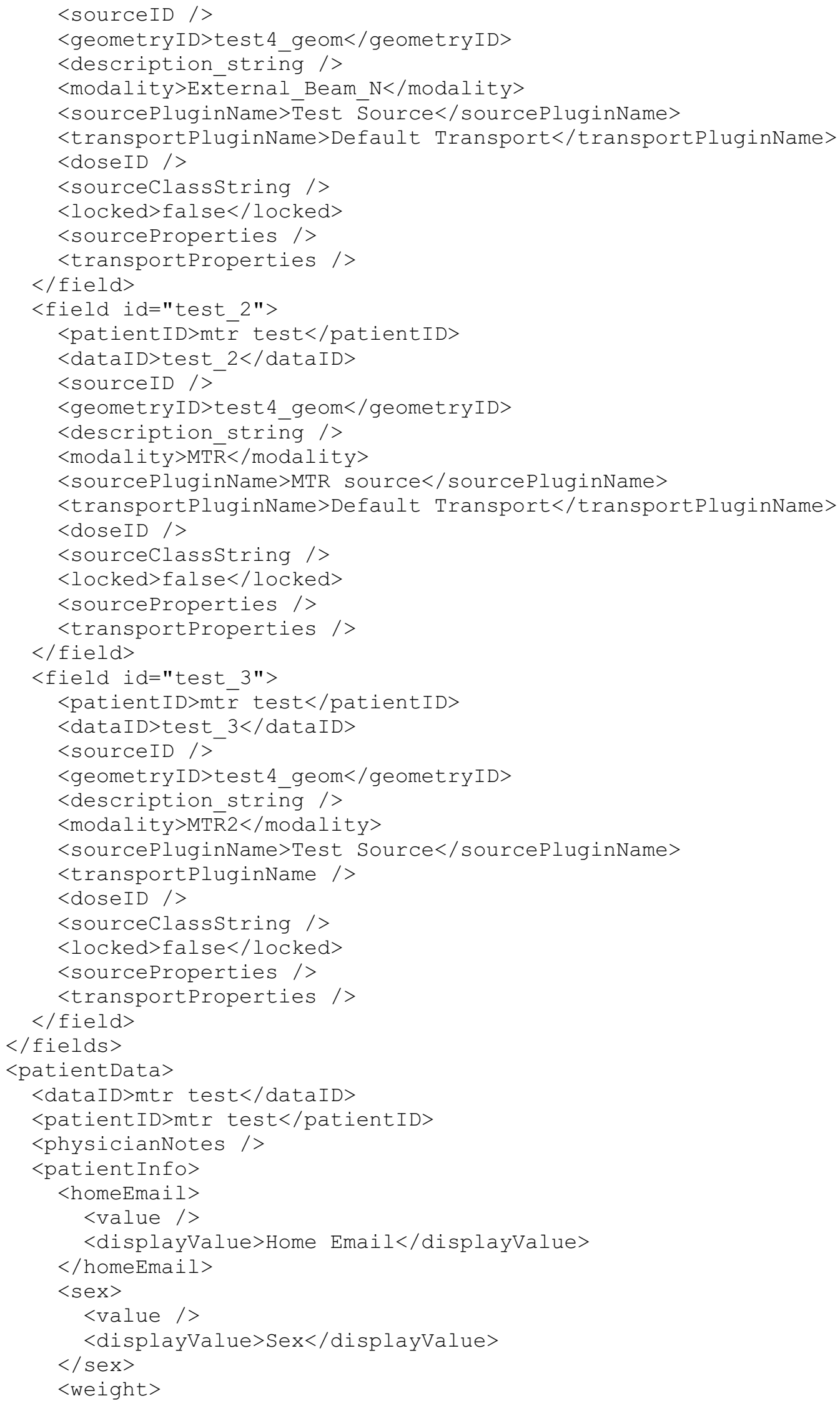

\section{Appendix E-ix}




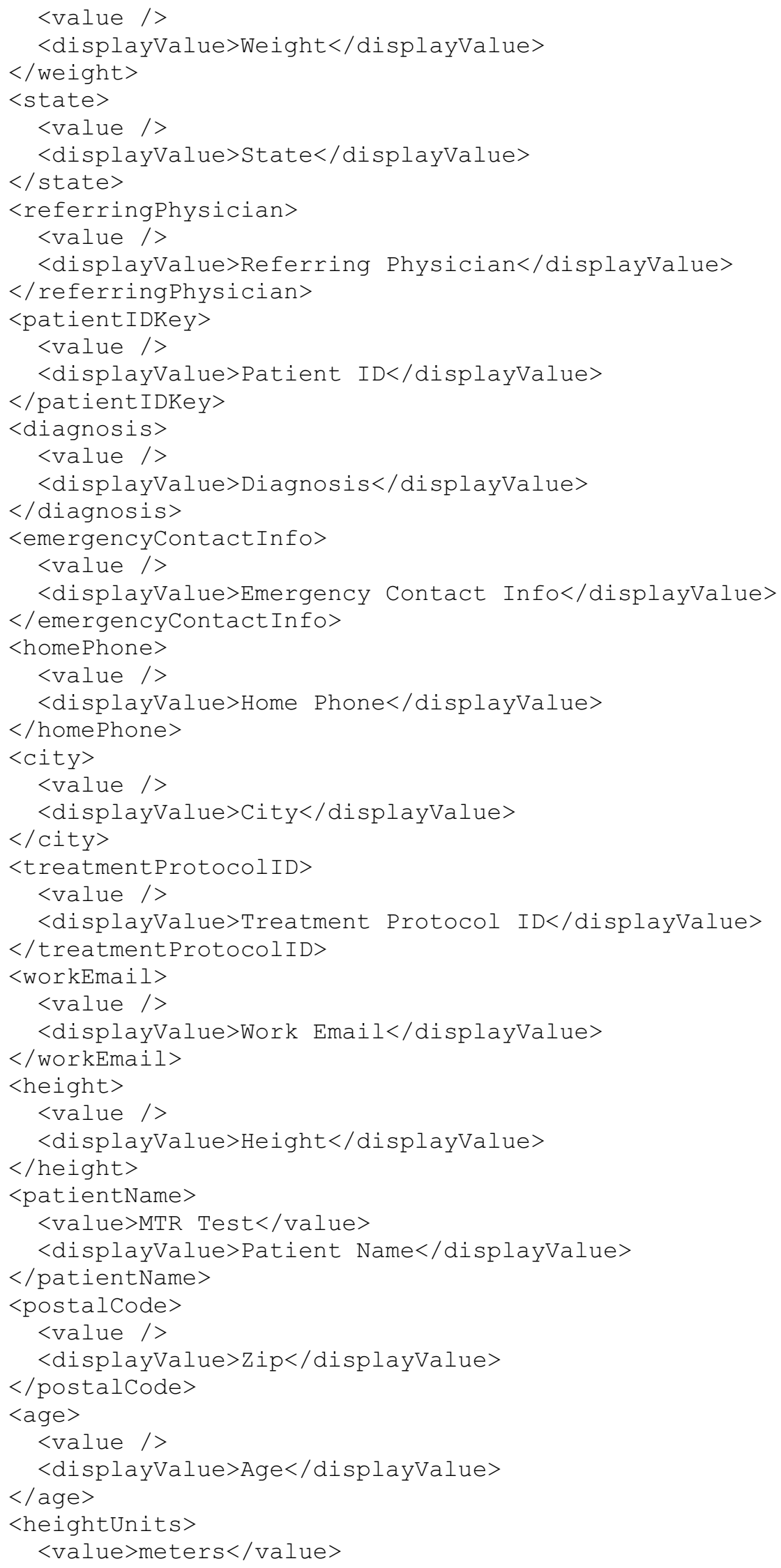

Appendix E-X 


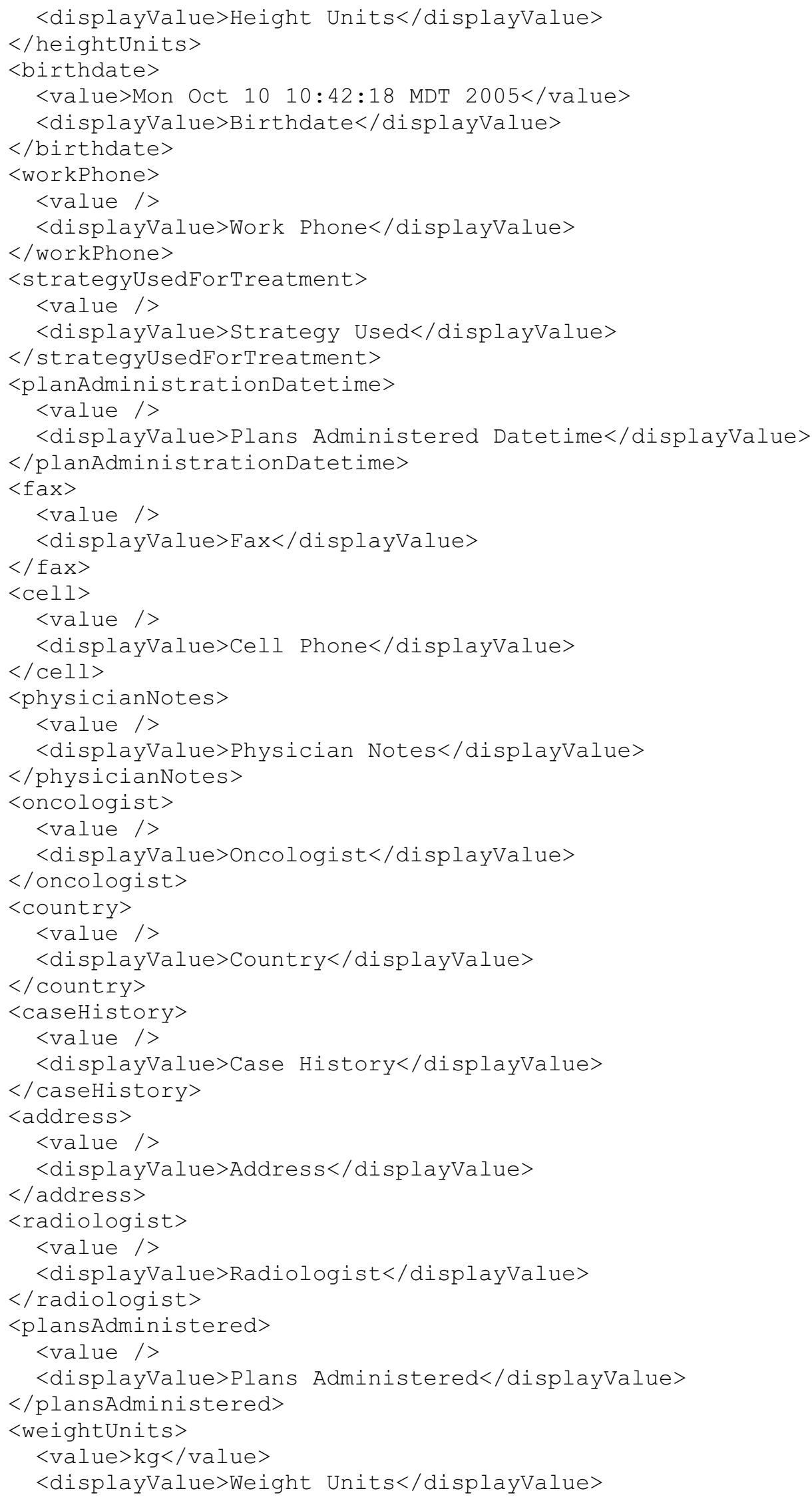

\section{Appendix E-xi}




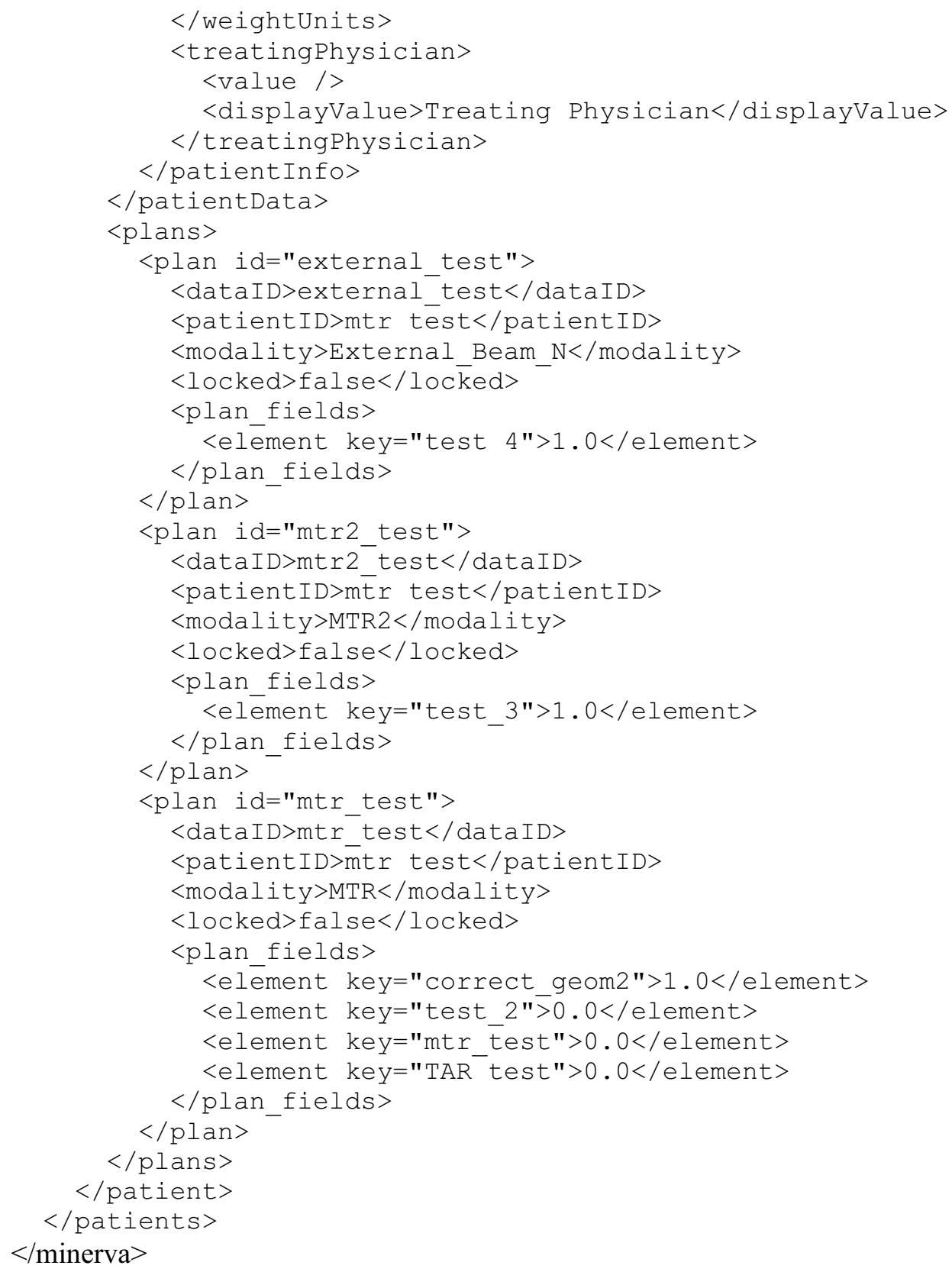

